\title{
BRAZILIAN SOCIETY OF HEPATOLOGY RECOMMENDATIONS FOR THE DIAGNOSIS AND MANAGEMENT OF AUTOIMMUNE DISEASES OF THE LIVER
}

\author{
Paulo Lisboa BITTENCOURT ${ }^{1}$, Eduardo Luiz Rachid CANÇADO², Cláudia Alves COUTO ${ }^{3}$, \\ Cynthia LEVY ${ }^{4}$, Gilda PORTA ${ }^{2}$, Antônio Eduardo Benedito SILVA ${ }^{5}$, \\ Debora Raquel Benedita TERRABUIO ${ }^{2}$ and Members of the Pannel of the 1st Consensus of \\ the Brazilian Society of Hepatology on the Diagnosis and Management of Autoimmune Diseases \\ of the Liver*
}

\begin{abstract}
In order to draw evidence-based recommendations concerning the management of autoimmune diseases of the liver, the Brazilian Society of Hepatology has sponsored a single-topic meeting in October 18th, 2014 at São Paulo. An organizing committee comprised of seven investigators was previously elected by the Governing Board to organize the scientific agenda as well as to select twenty panelists to make a systematic review of the literature and to present topics related to the diagnosis and treatment of autoimmune hepatitis, primary sclerosing cholangitis, primary biliary cirrhosis and their overlap syndromes. After the meeting, all panelists gathered together for the discussion of the topics and the elaboration of those recommendations. The text was subsequently submitted for suggestions and approval of all members of the Brazilian Society of Hepatology through its homepage. The present paper is the final version of the reviewed manuscript organized in topics, followed by the recommendations of the Brazilian Society of Hepatology.
\end{abstract}

HEADINGS - Autoimmune hepatitis. Primary sclerosing cholangitis. Primary biliary cirrhosis. Diagnosis. Treatment.

\section{INTRODUCTION}

The Brazilian Society of Hepatology has sponsored in October $18^{\text {th }}, 2014$ at São Paulo, the first meeting concerning the management of autoimmune liver diseases (ALD), in order to draw evidence-based recommendations concerning the diagnosis and treatment of autoimmune hepatitis (AIH), primary sclerosing cholangitis (PSC), primary biliary cirrhosis (PBC) and their overlap syndromes. The governing board of the Brazilian Society of Hepatology elected seven investigators with recognized expertise and/or publications in the field to organize the scientific agenda. They have selected the topics to be reviewed, including the diagnosis and treatment of AIH, PSC, PBC and overlap syndromes; the management of specific complications of cholestasis, such as pruritus, fatigue and hyperco- lesterolemia and special controversial topics including management of recurrent cholangitis, prevention and management of biliary tract tumors in PSC and liver transplantation (LT) for AIH, PSC and PBC. Twenty panelists were chosen to elaborate a concise document of those topics after appropriate review of the literature, as well as to present the available data with the preliminary recommendations during the meeting. The resulting recommendations were discussed during the meeting, as well as afterwards, with all members of the Brazilian Society of Hepatology, which were invited to collaborate with suggestions through the society homepage. The present manuscript is the final version of the document followed by the recommendations which were graded according to the grading system adopted by the American College of Cardiology and the American Heart Association, as outlined below ${ }^{(1,2)}$.

\footnotetext{
Declared conflict of interest of all authors: none

${ }^{1}$ Hospital Português, Salvador, BA, Brasil; ${ }^{2}$ Faculdade de Medicina da Universidade de São Paulo, SP, Brasil; ${ }^{3}$ Faculdade de Medicina da Universidade Federal de Minas Gerais, MG, Brasil; ${ }^{4}$ University of Miami, USA; ${ }^{5}$ Faculdade de Medicina da Universidade Federal de São Paulo, SP Brasil; ${ }^{6}$ Hospital Israelita Albert Einstein, SP, Brasil; ${ }^{7}$ Centro de Ciências da Saúde da Universidade Federal do Espírito Santo, Vitória, ES, Brasil; ${ }^{8}$ Hospital Universitário da Faculdade de Medicina da Universidade Federal de Espírito Santo, ES, Brasil; Faculdade de Medicina da Universidade Federal de Pernambuco, PE, Brasil; ${ }^{9}$ Departamento de Gastroenterologia da Universidade Federal de Santa Catarina, SC, Brasil; ${ }^{10}$ Universidade Estadual de Campinas, SP, Brasil. ${ }^{*}$ Roberto José de Carvalho Filho ${ }^{5}$. Dalton Marques Chaves ${ }^{2}$, Irene Kazue Miura ${ }^{2}$, Liana Codes ${ }^{1}$, Luciana Costa Faria ${ }^{3}$. Andreia Silva Evangelista ${ }^{6}$, Alberto Queiroz Farias ${ }^{2}$, Luciana Lofêgo Gonçalves ${ }^{7}$, Michele Harriz², Edmundo Pessoa A Lopes Neto ${ }^{8}$, Gustavo Oliveira Luz², Patrícia Oliveira ${ }^{5}$, Elze Maria Gomes de Oliveira ${ }^{5}$, Janaina Luz Narciso Schiavon ${ }^{9}$, Tiago Seva-Pereira ${ }^{10}$, Edison Roberto Parise ${ }^{5}$

Correspondence: Paulo Lisboa Bittencourt. Rua Prof. Clementino Fraga, 220, 1901 - CEP: 40170050 - Salvador, Bahia, Brasil. Email: plbbr@uol.com.br
} 
- Class I: conditions for which there is evidence and/ or general agreement that a given diagnostic evaluation, procedure or treatment is beneficial, useful, and effective.

- Class II: conditions for which there is conflicting evidence and/or a divergence of opinion about the usefulness/efficacy of a diagnostic evaluation, procedure, or treatment.

- Class IIa: weight of evidence/opinion is in favor of usefulness/efficacy.

- Class IIb: usefulness/efficacy is less well established by evidence/opinion.

- Class III conditions for which there is evidence and/ or general agreement that a diagnostic evaluation, procedure/treatment is not useful/effective and in some cases may be harmful.

\section{PART I: AUTOIMMUNE HEPATITIS}

\section{Clinical manifestations}

Autoimmune hepatitis (AIH) is a chronic inflammatory liver disease characterized by hipergamaglobulimemia and reactivity autoantibodies, particularly for anti-smooth muscle (SMA), antinuclear (ANA), anti-liver kidney microsome type 1 (anti-LKM1), anti-soluble liver antigen (anti-SLA) and anti-liver cytosol type 1 (anti-LC1) antibodies. AIH primarily affects women and typically responds to immunosuppressive therapy with clinical, biochemical and histological remission. Frequently, the diagnosis of AIH is made when in the presence of inespefic clinical symptoms, like fatigue, nausea, upper abdominal pain, rash, arthralgia and oligomenorrhea. Jaundice can be present in half of subjects and nearly $1 / 3$ of patients can present with signs of advanced liver disease, such as ascites, splenomegaly, variceal bleeding and hepatic encephalopathy.

The disease can resemble an acute hepatitis in approximately $30 \%$ of cases with symptoms of jaundice, choluria, fecal acholia, and eventually, pruritus, especially in young girls. Fulminant hepatic failure (FHF) can also be a manifestation of severe AIH, and the disease should be considered in the differential diagnosis of FHF. Nearly $15 \%-20 \%$ of the patients can be asymptomatic at the time of diagnosis that may be elicited due to the biochemical abnormalities, detected during check-up evaluations, or in patients with other extrahepatic autoimmune disorders, like thyroiditis, arthritis or diabetes mellitus ${ }^{(3,4)}$.

The disease is usually classified in two types based on the autoantibody profile. Autoimmune hepatitis type 1 (AIH-1) is defined by the presence of SMA and/or ANA, whereas AIH type 2 (AIH-2) is characterized by reactivity for anti-LKM1 and/or anti-LC1. Diagnostic criteria and treatment options do not differ between both groups, but patients with AIH-2 may have distinct clinical features at onset such as younger age, higher frequency of acute liver failure, lower gammaglobulin levels and an increased incidence of $\operatorname{IgA}$ deficiency $\mathrm{y}^{(3,4)}$.

A comparative study of 115 Brazilian and 161 North-
Americans patients with AIH-1 showed that Brazilian patients had earlier disease onset, lower frequency of concurrent immune diseases, higher serum aspartate aminotransferase (AST) and gammaglobulin levels, greater occurrence of smooth muscle antibodies (SMA), and lower frequency of antinuclear antibodies (ANA), when compared to their North-American counterparts. Besides, HLA-DR 13 occurred more commonly in Brazilian patients, when compared to North-American subjects, who had more often HLA-DR3 and -DR4 ${ }^{(5)}$.

Data from a Brazilian cohort from the São Paulo University showed that patients with AIH $(n=268)$ had more frequently at disease onset clinical manifestation resembling acute hepatitis $(56 \%)^{(6)}$.Signs and symptoms of advanced chronic liver disease (CLD) were present in $25 \%$ of them and $10 \%$ were asymptomatic at diagnosis. Differences in clinical presentation between studies may be in part due to distinct genetic background but also to heterogeneity between parameters employed by the authors to evaluate types of clinical presentation. When the disease begins in the childhood, patients more frequently have a acute clinical course and a higher frequency of cirrhosis at diagnosis $(60 \%-80 \%)^{(7)}$.

Brazilian patients with AIH-1 with less than 18 years, when compared to older subjects had higher frequency of acute disease, SMA reactivity and HLA-DR13 as well as a lower frequency of concurrent extrahepatic autoimmune diseases. Patients older than 18 years also showed similar clinical features of their North American conterparts regarding the autoantibody profile and the frequency of concurrent extrahepatic autoimmune ${ }^{(5)}$.

A variant of $\mathrm{AIH}$, called autoimmune sclerosing cholangitis (ASC), characterized by the concomitance of colangiografic findings of PSC and AIH in the same patient, has been frequently described in children ${ }^{(8)}$. Even though this variant has been also reported in adults whether it is a different entity of part of AIH spectrum is not known. It would be better characterized elsewher in this manuscript as part of the spectrum of AIH and PSC overlap syndrome.

Autoimmune hepatitis can cause FHF in $2 \%$ to $16 \%$ of patients. Those frequencies may be underestimated as patients could also be erroneously classified as cryptogenic FHF due to the fact that most of them do not have the fullblown AIH phenotype. The frequency of FHF cause with unknown etiology varies from $16 \%-47 \%$, reaching $57 \%$ of the cases in $\operatorname{Japan}^{(9)}$.

Association of AIH with other extrahepatic autoimmune diseases has been reported in $30 \%-50 \%$ of the AIH patients. The most frequent reported disorders were thyroiditis, either Hashimoto thyroiditis or Graves disease, and rheumatoid arthritis, but type 1 diabetes mellitus, Sjogren Syndrome, polymyositis, IgA deficiency, idiopathic thrombocytopenic purpura, urticaria, vitiligo, Addison's disease, inflammatory bowel diseases, celiac disease have also been associated with AIH. Sero-negative or rheumatoid arthritis and thyroiditis were the most frequent associated autoimmune diseases in Brazilian patients with AIH type I and AIH type II, respectively ${ }^{(1,2,10)}$. 
Bittencourt PL, Cançado ELR, Couto CA, Levy C, Porta G, Silva AEB, Terrabuio DRB, Members of the Pannel of the 1st Consensus of the Brazilian Society of Hepatology on the Diagnosis and Management of Autoimmune Diseases of the Liver. Brazilian Society of Hepatology recommendations for the diagnosis and management of autoimmune diseases of the liver

\section{Recommendations}

- In the majority of cases, patients with AIH have unrecognized CLD with acute hepatitis-like symptoms, but signs and symptoms of advanced CLD may also be present. Less frequently, the disease can be present without symptoms or with FHF (Class IIa).

- AIH primarily affects women in a 4:1 ratio, mostly in 5 to 25 years of age, but it can occur in all age and races (Class IIa).

- Autoimmune extrahepatic disorders are frequently seen in patients with AIH. Autoimmune thyroiditis and rheumatoid arthritis are the most common encountered diseases. (Class I)

\section{Diagnosis}

The diagnosis of AIH is based on clinical, biochemical, serological and histological features and requires the exclusion of other causes of liver disease, such as viral hepatitis, hemochromatosis, Alpha-1 antitrypsin deficiency, druginduced liver diseases, Wilson's disease, non-alcoholic fatty liver disease and alcohol ${ }^{(11,12)}$.

Aminotransferase levels can be more than 10 times the upper limit of normal (ULN), the alkaline phosphatase (ALP) levels in general are less than three times the ULN, while the gamaglutamiltranspeptidase (GGT) levels are quite variable. Hypergammaglobulinemia, with a predominance of the $\mathrm{IgG}$ fraction, is the hallmark of the disease ${ }^{(12,13)}$.

Determination of circulating autoantibodies (AA) are key to the diagnosis and characterization of ALD. Most frequently, AA are non-organ specific and its expression can vary during the course of the disease, may also be present in other infectious liver diseases, rheumatological diseases, or even absent in $10 \%$ of cases $^{(11,14)}$. ANA, ASMA, anti-LKM1 are the classical AA used to diagnose and classify AIH. They are tested by indirect immunofluorescence (IFI) using tissue sections of kidney, liver and stomach of rodents as a substrate $^{(15)}$. Titers of AA in general do not correlate with severity of disease and its clinical course. Titers of SMA, but not ANA, are useful markers of disease activity and can be used to monitor response to treatment ${ }^{(3,16)}$.

According to international criteria, AA titers are considered positive, when present at a dilution of at least 1:40 in adults, while titers of at least 1:20 for SMA and ANA or titers of at least 1:10 for anti-LKM1 are considered positive in children ${ }^{(17)}$. Homogeneous and speckled are the most frequent patterns of ANA in AIH patients ${ }^{(11,13,15,18)}$. Centromeric, nucleolar and nuclear dots cannot be considered as markers of AIH.

SMA reactivity, with or without ANA, characterizes AIH-1. Both can turn to be negative after treatment ${ }^{(3,16)}$. The immunofluorescent staining of SMA is detected in the arterial walls of rodent kidney, liver and stomach. In the kidney, SMA can have three patterns: SMA-V (vessels), SMA-VG (vessels and glomeruli) and SMA-VGT (vessels, glomeruli and tubules). SMA-VG and AMA-VGT patterns are more specific of AIH, but SMA-V pattern is frequently found in viral hepatitis ${ }^{(19,20)}$. The specificity for antigens present in the microfilaments (F-actin), characterizing anti-actin antibody (AAA) is specific, but not pathognomonic, of AIH and is well correlated with the presence of SMA-VGT pattern in IIF ${ }^{(21)}$.

Lack of IFI standardization limits its use on routine ba$\operatorname{sis}^{(13,15)}$ Usually, patients with reactivity for SMA are younger than patients with reactivity for ANA. In this regard, patients with AIH-1 and SMA also exhibit reactivity for AAA and are shown to carry HLA-DR 13 and -DR3 genotypes in South America and HLA-DR3 and -DR4 in Europe and North-America.

Autoimmune hepatitis type 2 is characterized primarily by reactivity to anti-LKM1 ${ }^{(21,22)}$. It reacts with CYP2D6 antigens, but can be positive in up to $5 \%$ of hepatitis C patients ${ }^{(23)}$.As previously pointed out, patients with AIH-2 are younger, have lower levels of gamaglobulin, carry more frequently HLA-DR 7 and/or -DQ2 genotypes and are more prone to have FHF at presentation, when compared to their counterparts with AIH-1 ${ }^{(24,25)}$.

Other AA can be tested to better characterize AIH patients without those aforementioned conventional antibodies. Anti-soluble liver pancreas (Anti-SLA/LP), that the molecular target is as $\operatorname{Sep}(\mathrm{O}$-phosphoserine) tRNA:Sec(selenocysteine)tRNA synthase (SEPSECS) is highly specific for AIH but has low sensitivity ${ }^{(26,27)}$.It is mostly detected in asociation with ANA and SMA. Its presence identifies patients with a more severe disease and a worse outcome. It is also associated with HLA-DR $3^{(28,29)}$.Anti-LC1 is the second marker of AIH-2, detected in $24 \%$ to $32 \%$ of patients with anti-LKM1 and rarely as the only marker of AIH-2. It occurs mainly in young patients with an aggressive disease course with rapid progression to cirrhosis ${ }^{(30,31)}$. The perinuclear anti-neutrophil cytoplasm (pANCA) antibody and anti-asialoglycoprotein receptor antibody (ASGPR) are less frequently used in clinical practice since they are not specific for the diagnosis of $\mathrm{AIH}^{(29,32)}$.

To better standardize the diagnosis of AIH, the International AIH Study Group (IAIHSG) have stablished a scoring system in 1993 to define AIH as probable or definite $^{(12)}$. (Table 1). The scoring system was first updated in 1999, to better exclude those patients with diagnosis of PSC and PBC. In order to improve its clinical application, a simplified scoring system was then proposed in 2008 with only four independent variables, including autoantibodies, IgG levels, histology and absence of viral markers (Table $2)^{(33)}$. The revised AIHSG, as well as the simplified criteria, were shown to have higher sensitivity and specificity for the diagnosis of AIH and are particularly useful in different clinical scenarios. In patients with atypical features of AIH, cryptogenic cirrhosis or AIH without conventional autoantibodies, the revised IAIHSG criteria are very helpful for stablishing the diagnosis of AIH. On the other hand, the simplified criteria is valuable in excluding AIH in subjects with other liver diseases and concurrent extrahepatic autoimmune disorders ${ }^{(34)}$. However, they do not take into account treatment responses or relapse after treatment withdrawal or the presence of clinical or laboratory features of other liver diseases. 
TABLE 1. International Autoimmune Hepatitis Study Group scoring system for the diagnosis of autoimmune hepatitis

\begin{tabular}{|c|c|}
\hline Parameters & Score \\
\hline Female sex & +2 \\
\hline \multicolumn{2}{|l|}{ ALP / ALT ratio (xULN) } \\
\hline$<1,5$ & +2 \\
\hline $1.5-3.0$ & 0 \\
\hline$>3.0$ & -2 \\
\hline \multicolumn{2}{|l|}{ Globulins, Gammaglobulins or IgG (xULN) } \\
\hline$>2.0$ & +3 \\
\hline $1.5-2.0$ & +2 \\
\hline $1.0-15$ & +1 \\
\hline$<1.0$ & 0 \\
\hline \multicolumn{2}{|l|}{ Autoantibodies (IIF titers in rodent tissues) } \\
\hline \multicolumn{2}{|l|}{ Adults: ANA,SMA and anti-LKM1 } \\
\hline$>1 / 80$ & +3 \\
\hline $1 / 80$ & +2 \\
\hline $1 / 40$ & +1 \\
\hline$<1 / 40$ & 0 \\
\hline Children: ANA, SMA, anti-LKM1 > 1/20 & +3 \\
\hline Children: ANA, anti-LKM1:1/10-1/20 & +2 \\
\hline Children: SMA $1 / 20$ & +2 \\
\hline Children: SMA 1/10 & +1 \\
\hline \multicolumn{2}{|l|}{ Viral markers } \\
\hline Anti-HAV IgM, AgHBs or antiHBc IgM positive & -3 \\
\hline Anti-HCV e RNA do HCV positivos & -3 \\
\hline $\begin{array}{l}\text { Anti-HAV IgM, AgHBs, anti-HBc IgM or anti-HCV } \\
\text { negative }\end{array}$ & +3 \\
\hline Recent use of hepatotoxic drugs positive/negative & $-4 /+1$ \\
\hline Alcohol intake: $<25 \mathrm{~g} /$ day & +2 \\
\hline$>60 \mathrm{~g} /$ day & -2 \\
\hline $\begin{array}{l}\text { Other autoimmune disease in the patient or first degree } \\
\text { family member }\end{array}$ & +2 \\
\hline Histology: Interface hepatitis & +3 \\
\hline Rosettes & +1 \\
\hline $\begin{array}{l}\text { Severe lymphoplasmocytic infiltrate Infiltrado } \\
\text { inflamatório }\end{array}$ & +1 \\
\hline None of histological findings above & -5 \\
\hline Biliary changes suggestive of $\mathrm{PBC}$ or PSC & -3 \\
\hline Other histological features suggestive of other etiology & -3 \\
\hline \multicolumn{2}{|l|}{$\begin{array}{l}\text { Other autoantibodies in subjects without ANA, SMA and } \\
\text { anti-LKM1 }\end{array}$} \\
\hline $\begin{array}{l}\text { Anti-SLA/anti-LP, anti-LC1, anti-liver specific protein, } \\
\text { anti-asialoglycoprotein receptor: positive/negative }\end{array}$ & $+2 / 0$ \\
\hline $\begin{array}{l}\text { HLA-DR13 and -DR3 for AIH-1 or HLA-DR7 and } \\
\text {-DR3 for AIH-2 }\end{array}$ & +1 \\
\hline \multicolumn{2}{|l|}{ Treatment response } \\
\hline Complete & +2 \\
\hline $\begin{array}{l}\text { Relapse during or after withdrawal of therapy after a } \\
\text { complete response }\end{array}$ & +3 \\
\hline Definite diagnosis: before treatment & $>15$ \\
\hline Definite diagnosis: after treatment & $>17$ \\
\hline Probable diagnosis: before treatment & $10-15$ \\
\hline Probable diagnosis: after treatment & $12-17$ \\
\hline
\end{tabular}

TABLE 2. Simplified criteria for the diagnosis of AIH

\begin{tabular}{|c|c|c|}
\hline Parameters & & Escore \\
\hline ANA or SMA & $1 / 40$ & +1 \\
\hline ANA or SMA & $1 / 80$ & +2 \\
\hline anti-LKM1 & $\geq 1 / 40$ & +2 \\
\hline anti-SLA & Positive & +2 \\
\hline \multirow{2}{*}{$\operatorname{IgG}$} & $>1 \times U L N$ & +1 \\
\hline & $>1,1 \times \mathrm{ULN}$ & +2 \\
\hline \multirow{2}{*}{ Histology } & Compatible & +1 \\
\hline & Typical & +2 \\
\hline Virological serology & Negative & +2 \\
\hline Definite diagnosis & & $\geq 7$ \\
\hline Probable diagnosis & & 6 \\
\hline
\end{tabular}

Liver biopsy should be performed, whenever possible, to confirm the diagnosis of $\mathrm{AIH}$, to exclude other liver diseases as well as to guide therapy. It can be valuable also to stablish prognosis, as $30 \%-50 \%$ of the patients with AIH have cirrhosis at disease onset ${ }^{(11,12)}$. Typical, but not patognomonic, histologic features of AIH include the presence of interface hepatitis, moderate to severe lymphoplasmocytic portal infiltrate with rosetting of hepatocytes, varying degrees of lobular inflammation, bridging necrosis and sometimes multiacinar collapse. Granulomas are exceptional, as well as, severe bile duct lesions. Liver biopsy is considered a prerequisite for the diagnosis of AIH and is also helpful for assessment of portal inflammation and fibrosis staging. Noninvasive tests have gained currently widespread use in the quantification of fibrosis in patients with hepatitis $\mathrm{C}$ and nonalcoholic steatohepatitis. However, they have not been validated for assessment of fibrosis in AIH and it could be hypothesized that their use in this setting would be hampered by the inflammatory activity seen in this disease.

Autoimmune hepatitis frequently has an acute presentation, sometimes FHF, resembling acute hepatitis or druginduced liver disease. Patients with severe acute hepatitis or FHF may have autoantibodies in low-titer or absence of conventional autoantibodies. They may also have normal gammaglobulin or IgG levels and atypical histological findings, including zone III centrolobular necrosis, perivenullar or central inflammation with lymphocytes and plasma cells, multiacinar collapse and lymphoid aggregates ${ }^{(9,35,36)}$. Diagnostic scoring systems can aid in the diagnosis of these peculiar types of $\mathrm{AIH}$, but it should be kept in mind that they have been standardized in cohorts of subjects with classical AIH. Sometimes, when liver biopsy is unfeasible, a short trial of corticosteroids is warranted in those difficult cases in order to stablish or refute the diagnosis of AIH based on the treatment response. 
Bittencourt PL, Cançado ELR, Couto CA, Levy C, Porta G, Silva AEB, Terrabuio DRB, Members of the Pannel of the 1st Consensus of the Brazilian Society of Hepatology on the Diagnosis and Management of Autoimmune Diseases of the Liver. Brazilian Society of Hepatology recommendations for the diagnosis and management of autoimmune diseases of the live

\section{Recomendations}

- The diagnosis of AIH should be performed in patients with elevated aminotransferases and gammaglobulin levels, reactivity for SMA, ANA, anti-LKM1, antiLC1 and anti-SLA and typical histological findings, after the exclusion of other liver disease, particularly viral hepatites and Wilson's disease (Class I)

- The revised IAIHSG scoring system and the simplified AIH criteria can be used for the diagnosis of AIH, but the former performs better in the diagnostic evaluation of atypical cases (Class IIa).

- Liver biopsy, whenever possible, should be perfomed in patients with AIH for histological diagnosis and prognostic assessement. However, it may not be entirely necessary in patients with classical full-blown disease (Class IIa).

- SMA, ANA, anti-LKM1 and anti-LC1 should be screened by indirect imunofluorescence using rodent tissues, while anti-SLA reactivity should be assessed by ELISA or immunoblotting (Class I).

\section{Management and treatment of AlH}

Treatment of AIH should begin preferably with dual drug therapy with azathioprine and corticosteroids, either prednisone or prednisolone in daily doses, respectively, of $50 \mathrm{mg}$ and $30 \mathrm{mg}$. The American Association for the Study of Liver Diseases (AASLD) guidelines recommend weekly tapering of prednisone, but most centers in Brazil, probably due to the severity of AIH in our country, prefer to gradually reduce the dose of prednisone at monthly intervals ${ }^{(11)}$. The treatment protocol at the University of São Paulo advocate reduction of prednisone after one month from $30 \mathrm{mg}$ to $20 \mathrm{mg}$ per day, if aminotransferase levels decrease, maintaining the dosage of azathioprine in 50 $\mathrm{mg}$ per day. After the second month, if aminotransferases keep falling, prednisone is decreased to achieve levels of $10 \mathrm{mg}$ per day by 6 months. On the contrary, azathioprine is gradually increased up to $2 \mathrm{mg} / \mathrm{kg} /$ day $(75-150 \mathrm{mg} /$ day), particularly if there is no change or an increase in either AST or ALT or requirement for increased dosages of corticosteroids. Futher adjustments may be needed according to tolerance and staging of AIH and it is entirely acceptable to keep doses of prednisone and azathioprine around 15 $\mathrm{mg} /$ day and $150 \mathrm{mg} /$ day, respectively, to achieve and keep aminotransferases in the normal range ${ }^{(37)}$. Monotherapy with prednisone is rarely employed due to the adverse side effects of high doses of corticosteroids, in the absence of allergy to azathioprine or drug intolerance. Whenever required, corticosteroid monotherapy is initially introduced with prednisone $60 \mathrm{mg} /$ day with dose reductions to $40 \mathrm{mg} /$ day and then $30 \mathrm{mg} /$ day every two weeks depending on the levels of AST and/or ALT. After the third month of therapy, the drug is reduced to maintenance doses of $20 \mathrm{mg} /$ day. In the presence of normal aminotransferases, further reductions to $10-15 \mathrm{mg} /$ day of prednisone monotherapy may be attempted, but remission is rarely maintained with those doses of corticosteroids.
In order to prevent or reduce corticosteroid side effects, budesonide has been initially evaluated in a pilot study from the Mayo Clinic without satisfactory results ${ }^{(38)}$. However, one subsequent multicentre randomized controlled trials (RCT), comparing azathioprine and budesonide vs. azathioprine and prednisone, disclosed higher rates of remission and less side effects in the group of budesonide treated patients. However, overall rates of remission in this RCT, $60 \%$ in the budesonide-treated patients vs. $39 \%$ in the prednisone-treated subjects, were much lower, when compared to previously reported treatment outcomes. It should also be stressed that prednisone was rapidly tapered in this RCT, in accordance with AASLD guidelines, whereas stardand doses of 6-9 $\mathrm{mg} /$ day of budesonide were maintained throughout the study ${ }^{(39)}$. Budesonide is contraindicated in patients with cirrhosis and also in subjects with portal hypertension due to an increased risk of portal vein thrombosis. It is also not advisable to employ the drug in AIH subjects with concurrent extrahepatic autoimmune disorders, that may benefit from prednisone treatment. Therefore, up to now it remains controversial whether budesonide should be preferred over prednisone in the first-line treatment of AIH due to uncertainty regarding and in adittion higher cost.

Measurement of azathioprine metabolytes may be useful in certain clinical settings to adjust its dosage and to look for patient's drug adherence. Two metabolytes can be measured: 6-thioguanine and 6-metilmercaptopurine. Therapeutic effects of azathyoprine are ascribed to 6-thioguanine as well as dose-related myelotoxicity, whereas hepatotoxicity of the drug is related to 6-metilmarcaptopurine. In this regard, levels of 6-thioguanine and 6-metilmercaptopurine should be maintained, respectively, in the range of $235-450 \mathrm{pmol} / 8$ $\mathrm{x} 10^{(8)}$ red blood cells and less than $5.700 \mathrm{pmol} / 8 \times 10^{(8)} \mathrm{red}$ blood cells ${ }^{(38)}$, to avoid suboptimal treatment responses as well as myelotoxicity and hepatotoxicity. There is no role for the measurement of those metabolytes in stable patients easily controlled with standard doses of azathioprine (75$100 \mathrm{mg}$ /day). However, their monitoring could be useful in those patients with poor treatment responses requiring higher doses of the drug. When both drug metabolytes are above the aforementioned levels, an increase in azathioprine dosage would be inapropriate, leading only to increased drug toxicity without benefical therapeutical effects. However, in the presence of lower 6-thioguanine and higher than desired levels of 6-mercaptopurine, adittion of allopurinol in doses of $100 \mathrm{mg} /$ day coupled with a $25 \%$ to $50 \%$ reduction in total daily dosage of azathioprine, was shown to swich metabolic drug pathways toward 6-thioguanine and increased drug efficacy and safety profile. It should be emphasized that this approach was better validated in patients with inflammatory bowel disease (IBD) but not in subjects with AIH. Close monitoring of drug metabolytes is mandatory during this treatment strategy $(40)$

Treatment of AIH is introduced to induce clinical remission, which is easily achieved in the first two months of therapy, as well as biochemical remission with normalisation of aminotransferases, gammaglobulins and $\mathrm{IgG}$ and 
restoration of albumin and bilirrubin leves and INR to normal values. Therapy is guided toward complete treatment response, defined by normalization of either AST and ALT as well as $\mathrm{IgG}$, which requires at least 6 months of immunossupression $^{(10)}$. Treatment is usually required for at least 24 months. Liver biopsy to assess histological remission is required before evaluation of treatment in patients with clinical or biochemical remission. Timing for liver biopsy is not standardized. Most centers recommend histological evaluation 18 months after biochemical remission. The presence of circulating SMA, and particularly AAA, but not other AA, have been associated with a higher risk for histological activity of AIH, which may guide the appropriate timing of liver biopsy in this setting ${ }^{(16)}$.

In the presence of histological remisson, with none of minimal portal inflammatory activity disclosed at liver biopsy, treatment withdrawal should be carefully evaluated and discussed with the patient, because relapse rates can be observed in up to $70 \%-80 \%$ of the subjects, requiring reintroduction of higher doses of immunossupression or leading to disease dacompensation in those subjects with cirrhosis and poor liver function. Relapse usually occurs insidiously in the first six months of treatment withdrawal and should be regularly monitored with periodic measurement of AST and $\mathrm{ALT}^{(4,41)}$.It should also be taken into account, the AA profile, since anti-SLA reactivity is associated with higher relapse rates $^{(26)}$. It is uncertain whether the treatment outcomes after relapse would be worse or better when compared to previous response to therapy ${ }^{(4)}$.

There are three management options after stablishment of clinical, biochemical and histological remission of AIH: 1) to withdraw treatment with the aim of achieving spontaneous long-term remission; 2) to continue the same treatment schedule to maintain remisson, 3) to change the immunossupressive regimen either maintaining azathioprine after the weaning of the corticosteroids or substituting both drugs to less toxic alternatives. In this regard, one pilot study has suggested that cloroquine would be a reasonable option ${ }^{(44)}$.

The IAIHSG has defined relapse in the presence of a two-fold increase in aminotransferases after withdrawal of treatment $^{(12)}$. This usually occurs insidiously in the first three months of follow-up. Slight increases of aminotransferases may be transient with spontaneous return to the normal limits thereafter. Thus, it is not stablished when and how treatment should be reinstituted after relapse. High doses of immunossupression may not be entirely required depending on the absence of symptoms and the degree of biochemical abnormalities.

There is no formal requirement in adjustment of $\mathrm{AIH}$ treatment during pregnancy and lactation, despite some reports on teratogenic effects, lymphopenia and tymic atrophy of newborns of women taking azathioprine ${ }^{(4)}$. Due to the fact that azathioprine is considered a class D drug for pregnancy by the FDA, some experts prefer to maintain only corticosteroids during pregnancy. In agreement, the policy of the University of São Paulo is to avoid azathioprine and to maintain prednisone $15-20 \mathrm{mg} /$ day until delivery ${ }^{(46)}$.
Due to the high levels of strogen encountered throughout pregnancy and the consequent switch of Th1 to Th2 immune responses, reduction of disease activity is generally observed during pregnancy with frequent $\mathrm{AIH}$ recrudescence during puerperium.

Either vaginal or cesarian delivery can be accomplished depending on the clinical conditions of the mother and the child. Vaginal delivery would be more appropriate in most subjects, particularly those without cirrhosis and portal hypertension. In their counterparts with large esophageal varices, not previously erradicated by banding, cesarian section would be more advised due to the risk of variceal hemorrhage during labor ${ }^{(47)}$.

According to medical litterature, treatment response with azathioprine and prednisone with clinical, biochemical and histological remission is observed in $70 \%-80 \%$ after 3 years of therapy. Recent data, employing revised criteria encompassing complete normalization of liver enzymes as the main endpoint, revealed much lower rates of treatment response, as low as $35 \%$ in 5 years in the experience of the University of São Paulo(6).

Autoimmune hepatitis refractory or with no response to treatment was reported in 7\%-9\% of the cases, whereas incomplete response was shown in around $10 \%-13 \%$ of treated subjects. In these patients, other treatment regimens have been evaluated, including calcineurin inhibitors, either cyclosporin or tacrolimus, mycophenolate mofetil, ursodeoxicholic acid (UDCA), anti-tumor necrosis factor (TNF) alpha agents as well as rituximabe. Some of those agents were also employed in the $10 \%-15 \%$ of subjects, who turn to be intolerant to either azathioprine or corticosteroids, due to the development of side effects ${ }^{(11,13,48-52)}$.

There is no evidence-based rational for the use of the aforementioned drugs, currently employed after LT, to treat AIH. Most studies evaluating their use were uncontrolled and non-randomized. There are several reports, comprising more than 100 patients, evaluating cyclosporin use in patients either with refractory AIH or without response or intolerance to azathioprine and/or prednisone. Improvement of liver enzymers was observed in $93 \%$ of the cases. Only $7 \%$ of those treated subjects were refractory or intolerant to cyclosporin $^{(11,13,48-52)}$. One study reported significant improvement in AST and/or ALT with good drug tolerance in 19 (nine treatment naïve) adult patients with AIH followed by 26 weeks. Cyclosporin was used aiming to achieve trough levels between 100-300 ng/ml ${ }^{(53)}$. Tacrolimus, on the other hand, have been employed for treatment of AIH since 1995 with improvement in liver enzymes reported in most of the treated patients $^{(13,48-52)}$. Both drugs share similar adverse events, but while diabetes, neurotoxicity, nefrotoxicity, diarrhea, pruritus and alopecia are more frequent with tacrolimus, arterial hypertension, dyslipidemia, hyrsutism and gengival hypertrophy are more commonly seen in cyclosporin-treated patients ${ }^{(48)}$. In adittion, cyclosporin is generally preferred over tacrolimus due to availability of more data favoring its use in AIH.

Mycophenolate mofetil has been employed in treatment naïve and azathyoprine intolerant patients as well as subjects 
with AIH non-responders to conventional treatment. In most of the studies, doses of mycophenolate mofetil ranged from 0,5 to $3 \mathrm{~g} /$ day (average $2 \mathrm{~g} /$ day). Treatment responses appeared to be better in patients with AIH intolerant to azathyoprine, when compared to their counterparts with refractory disease. Different from azathiyoprine, metabolism of mycophenolate is not related to 6-thioguanine metiltransferase. In one study evaluating mycophenolate mofetil in treatment-naïve patients with AIH, $88 \%$ of the subjects had biochemical remission in three months. Partial response, corticosteroid withdrawal were observed, respectively, in $12 \%$ and $58 \%$ of the patients ${ }^{(54)}$.Drug-related side effects, particularly nauseas, diarrhea and abdominal pain, were noted in $3 \%$ to $33 \%$ of the patients ${ }^{(4,48,50-52,54)}$. Despite its safety profile and efficacy, it can not be recommend as first-line therapy due to the paucity of data regarding its use and its higher cost in comparison to azathioprine.

Initial reports with the use of anti-CD20 antibody, rituximabe, in the treatment of AIH and concurrent extrahepatic autoimmune diseases such as idiopathic thrombocytopenic purpura, cryoglobulinemic glomerulonephritis and autoimmune hemolytic anemia were encouraging. One study included six patients with AIH either intolerant $(n=3)$ or refractory $(n=3)$ to conventional treatment. The treatment schedule proposed was $1 \mathrm{~g}$ of rituximabe at once and 14 days thereafter with maintenance of azathioprine and gradual weaning of corticosteroids for 72 months. All patients had marked biochemical and/or histological improvement. The drug was well tolerated without significant side effects ${ }^{(55)}$.

Anti-TNF alpha agents, such as infliximabe, etanercept and adalimumabe are commonly used for treatment of rheumatoid arthritis, psoriasis and IBD. Weiler-Normann et $a l^{(56)}$ have recently reported the use of infliximabe in 11 patients with refractory AIH. They have employed the drug, given as an intravenous infusion of $5 \mathrm{mg} / \mathrm{kg} / \mathrm{dose}$, at days one, 14 and 42 of therapy and repeated thereafter every four to six weeks, depending on the treatment response. Improvement in liver enzymes was seen in all patients. Biochemical and histological remission were achieved, respectively, in 8 patients and in all of the five subjects submitted to follow-up liver biopsy. These results were promising in this difficult-to-treat patients, but it caution is advised with the use of these agents, due to their enhanced risk to induce viral and bacterial infections, particularly in subjects with cirrhosis $^{(56)}$.

Ursodeoxicholic acid (UDCA) is a choleretic and immunomodulatory drug that was also evaluated in the treatment of AIH. One Japanese study have reported clinical, biochemical and histological remission with the use of $600 \mathrm{mg} /$ day of UDCA for treatment of patients with a less-aggressive form of $\mathrm{AIH}^{(57)}$.Another American study failed to confirm the aforementioned results ${ }^{(58)}$. On the other hand, UDCA were reported to be beneficial when added to conventional treatment with azathyoprine and prednisone in those patients with abnormal ALT and GGT, leading to normalization of these liver enzymes in $67 \%$ of the AIH treated subjects ${ }^{(6)}$.

\section{Features of AlH in children}

Children with platelet and leukocyte counts, respectively, above $50.000 / \mathrm{mm}^{(3)}$ and $3.000 / \mathrm{mm}^{(3)}$ should be treated with dual therapy with prednisone $1,5-2 \mathrm{mg} / \mathrm{kg} /$ day to a maximum dose of $60 \mathrm{mg} /$ day and azathyoprine $1-2 \mathrm{mg} / \mathrm{kg} /$ day. Treatment protocol of the University of São Paulo advocate tapering every 4-6 weeks the dose of prednisone, with a 50\% dose reduction in the first subsequent consultation with gradual decreases thereafter to achieve maintenance dosages of 2,5 to $5 \mathrm{mg} /$ day. Azathyoprine dosage can be adjusted according to treatment response and the development of side effects, such as leukopenia and trombocytopenia.

In children with platelet and leukocyte counts, respectively, below $50.000 / \mathrm{mm}^{(3)}$ and $3.000 / \mathrm{mm}^{(3)}$, monotherapy with prednisone is preferable in doses of $1,5-2 \mathrm{mg} / \mathrm{kg} /$ day with gradual tapering, as described above, until normalization of aminotransferases, gammaglobulins or $\mathrm{IgG}^{(59)}$.

The use of budesonide for AIH in childhood is unsettled. The is only one RCT with a small sample size comparing budesonide vs. prednisone for treatement of AIH. In this RCT, rates of were similar in subjects treated with either budesonide or prednisone. However, side effects were less often observed in the budesonide treated group of children. Due to the limited experience with the use of budesonide, it can not be recommneded for treatment of AIH in children ${ }^{(60)}$. There is also scarcity of data regarding the employment of other immunossupressors in pediatric AIH. Most published studies were uncontrolled and not randomized. In this regard, cyclosporin was associated with clinical and biochemical remission when used in children with AIH without cirrhosis or decompensated liver disease ${ }^{(61)}$.Unfavorable side effects were observed, including nefrotoxicity, hysurtism, tumors, dislipidemia and arterial hypertension. The efficacy and safety profile of tacrolimus was not adequately evaluated ${ }^{(62)}$. Mycophenolate mofetil, on the other hand, may be used in subjects with intolerance to azathioprine in association with prednisone ${ }^{(63)}$.

Anti-CD20 antibodies (rituximabe) may be employed to rescue children with AIH refractory to conventional treatment. Remission can be achieved in this difficult to treat patients under rituximabe, but experience with this drug in this setting is very limited ${ }^{(64)}$.

Criteria for evaluation of treatment response in $\mathrm{AIH}$ in children should include disappearence of symptoms, normalization of liver enzymes, gammaglobulins and IgG levels and no or minimal portal inflammation on liver biopsy ${ }^{(65)}$. Children should be treated for at least 24 months. Liver biopsy to evaluate histological remission, is mandatory after treatment withdrawal in patients with AIH-1. Some experts suggest maintenance of immunossupression in patients with AIH-2 even in the presence of histological remission due to their higher rate of relapse after interruption of treatment.

\section{Recommendations}

Initial treatment of AIH in adults should be instituted with dual therapy with azathioprine and prednisone in doses, respectively, of $30 \mathrm{mg} /$ day and $50 \mathrm{mg} /$ day, in the absence of known contraindications for the use of 
those drugs (Class I). In childhood AIH, dual therapy with prednisone $1,5-2 \mathrm{mg} / \mathrm{kg} /$ day (up to $60 \mathrm{mg} /$ daily) and azathioprine $1-2 \mathrm{mg} / \mathrm{kg} /$ day is also recommended (Class I).

- Despite the lack of data to guide drug adjustments during immunossupressive therapy of $\mathrm{AIH}$, it is suggested to taper the dose of prednisone at monthly intervals and to progressively increase the dose of azathioprine to achieve biochemical remission with as minimal side effects as possible with a median mantainance dose of prednisone and azathioprine, respectively, of 7,5-15 $\mathrm{mg} /$ day and $75-150 \mathrm{mg} /$ day, not exceeding doses of azathioprine greater than $2 \mathrm{mg} / \mathrm{kg} /$ day. Maintenance doses of those immunossupreisve drugs in children are usually $2,5-5 \mathrm{mg} / \mathrm{day}$ for prednisone and up to $2 \mathrm{mg} / \mathrm{kg} /$ day for azathioprine (Class IIb)

- It is suggested to begin monotherapy with prednisone in AIH adult patients with contraindications to azathioprine therapy. Treatment should begin with prednisone $60 \mathrm{mg} /$ day with subsequent tapering to $40 \mathrm{mg} /$ day and then $30 \mathrm{mg} /$ day every two weeks. The corticosteroid dose should be decreased more gradually afterwards to maintenance levels not higher than $20 \mathrm{mg} /$ day. In children, doses of corticosteroids should be tapered to achieve biochemical remission with minimal side effects.

- Despite one RCT demonstrating advantages of budesonide over prednisone in the treatment of $\mathrm{AIH}$, the use of budesonide as first-line therapy of AIH in adults, as well as in children cannot up to now be recommended (Class IIb).

- Clinical, biochemical and histological remission of AIH should be regarded as the primary end-point of treatment (Class I). In order to achieve this primary end-point, treatment should be maintained for at least 24 months. Liver biopsy should be performed at least 18 months after biochemical remission in order to assess histological remission (Class I).

- In patients with clinical, biochemical and histological remission, treatment withdrawal may be tried, after discussion of the benefits and risks with the patient. Close monitoring of AIH patients weaned off imunossupression is mandatory. Alternatively monotherapy with azathioprine in doses up to $2 \mathrm{mg} / \mathrm{kg} / \mathrm{day}$ may be instituted as maintenance treatment indefinitevely (Class IIa)

- In AIH patients with intolerance to azathioprine or suboptimal responses to dual conventional therapy, measurement of azathioprine metabolytes can be useful to perform drug adjustments as well as to add alupurinol to swich drug metabolism to 6-thyoguanine, which is more safe and effective, when compared to azathioprine. Alternatively mycophenolate mofetil can be used in substitution for azathioprine (Class IIb).

- Either cyclosporin or tacrolimus may be used in AIH patients without response to conventinal treatment, but cyclosporin is usually prefered due to a larger experience with the use of this drug in refractory AIH (Class IIa).

\section{PART II: PRIMARY SCLEROSING CHOLANGITIS}

\section{Diagnosis}

Primary sclerosing cholangitis is a chronic cholestatic liver disease of unknown cause characterized by diffuse inflammation, fibrosis and stenosis of the intrahepatic and/ or extrahepatic biliary tree ${ }^{(66)}$. The disease is considered to be immune-mediated but its etiopathogenesis is largely unrecognized. Genetic predisposition to PSC is complex, but is primarily linked to the major histocompatibility complex on chromosome $6^{(67)}$. It occurs more commonly in males, with a male to female ratio of $2: 1$, with a mean age at diagnosis around 40 years $^{(68)}$.

The clinical presentation of PSC is variable. Nowadays, most patients are entirely asymptomatic at diagnosis, which is usually carried out due to investigation of abnormal liver enzymes, particularly ALP and GGT, typically in a male adult patient with IBD. Other clinical features include pruritus, right upper quadrant pain, fatigue, weight loss as well as fever and chills associated with bacterial cholangitis ${ }^{(69)}$.

The clinical course of PSC may be complicated by the development of dominant stenosis in the biliary tree, cholangiocarcinoma (CC), gallbladder cancer, and colorectal cancer as well as decompensation of CLD including those manifestations of portal hypertension and end-stage liver failure. Symptoms attributable to chronic cholestasis such as fatigue, spontaneous fractures due to osteoporosis and pruritus may predominate in some subjects. Most of the patients with PSC either die due to liver failure, $\mathrm{CC}$ and colorectal neoplasia.

Primary sclerosing cholangitis usually affects the entire biliary tree. Aproximately $20 \%$ of the patients may have involvement restricted to the intrahepatic bile ducts and $5 \%$ of them only involvement of interlobular and septal bile ducts, which characterizes small-duct PSC, a condition with better prognosis defined by liver biopsy in a patient with IBD and a normal cholangiogram ${ }^{(70)}$.

There is a strong association of PSC with IBD. Either ulcerative cholitis (UC) or Crohn's disease (CD) are observed in $70 \%$ to $80 \%$ of the patients with PSC, but most of the cases of IBD in subjects with PSC are due to UC $(80 \%)$. Indeterminate colitis and $\mathrm{CD}$ are responsible each for $10 \%$ of the remaining cases $^{(71)}$.

Other autoimmune or immune-mediated disorders have been also associated with PSC, including AIH, celiac disease, rheumatoid arthritis, Sjögren syndrome, glomerulonephritis, systemic lupus erithematosus, autoimmune hemolytic anemia and idiopathic thrombocytopenic purpura. It is not clear however, whether those conditons are true associations or heve been merely encountered in PSC patients by chance ${ }^{(72)}$.

Patients with PSC and concurrent IBD are more often males, tend to be asymptomatic at diagnosis and to exhibit intra and extrahepatic involvement of the bliary tree. On the other hand, IBD, in subjects with PSC, is usually oligosymptomatic, tend to have rectal sparing and lower requirement for colectomy in the log-term. On the contrary, PSC subjects with $\mathrm{CD}$ are more commonly females and more often have small-duct PSC ${ }^{(70)}$. 
Cholangitis due to IgG4 is the hepatobiliary manifestation of multisystemic IgG4 disease, a fibroinflammatory disorder with variable clinical features, maily affecting the biliary ducts and the pancreas, leading to autoimmune pancreatitis and pancreatic pseudotumor. The disease occurs predominantly in older men, is frequently associated with lymphadenopathy, and responds well to steroid therapy. They often present more abruptly with painless obstructive jaundice, whereas obstructive jaundice is rarely present in PSC patients. It can be mistaken for pancreatic or bile duct cancer, as well as primary or secondary sclerosing cholangitis. Clinical manifestations are apparent in the pancreas, bile duct, gallbladder, salivary gland, retroperitoneum, kidney, lung and prostate, in which tissue fibrosis with obliterative phlebitis is pathologically induced.

Serum IgG4 levels and immunostaining for anti-IgG4 antibody in tissue specimens are useful in making the diagnosis. IgG4-related sclerosing cholangitis is not associated with IBD. In many cases, stenosis is located in the lower part of the common bile duct, but thickening of the common bile duct wall is sometimes detected even in the segment in which abnormalities are not clearly observed upon cholangiography ${ }^{(73,74)}$.

The diagnosis of PSC is usually stablished in subjects with clinical and laboratory features of cholestasis with typical cholangiographic findings of the disease either at MRCP or $\mathrm{ERC}$, including the presence of strictures alternating with dilatations or sacculations of intrahepatic and/or extrahepatic bile ducts ${ }^{(75)}$.

Abnormal levels of ALP and GGT are usually found in PSC subjects. An increase of AST and/or ALT levels, less than 2 to 3 times the upper limit of normal, is frequently seen in most patients, but normal AST/ALT levels can also be found. In this regard, ALT levels greater than 5 times the upper limit of normal should raise the suspition of AIH and PSC overlap syndrome. Hyperbilirubinemia is uncommon at disease onset and slightly higher IgG levels can be observed in $60 \%$ of the cases ${ }^{(76)}$.

Autoantibodies may be present, particularly in lowtiters. They are not important of the diagnosis of PSC as well as for the diagnosis of AIH and PSC overlap syndrome. Atypical perinuclear antineutrophil cytoplasmatic antiboby (pANCA) is often present, but it not specific for either PSC or IBD ${ }^{(75)}$.

Secondary causes of sclerosing cholangitis should be ruled out, particulary those associated with previous surgery, intra-arterial chemotherapy, recurrent bacterial cholangitis, intrahepatic lithiasis or common bile duct stones. Other disorders that may resemble PSC include IgG4 disease, portal biliopathy and HIV-associated colangiopathy ${ }^{(77)}$.

In subjects with clear-cut cholangiographic criteria for sclerosing cholangitis, the presence of intrahepatic lithiasis or choledocolithiasis is not sufficient to exclude PSC. In this respect, it should be emphasized that sludge and biliary stones can also aggravate the clinical course of the disease due to biliary stasis. Other clues for the diagnosis of PSC are important in this setting, including its clinical and cholangiographic features as well as the presence of concurrent IBD.

Patients with clinical, laboratory and histological features of PSC with a normal cholangiogram may harbor small-duct PSC. Differential diagnosis of this condition from other causes of intrahepatic cholestasis may be challenging. Concurrent IBD as well as exclusion of secondary causes of sclerosing cholangitis usually favor the diagnosis of small-duct PSC(75).

Histological findings can support the diagnosis of PSC, but they are usually inespecific in the early stages of the disease. Periductular obliterative fibrosis, also known as pericholangitis or onionskin lesion is a characteristic feature of PSC or small-duct PSC, but can be rarely seen also in cases of secondary sclerosing cholangitis ${ }^{(78)}$.

Liver biopsy is always needed to stablish the diagnosis of small-duct PSC, but is usually not indicated in subjects with full-blown PSC findings at MRCP or ERC, unless there is a clinical suspicion of AIH and PSC overlap syndrome ${ }^{(75)}$.

In subjects with end-stage liver disease, cholangiograms with distortion and or rarefaction of biliary tree branches are usually seen, raising the suspicion for PSC. The absence of IBD as well as typical findings of PSC, particularly in the extrahepatic bile ducts may help to exclude the disease in this setting.

\section{Recommendations}

- Patients with cholestasis of unknown cause, particularly in the absence of antimithocondrial antibody (AMA) should be submitted to MRCP to rule out PSC (Class Ia).

- Liver biopsy should be considered in those subjects with normal MRCP under suspicion of small-duct PSC. Histology is not required for diagnosis of patients with large-duct PSC by MRCP. However, it may de needed to assess the presence of PSC with features of $\mathrm{AIH}$, in those subjects with disproportionally higher aminotransferases levels more than 5 times the upper limit of normal (Class Ib).

- Colonoscopy is recommended for patients with PSC irrespective of the presence of symptoms. Multiple biopsies are recommend even if the endoscopic appearance of the colonic mucosa is normal (Class Ia).

- Patients with the diagnosis of concurrent IBD should be submitted to colonoscopic screening for colorectal neoplasia (Class Ib).

\section{Pharmacological treatment of PSC}

There is no pharmacological treatment options that have proven to be able to modify the natural history of PSC. However, ursodeoxycholic acid (UDCA), an epimer of chenodeoxycholic acid, has been widely used for the management of patients with for more than 30 years $^{(79)}$. Potential mechanisms of action of UDCA in cholestatic liver diseases involve: 1) increased hydrophilicity of the circulating bile acid (BA) pool; 2) stimulation of hepatobiliary secretion of BA, organic and inorganic anions and adenosine triphosphate (ATP); 3) reduction of BA-induced 
and cytokine-induced cytotoxicity against hepatocytes and colangiocytes; and 4) immunomodulatory and antiinflammatory effects ${ }^{(80)}$. It is important to emphasize that the relative contribution of each of these mechanisms for the anticholestatic effect of UDCA is unknown. In the context of the PSC, UDCA has been evaluated at low doses (10 to $15 \mathrm{mg} / \mathrm{kg} /$ day), intermediate doses ( 17 to $23 \mathrm{mg} / \mathrm{kg} /$ day) and high doses ( 25 to $30 \mathrm{mg} / \mathrm{kg} /$ day), in several clinical studies, which have been compiled in three meta-analyzes. ${ }^{(81-83)}$. Besides having different doses and designs and high risk of bias, these studies used different response criteria and, in general, included large numbers of patients with advanced disease, making it difficult to get definitive conclusions about the efficacy and safety of UDCA in PSC. However, despite the abovementioned methodology limitations and until new evidence is generated in additional studies, we can conclude that: a) low-dose UDCA may result in clinical and biochemical improvement, but without increase in survival; b) high doses can have a negative impact on the evolution of the disease, even in patients with early disease; c) intermediate doses can induce biochemical and histological response, without drug-related serious adverse events, but with uncertain impact on survival.

PSC carriers under treatment with UDCA should be periodically monitored with clinical examination and routine liver tests, with two main objectives: to assess response to therapy and to identify potential disease progression. Recently, three studies have identified clinical improvement in patients with PSC that showed a significant reduction in serum levels of ALP, defined as normalization of ALP or reduction to levels below 1.5 time the ULN at any time during follow-up or reduction $\geq 40 \%$ after one year of treatment with $\mathrm{UDCA}^{(84-86)}$. Although these criteria need prospective validation, they may be useful for prognostic purposes. However, it is important to emphasize that late responses can occur (even after two years of therapy) and that the suspension of UDCA may lead to significant clinical and laboratory worsening ${ }^{(79,87)}$. Thus there is no evidence that UDCA should be stopped in the absence of significant biochemical response, except in cases of suspected UDCA-related disease progression. Worsening of pruritus, fatigue and/or deterioration of hepatic synthesis tests, progressive elevation of serum ALP, and development, increase or rupture of esophagogastric varices are signs of disease progression.

Currently, there are no pharmacological alternatives for the specific treatment of the PSC. Antifibrotic agents (colchicine, penicillamine, silymarin, etc.), antimicrobial (vancomycin, minocycline, metronidazole), immunobiological agents (infliximab, etanercept) and immunosuppressants (prednisone, prednisolone, budesonide, azathioprine, methotrexate, tacrolimus, cyclosporin, mycophenolate) have not been effective and/or safe for the treatment of PSC, and are not recommended ${ }^{(75)}$. Examples of promising drugs being evaluated in ongoing clinical trials: 24-norursodeoxycholic acid (an UDCA homologous; trial identified as NCT01755507 on the ClinicalTrials.gov website), docosahexaenoic acid (fatty acid omega-3 type; NCT00325013), obeticholic acid (farnesoid X receptor agonist; NCT02177136), BTT1023 (antibody anti-VAP-1; NCT02239211) and simtuzumab (humanized monoclonal antibody against the enzyme lysyl oxidase-like 2 [LOXL2]; NCT01672853).

Bezafibrate has been successfully used as a pharmacological alternative in the PSC in a few cases reported in the literature, so that there is insufficient evidence to recommend its use $\mathrm{e}^{(88,89)}$

Corticosteroids and other immunosuppressive agents are not recommended for the treatment of PSC, except in cases of PSC with features resembling AIH, the so called "AIH/ PSC overlap syndrome" ${ }^{(68)}$ In this context, the combined regimen with prednisone and azathioprine is indicated, typically in association with UDCA. Immunosuppression (corticosteroids alone or in combination with azathioprine) is also recommended for the treatment of PSC associated to $\mathrm{IgG} 4^{(68)}$. In this case, the treatment has a minimum of three months of duration and can require maintenance therapy if there is recurrence or incomplete answer.

The most robust randomized controlled trials ${ }^{(90,91)}$ and two meta-analyzes concluded that there is no significant impact of the use of UDCA on the incidence of $\mathrm{CC}^{(81,82)}$. There is no evidence that the use of UDCA reduces the risk of development of gallbladder cancer in patients with PSC. As for colorectal cancer (CRC), the real impact of the use of UDCA remains unclear, with two studies suggesting a protective effect ${ }^{(92,93)}$, four studies noting no impact ${ }^{(94-97)}$ and one study indicating increased risk of CRC in UDCA users $^{(98)}$. Recent meta-analysis found no association between the use of UDCA and the risk of CRC or dysplasia in adults with PSC and IBD ${ }^{(99)}$. Reflecting the controversy, while the American guidelines do not recommend the use of UDCA as a prophylaxis agent against $\mathrm{CRC}^{(75)}$, the European guidelines suggest its use for high-risk patients (those with extensive colitis, positive family history or previous CRC), although it recognizes that there is limited evidence ${ }^{(68)}$.

In general, fertility is not affected and pregnancy is well tolerated in individuals with well-controlled PSC ${ }^{(100-102)}$. The presence of PSC seems to be associated with the occurrence of preterm birth, with possible contribution of the presence of IBD activity ${ }^{(101,102)}$. There is no increased risk of congenital malformations or other adverse pregnancy outcomes ${ }^{(101,102)}$. UDCA, qualified as a Class B drug by the FDA (Food and Drug Administration, USA), appears to be safe to be used in the second or third trimesters of pregnancy ${ }^{(101)}$. Even its early use during the first trimester has not been associated with negative effects on the mother or the fetus ${ }^{(101)}$. Exacerbation of PSC, worsening pruritus or de novo pruritus may occur during pregnancy or postpartum, requiring UDCA beginning or increasing dosage, and can lead to preterm birth $^{(100-103)}$. In one study, pregnant women on UDCA most frequently had stable liver tests, compared to those who did not use the drug ${ }^{(101)}$. The UDCA is excreted in breast milk in small quantities and its use was not associated with side effects in neonates ${ }^{(104,105)}$. 


\section{Recommendations}

- After detailed discussion of risks and benefits of therapy and about the limitations of available data, the use of UDCA in intermediate doses $(17-23 \mathrm{mg} / \mathrm{kg} /$ day $)$ should be considered for adult patients with PSC. (Class IIb)

- PSC carriers under treatment with UDCA should be regularly monitored with clinical examination and liver tests, to assess response to therapy and to identify possible disease progression. (Class I)

- In patients treated with UDCA, normalization or significant reduction of serum levels of ALP suggests better prognosis (Class II). There is no evidence that UDCA should be discontinued in the absence of response, except when the progression of the disease is possibly related to UDCA itself (Class II).

- There is no sufficient evidence to recommend the use of fibrates or other pharmacological alternatives as specific therapies for PSC (Class IIb).

- Immunosuppression with corticosteroids alone or in combination with azathioprine is recommended in cases of PSC with AIH-like characteristics and for the treatment of PSC associated IgG4 (Class I).

- There is no evidence that the use of UDCA reduces the risk of developing $\mathrm{CC}$ or gallbladder cancer in patients with PSC (Class III). The data available in the literature does not allow to conclude whether the use of UDCA is associated with lower risk of colorectal cancer (Class IIb).

- Pregnancy is generally well tolerated in women with compensated PSC, but there seems to be an increased risk of preterm birth (Class II). The use of UDCA can be considered during pregnancy, preferably after the first quarter (Class II).

\section{Endoscopic treatment of PSC}

Endoscopic treatment of PSC should be considered for subjects with symptomatic dominant strictures. They are considered in the presence of a stenosis with a diameter of at least 1,5 and $1 \mathrm{~cm}$, respectively, in the common bile duct (CBD) and in the hepatic duct. They usually occur in the hepatic hilum, hepatic duct or the CBD. Dominant strictures are observed in $45 \%$ to $58 \%$ of the patients with $\mathrm{PSC}^{(107)}$. Their clinical presentation is also variable, but increasing liver enzymes, jaundice and recurrent cholangitis are common features, particularly in those subjects with dominant strictures in the CBD. The presence of dominant strictures is associated with progression of PSC and their treatment by ERC may improve survival. However, it should be noted that clinical deterioration has been also reported in subjects with end-stage PSC submitted to ERC.

Endoscopic treatment of dominant strictures are associated with clinical and biochemical improvement in around $80 \%$ PSC subjects without cirrhosis ${ }^{(108)}$. However, it should not be used as a bridge to delay LT in those patients with end-stage PSC, because LT is regarded as the only treatment modality that can really alter the natural history of the disease.
The main complications of ERC in patients with PSC are pancreatitis, cholangitis, hemorrhage, perforation, worsening of cholestasis and progressive clinical and biochemical deterioration. They are more commonly observed in subjects submitted to therapeutic ERC. Pancreatitis and cholangitis after ERC are reported to occur in, respectively, $5 \%-7 \%$ and $1 \%$ of the treated patients ${ }^{(109)}$. Before endoscopic treatment, it is usually recommended antibiotic prophylaxis to prevent ascending cholangitis ${ }^{(3)}$, that is more frequently seen in those subjects with cirrhosis, concurrent AIH and CD. The level of expertise of the examiner, performance of sphincterotomy and bile duct dilatation are also associated with cholangitis ${ }^{(110)}$.

In PSC subjects without dominant strictures at disease onset, the occurence of de novo symptoms, particularly of jaundice, or clinical deterioration may suggest the development of dominant strictures.

Irrespective of the presence of those symptoms, colhangiocarcinoma have to be excluded in all subjects with PSC with dominant strictures. The screening and diagnosis of $\mathrm{CC}$ are highlighted elsewhere in this manuscript.

Most of the data concerning endoscopic treatment of PSC are not derived from RCT. Treatment options include bile duct stenting with or without previos dilation or only dilation. In one retrospective study, either dilation with or without stenting were associated with adequate biliary drainage as well as clinical improvement of patients with PSC ${ }^{(111)}$. However, a higher incidence of complication were noted in those patients who required stents, when compared to their counterparts treated only with dilation.

If stents are required, they should be changed every two to three months in order to prevent cholangitis due to stent obstruction. Some patients may require prolonged use of stent for 12 months to ensure resolution of those dominant strictures. The main disadvantage of stents are their associated risk for obstruction (usually seen in half of those treated patients), that may evolve to cholangitis and sepsis. Use of stents for a short period of time (mean of 11 days) was advocated in some reports to be associated with better outcomes ${ }^{(112)}$. There is no RCT published until now, comparing dilation vs. stents in the management of dominant strictures in PSC.

\section{Recommendations}

- Endoscopic treatment can be indicated in centers with expertise in therapeutic ERC in subjects with PSC with dominant strictures (Class IIb)

- Before endoscopic treatment, it is mandatory to excluse cholangiocarcinoma (Class I)

- Endoscopic treatment can be performed with bile duct dilation or stenting due to the lack of evidence-based data to support either one of those treatment modalities (Class IIb)

\section{Diagnositc and therapeutic implications for PSC in children}

Primary sclerosing cholangitis is uncommon in children, being five times less frequent when compared to adults. The 
incidence of PSC in children have been stimated as 0,23 cases for 100.000 person-years. There are only 250 cases of PSC in children published in the literature from different referral centers, which hamper the development of evidence-based guidelines about the management of PSC in this age-group ${ }^{(75,113)}$.

Most of the clinical findings of the disease in children are identical to those observed in adults as well as in other children with cholesttic liver diseases. Aproximately $20 \%$ of the children are asymptomatic. Some may present clinical features related to childhood such as growth retardation and puberty delay ${ }^{(115)}$. The frequency of AIH and PSC overlap is more frequently reported in children, when compared to adults. CC is rarely seen and $\mathrm{IgG} 4$ disease have not been reported in childhood. Some inherited cholestatic diseases of childhood may resemble PSC. In this regard, mutations of $\mathrm{ABCB} 4$ gene, related to progressive familiar intrahepatic cholestasis type 3 , have been reported in children with smallduct PSC(75,113,114,116)

The diagnosis of PSC in children is based on the presence of clinical, laboratory and cholangiographic features of the disease, after exclusion of secondary causes of sclerosing cholangitis. It should be pointed out that ALP is not regarded as a marker of cholestasis in childhood, due to its physiological increase during the bone growth, seen in this age-period. GGT, on the other hand, is regarded as a reliable tool to assess cholestasis in children. In this regard, $97 \%$ of those children with PSC had abnormal GGT at diagnosis ${ }^{(75,113-115)}$. The ratio of GGT/AST is useful to detect AIH and PSC overlap in children ${ }^{(114)}$. When compared to adults, children tend to have lower levels of bilirubin and higher leves of AST and ALT, due to the lower frequency of dominant strictures and $\mathrm{CC}$ and higher frequency of concurrent AIH usually seen in pediatric PSC $^{(75,113-117)}$.

Liver biopsy is important in childhood PSC, particularly to assess small-duct PSC in subjects with normal cholangiograms or other immune-mediated diseases. It is also crucial for the diagnosis of AIH and PSC overlap ${ }^{(75,113-118)}$.

Magnetic resonance cholangiography is the procedure of choice for the diagnosis of PSC, and exhibit the same changes previously describe for adults. Aproximately $40 \%$ of the patients with normal cholangiograms may have small-duct PSC at liver biopsy ${ }^{(75,113-115)}$.

$\mathrm{CC}$ is very rare in children with PSC ${ }^{(75,114,118)}$. The younger patient with PSC and CC reported in the literature had 14 years of age and longstanding $U C^{(114)}$.

The association of PSC with IBD in children ranges from $33 \%$ to $81 \%$. Therefore, colonoscopy is usually recommended even in children without bowel symptoms. If the initial colonoscopy disclose normal findings, subsequent examinations should be performed only if the patient develop typical symptoms of IBD. Even in those subjects with concurrent IBD, screening of CRC is controversial, since it s rarely seen before 16 years of age ${ }^{(75,113,114)}$.

Up to now, there is controversy on the employment of UDCA in adults with PSC and there are no controlled data on their use in children ${ }^{(75,113-115,117,119)}$ Antibiotics, such as oral vancomycin are still under investigation ${ }^{(114-116,120)}$.
Dominant strictures are less common in children with PSC, but are also amenable to treatment, as highlight previously $^{(114,115)}$.

\section{Recommendations}

- Due to the scarcity of cases of PSC in children, it is not possible to stablish evidence-based recommendations for the management of the disease in the pediatric age group (Class I)

- Clinical manifestations are similar to those observed in adults. Dominant strictures and CC is rarely seen. On the contrary, AIH and PSC overlap is much more common (Class IIa)

- MRCP is the procedure of choice for diagnosis of PSC in children. Liver biopsy is usually necessary to rule out other common causes of secondary sclerosing cholangitis (Class IIa)

- Colonoscopy should be performed to assess concurrent IBD (Class IIa)

- There is scarcity of data concerning treatment options for PSC in children (Class I)

\section{Part III: PRIMARY BILIARY CIRRHOSIS}

\section{Diagnosis}

PBC is now diagnosed earlier in its clinical course, with more than half of the patients being asymptomatic at the time of diagnosis. Among symptomatic patients, fatigue and pruritus are the most common symptoms, present in up to $70 \%$ of the cases. The pathogenesis of fatigue is unknown and appears to be multifactorial, with autonomic dysfunction playing a significant role. Other causes of fatigue, including anemia, hypothyroidism, adrenal insufficiency, depression, and sleep disorder must be excluded prior to attributing the symptom to PBC. Patients complain of daytime sleepiness and lack of energy. Despite its impact in the quality of life, no correlation could be demonstrated so far between fatigue and severity of liver disease. Indeed, fatigue does not improve with treatment of the disease. Pruritus, on the other hand, is a more specific symptom. It can be local or diffuse, usually worse at night, and often extremely debilitating. The onset of pruritus typically precedes the onset of jaundice by months to years, and in fact it tends to improve or disappear as the disease progresses. Occasionally, itching starts during the third trimester of pregnancy and persists after delivery, in contrast to the itching associated with intrahepatic cholestasis of pregnancy, which resolves after delivery. The pathogenesis of pruritus in PBC is also unclear and several hypotheses have been formulated, including derangements in bile salt production and excretion, change in progesterone metabolites, histamine imbalance and increased levels of endogenous opioids. Recent clinical and experimental evidence highly suggest that the neurotransmitter lysophosphatidic acid is an important mediator of the cholestatic itching ${ }^{(68,121)}$.

The median survival for symptomatic patients ranges from 5 to 8 years from the onset of disease. In addition, 
nearly $25 \%$ of asymptomatic patients at the time of diagnosis progress to liver failure within 10 years $^{(134)}$. Contemporary series have shown that with earlier diagnosis, $42-66 \%$ of asymptomatic patients are at an earlier histological stage (stages I and II), whereas $82 \%$ of symptomatic patients are at a later histological stage of disease (stages III and IV). Patients who are symptomatic at presentation appear to have lower rates of biochemical response to UDCA and an increased risk of progression to cirrhosis and its complications $^{(135)}$.

Among the most common extra-hepatic complications of $\mathrm{PBC}$ are hyperlipidemia, cutaneous hyperpigmentation and osteoporosis. Patients who have advanced stage $\mathrm{PBC}$ have a five-fold increase in risk for developing osteoporosis compared with those who have early stage.

Anti-mitochondrial antibody (AMA) negative patients have similar clinical course, response to therapy and complication rate compared to their AMA positive counterparts. ANAs are detected in $30-50 \%$ of patients with PBC, and these may display patterns that are $95 \%$ specific for PBC. Presence of antibodies against the nuclear pore protein gp210, with rim-like membranous pattern on IF, have been associated with more severe interface hepatitis, lobular inflammation and ductopenia, translating into worse clinical course and increased rates of progression towards liver failure ${ }^{(136)}$. On the other hand, presence of anticentromere antibodies (ACA), noted in up to $30 \%$ of patients with PBC, may be a marker of future development of systemic sclerosis ${ }^{(137)}$. In addition, ACA positivity has been associated with development of portal hypertension, possibly due to severe ductular reaction ${ }^{(136)}$.

Associations between $\mathrm{PBC}$ and other autoimmune diseases have been consistently reported and reinforce the current classification of $\mathrm{PBC}$ as an autoimmune condition. The reported prevalence of an overlap syndrome of $\mathrm{PBC}$ and AIH ranges between 2 and $20 \%{ }^{(138)}$. Overlap syndrome PBC-AIH is discussed in details in the corresponding topic.

Sicca syndrome is seen in $60-80 \%$ of patients, and should be routinely investigated in those complaining of dry eyes/ dry mouth. Tests for dry eyes include Schirmer and ocular surface staining (Rose Bengal). Hashimoto's thyroiditis is present in $20 \%$ of patients with PBC, often predating its diagnosis. Scleroderma is a multisystem autoimmune rheumatic disorder present in up to $8 \%$ of patients with PBC. The frequency of Raynaud's phenomenon is four-fold higher in $\mathrm{PBC}$ when compared to the general population. In turn, up to $25 \%$ of patients with scleroderma are AMA positive. The prevalence of rheumatoid arthritis in $\mathrm{PBC}$ has been reported between $1.8 \%$ and $5.6 \%$, whereas the prevalence of AMA positivity in patients with rheumatoid arthritis has been estimated at $18 \%$. The association between $\mathrm{PBC}$ and polymyalgia has been described mainly in single case reports, but lupus erythematosus occurs in $2.7 \%$ to $7.5 \%$ of PBC cases. Associations with Addison disease and autoimmune diabetes are uncommon. Among the gastrointestinal disorders, celiac disease has been the most studied. Celiac disease is present in $6 \%$ of patients with PBC; in contrast, $3 \%$ of celiac patients were found to have PBC. Serological screening testing for celiac disease is not routinely recommended in $\mathrm{PBC}$, unless there is a strong suspicion for the disease ${ }^{(139)}$

Multiple lines of evidence support the concept of genetic predisposition in $\mathrm{PBC}$, including 1) a high concordance rate among monozygotic twins $(63 \%), 2)$ an increased prevalence of other autoimmune conditions in patients with $\mathrm{PBC}$ and their family members, 3) existence of familial clusters of PBC and 4) an increased rate of AMA positivity among first degree relatives of patients with $\mathrm{PBC}^{(140-142)}$. Despite the fact that its benefit hasn't yet been demonstrated, we recommend screening adult first degree relatives of patients with PBC with measurement of serum ALP level, and testing for AMA if ALP is elevated.

Data from Brazil are scarce. Between 1995 and 2013, 246 patients referred to the Autoimmune and Cholestasis Diseases Group at the University of São Paulo were diagnosed with PBC. Table 3 illustrates the relevant clinical presentation and serology data for these patients.

TABLE 3 - Clinical and laboratory data on 246 patients diagnosed with PBC at HC-FMUSP (personal communication, data not published)

\begin{tabular}{ll} 
Cases at HC-FMUSP $(\mathbf{N}-246)$ & $\mathbf{n}(\%)$ \\
\hline Initial clinical presentation & \\
Asymptomatic & $101(41)$ \\
Pruritus & $83(33.7)$ \\
Fatigue & $52(21)$ \\
Portal hypertension & $29(11.7)$ \\
& \\
Serology & \\
AMA (+) & $233(94.7)$ \\
Isolated ANA (+) & $13(5.2)$ \\
Anti-gp210 (+) & $46 / 159(28.9)$ \\
Anti-sp100 (+) & $44 / 159(27.6)$ \\
Anti-centromere antibody $(+)$ & $39 / 159(24.5)$ \\
IgM >2 X ULN & $122(49.5)$
\end{tabular}

Associations with autoimmune diseases

$\begin{array}{ll}\text { Sicca syndrome } & 40(16.2) \\ \text { Hypothyroidism } & 29(11.7) \\ \text { Scleroderma } & 21(8.5) \\ \text { Raynaud's } & 20(8.1) \\ \text { Psoriasis } & 7(2.8) \\ \text { Autoimmune hepatitis } & 6(2.4) \\ \text { Celiac disease } & 5(2.1) \\ \text { Idiopathic thrombocytopenic purpura } & 4(1.6) \\ \text { Vitiligo } & 3(1.2) \\ \text { Polymyositis } & 3(1.2) \\ \text { Mixed connective tissue disorder } & 2(0.8)\end{array}$

AMA: antimithocondrial antibody; ANA: antinuclear antibody; ULN: upper limit of normal 
While the incidence of PBC appears to be increasing in recent years, most cases are diagnosed in asymptomatic stages, likely due to increased awareness, improved diagnostic tools, and improved access to routine biochemical tests. Once cholestasis is identified, typically by means of elevated serum ALP, an initial evaluation is triggered to confirm the diagnosis of PBC, as shown in Figure 1. It is important to exclude other etiologies such as drug-induced cholestasis, biliary obstruction, granulomatous hepatitis, autoimmune hepatitis and PSC, including small duct PSC.

According to the AASLD, the diagnosis of PBC is made when 2 of the following 3 criteria are met: 1) Biochemical cholestasis with predominance of ALP, 2) presence of AMA and 3) histological evidence of non-suppurative destructive cholangitis ${ }^{(121)}$.

AMA should be tested initially by II in rodent tissue and HEp-2 cells, as these are available and standardized tests in our country ${ }^{(122)}$. This marker is positive in $90-95 \%$ of patients. Detection of AMA positivity in patients without clinical or laboratory evidence of PBC may indicate a high probability of $\mathrm{PBC}$ being diagnosed within the next 10 years. AMA titers below 1:80 may be present in the normal population $(<1 \%)$, and therefore should be interpreted with some caution ${ }^{(122,123)}$. Low titer AMA can be detected in other conditions, such as lupus, antiphospholipid syndrome, autoimmune hemolytic anemia, alcoholic liver disease and hepatitis $\mathrm{C}^{(4)}$. If necessary, ELISA or immunoblotting should be used as confirmation methods for detection of M2 antibodies (against the 2-oxoacid dehydrogenase complex), considered PBC-specific, although its positivity has already been described in other diseases, such as hepatitis $\mathrm{C}$ and autoimmune hepatitis ${ }^{(125,126)}$, and even in normal individuals, in low titers ${ }^{(126)}$. Testing for antiM2 is particularly useful in AMA-negative PBC patients; ELISA or immunoblotting for gp210 and sp100 can also be very useful but must be done in specialty labs given the cost and expertise involved.

In addition to AMA, ANA can be very useful for diagnosis. ANAs are present in $30-50 \%$ of $\mathrm{PBC}$ cases. The reticular speckled cytoplasmic pattern, for instance, typically correlates with presence of AMA, and should trigger further AMA testing if this hasn't been done. Furthermore, certain ANA patters are highly specific for PBC. These are (a) the rim-like membranous pattern, targeting gp 210 and p62, and (b) the multiple nuclear dots pattern, targeting several proteins including sp 100 and protein promielocytic. The anti-centromere, although not specific, is usually described in $\mathrm{PBC}^{(127)}$. This pattern can also be seen in patients with scleroderma, Sjogren's or with isolated Raynaud's phenomenon. Thus, the ANA pattern can both aid detection of AMA and assist in the diagnosis of AMA negative PBC cases. Moreover, these autoantibodies can provide prognostic information, since presence of anti-gp210 and anti-

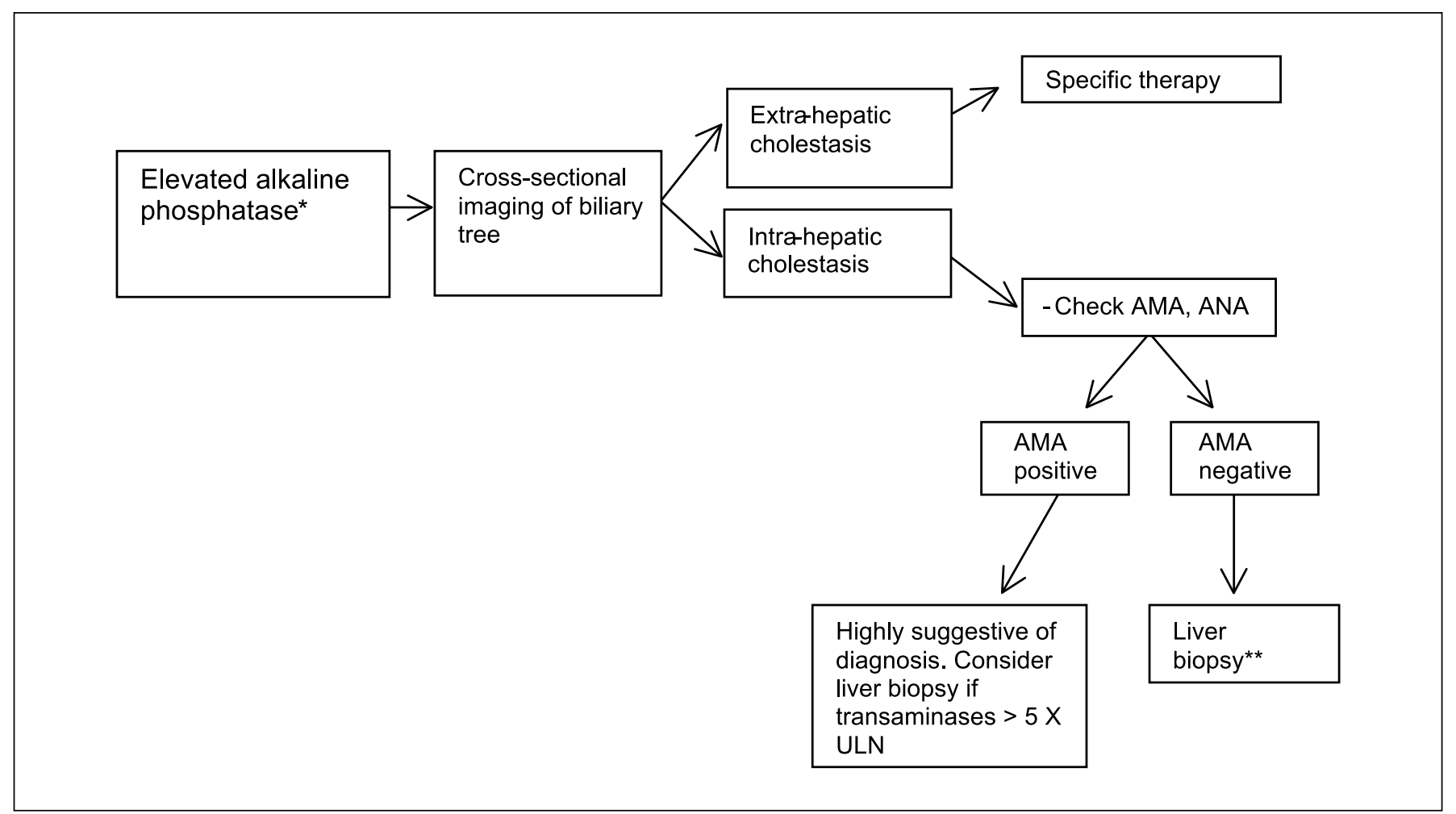

FIGURE 1. Suggested algorithm to evaluate patients with laboratory evidence of cholestasis. AMA - anti-mitochondrial antibody, ANA anti-nuclear antibody; ULN -upper limit of normal

* Pay attention to different cut-off values for special populations, such as children, elderly and pregnant women

**Liver biopsy is not indicated for AMA negative cases when the ANA is positive with a rim-like or multiple nuclear dots pattern. 
centromere has been associated with a more rapid progression to liver failure and portal hypertension ${ }^{(123,128)}$.

Non-suppurative destructive cholangitis affecting the interlobular and septal bile ducts is typical and one of the diagnostic criteria for PBC. This inflammatory infiltrate consists mostly of lymphocytes, plasma cells, eosinophils and macrophages, sometimes with epithelioid granulomas. When this focal biliary injury is marked, the term florid duct lesion is used. Albeit useful for diagnostic and prognostic purposes, liver biopsy is not required for the diagnosis of PBC. The biopsy is formally recommended in all AMA-negative patients and whenever an alternative or concomitant disease must be excluded, such as when the diagnosis of overlap syndrome with autoimmune hepatitis comes into question.

Histology classifies PBC into 4 stages. Stage I is characterized by portal inflammation, with or without florid duct lesion. In stage II, the inflammatory infiltrate extends to the periportal areas, with evidence of interface hepatitis. In stage III there is architectural distortion, with development of multiple fibrous septa. Cirrhosis, with regenerating nodule formation, characterizes stage IV. However, histology is not necessarily required to estimate fibrosis stage and several non-invasive methods are emerging to estimate fibrosis stage and prognosis.

Non-invasive methods to estimate fibrosis and predict prognosis are promising. A recent study sought to identify prognostic variables among 386 patients with $\mathrm{PBC}$ in the $\mathrm{UK}^{(129)}$. In this study, an AST/platelet ratio index (APRI) greater than 0.54 was predictive of survival free of LT, independent of response to UDCA treatment. Transient elastography (Fibroscanä) is among the best-studied and most accurate non-invasive methods to predict fibrosis in PBC. In a large comparative study involving 150 patients with $\mathrm{PBC}$, transient elastography performed better than the biochemical surrogates, such as APRI, FIB04, hyaluronic acid, AST/ALT ratio and Mayo risk score in predicting significant fibrosis, severe fibrosis and cirrhosis, with a cut-off of $16.9 \mathrm{kPa}^{(130)}$. Further, worsening of liver stiffness in serial examinations during the follow-up period correlated with a greater frequency of clinical decompensation, death or liver transplant. The diagnostic performance of acoustic radiation force impulse (ARFI) elastography was also evaluated in 61 patients with $\mathrm{PBC}$ and correlated with histological stage. The areas under the ROC curve were $0.83,0.93$ and 0.9 , for stages ${ }^{(3)}$ II, II and IV, respectively. The optimal cutoff values for the shear wave velocity were $1.51 \mathrm{~m} / \mathrm{s}, 1.79$ $\mathrm{m} / \mathrm{s}$ and $2.01 \mathrm{~m} / \mathrm{s}$, respectively, for stages ${ }^{(3)}$ II, II and IV ${ }^{(131)}$.

Since esophageal varices may develop rather early in PBC due to non-cirrhotic portal hypertension in the setting of nodular regenerative hyperplasia, the Newcastle index has been proposed as a prognostic tool, with AUROC 0.86 for detection of varices ${ }^{(132)}$. By using the score one would hope to avoid unnecessary endoscopy. This index only requires routine laboratory tests - albumin, ALP and platelets - and may be calculated using the link http://www.uk-pbc.com/media/ sites/researchwebsites/uk-pbc/pbc130328.html. Similarly, the Mayo risk score can be calculated from their website and a score $\geq 4.5$ indicates the need for endoscopy ${ }^{(133)}$.

\section{Recommendations}

- AASLD criteria should be adopted for initial evaluation of PBC patients (Class I).

- The diagnosis of PBC is established when 2 of the following criteria are met:

- Chronic elevation of ALP

- Presence of AMA

- Liver biopsy demonstrating non-suppurative destructive cholangitis and destruction of interlobular bile ducts

- Regarding autoantibodies:

- AMA titers $\geq 1: 80$ are considered significant (Class I)

- Anti-M2 antibodies should be ordered if AMA is negative, the titer of AMA is $<1: 80$ or the pattern is not typical (Class I)

- Testing for ANA and pattern characterization by indirect immunofluorescence in HEp-2 cells or by immunoblotting and ELISA must be requested in AMA negative patients, to assess for PBC-specific ANAs (Class IIa)

- Liver biopsy is recommended in AMA-negative patients and/or when associated liver disease is suspected (Class I).

- Non-invasive methods for staging are under investigation and cannot be routinely recommended (Class IIb)

\section{Treatment with ursodeoxycholic acid}

Ursodeoxycholic acid (UDCA), the 7b epimer of chenodeoxycholic acid, is a hydrophilic bile acid that has been used for decades in the treatment of PBC. Its beneficial effects are explained by cytoprotective, choleretic, immunomodulatory and anti-inflammatory properties that have been demonstrated in clinical and experimental studies. Currently, both the AASLD and the European Association for the Study of the Liver (EASL) endorse the use of UDCA for the treatment of PBC with elevated ALP, independent of the disease stage ${ }^{(68,121)}$. This recommendation is based on several placebo-controlled, meta-analyzes and long-term cohort studies demonstrating beneficial effects of UDCA. The recommended dose is $13-15 \mathrm{mg} / \mathrm{kg} / \mathrm{day}$, administered as single or multiple daily doses. Adverse events are uncommon, with mild gastrointestinal intolerance and minimal weight gain reported in the first year of treatment.

Studies conducted in the pre-UDCA era have demonstrated overall survival free of LT of approximately 10-15 years from the time of diagnosis, clearly decreased when compared to the general age - and gender-matched population. Treatment with UDCA has been shown to significantly lower the serum levels of bilirubin, ALP, GGT, IgM and total cholesterol ${ }^{(143,144)}$. Studies also demonstrated that treatment with UDCA slows histological progression, delays development of esophageal varices, and normalizes survival free of LT for patients who begin treatment at earlier histological stages ${ }^{(145-148)}$. Unfortunately, UDCA does not improve fatigue or pruritus. A combined analysis of the three largest placebo-controlled studies following patients for up to 4 years noted reduction in the number of deaths and increase in survival free of LT 
in the treated group ${ }^{(145)}$. In this study, only patients with advanced disease, characterized by total bilirubin $>1.4$ $\mathrm{mg} / \mathrm{dL}$, benefited from UDCA treatment. However, given the known protracted clinical course of early stage PBC, a much longer follow-up would be necessary to demonstrate survival benefit in this population. Since then, numerous observational studies from Europe and North America have shown that treatment with UDCA is associated with excellent survival free of LT, especially patients with early histological stage and who have biochemical response to $\mathrm{UDCA}^{(143,148-151)}$. The decline in number of patients receiving liver transplant for $\mathrm{PBC}$ in Europe and North America is seen as further evidence of the survival benefit provided by long-term use of UDCA in PBC ${ }^{(152,153)}$

The Cochrane meta-analyses have questioned the effect of UDCA on survival. In the most recent 2012 update, the studies included allowed investigators to conclude that UDCA improves liver biochemistries and histology. However, a survival benefit was not demonstrated ${ }^{(154)}$. The meta-analysis was limited by a high risk of bias in 15 of the 16 studies included, a relatively small number of patients in some of the studies, heterogeneity with respect to UDCA doses and inclusion of studies with sub-therapeutic doses of UDCA, as well as a mean follow-up time of 24 (3-90) months, which was insufficient to evaluate survival of a disease with median survival of $10-15$ years.

The therapeutic response to UDCA should be evaluated after 1 year of treatment by monitoring the canalicular enzymes, especially ALP ${ }^{(68,121,145)}$. Approximately $60 \%$ of patients respond to treatment with UDCA. Among those who respond, biochemical improvement will be seen by 1-6 months after initiation of therapy, with most patients showing response by 3 months. Typically, an early significant drop is noted in serum ALP, followed by a progressive, slow, continued drop. To date, several sets of criteria have been developed by multiple investigators to assess biochemical response to UDCA and predict long-term outcomes, described in Table $4^{(155,156)}$. More recently, a pooled meta-analysis by the Global PBC

\begin{tabular}{|c|c|}
\hline Criteria & Definition \\
\hline Paris I & $\begin{array}{l}\text { ALP }<3 x \text { ULN, AST }<2 x \text { ULN and bilirubin } \leq 1 \\
\mathrm{mg} / \mathrm{dl} \text { after } 1 \text { year of UDCA }\end{array}$ \\
\hline Barcelona & $\begin{array}{l}\text { ALP decline of more than } 40 \% \text { towards baseline } \\
\text { value or a normal level after } 1 \text { year of UDCA }\end{array}$ \\
\hline Rotterdam & $\begin{array}{l}\text { Normalization of bilirubin and albumin after } \\
\text { treatment with UDCA when one or both } \\
\text { parameters were abnormal before treatment, or } \\
\text { normal bilirubin or albumin after treatment when } \\
\text { both were abnormal at entry }\end{array}$ \\
\hline Paris II & $\begin{array}{l}\text { ALP and AST } \leq 1.5 \times \text { ULN and normal total } \\
\text { bilirubin after } 1 \text { year of UDCA }\end{array}$ \\
\hline Toronto & ALP $<1.67 x$ ULN after 2 years of UDCA \\
\hline
\end{tabular}

FIGURE 2. Definition of biochemical response to UDCA according to several published criteria
Study Group included data from 15 cohort studies and a total of 4845 patients, of whom 1118 reached a clinical endpoint. This meta-analysis showed excellent correlation between serum ALP and bilirubin and survival free of LT $^{(156)}$. The greater the reduction in serum ALP levels after 1 year of treatment with UDCA, the better the transplant-free survival. The authors concluded that serum ALP and bilirubin are good surrogate markers for clinically meaningful endpoints and can be used both for patient care and in clinical trials.

Regardless of the criteria utilized, about $40 \%$ of patients with PBC will have incomplete response to UDCA, or lack thereof. UDCA non-responders demonstrate persistent biochemical abnormalities and histological progression. These patients have poorer prognosis ${ }^{(147-149)}$. Ductopenia, advanced histological stages (stages III and IV) and moderate-severe interface hepatitis, bilirubin $>1 \mathrm{mg} / \mathrm{dL}$, low serum albumin levels, portal hypertension and the Mayo risk score are all pre-treatment factors associated with poorer response to UDCA and poorer overall prognosis ${ }^{(146-149,151,155)}$.

As PBC characteristically affects women older than 40 years of age, pregnancy is not common after the diagnosis. Case series of pregnant women with PBC show that in most cases the disease remained stable throughout pregnancy, although a biochemical exacerbation was often seen after delivery ${ }^{(157)}$. Use of UDCA seems to be safe during pregnancy (Category B by the US FDA) and lactation. However, since UDCA increases lithocholic acid levels, with known embryotoxicity, UDCA is best avoided during the first trimester. Although limited, available data suggest that successful pregnancies can be expected in patients with $\mathrm{PBC}$.

Many other drugs have been studied in PBC both as monotherapy and in combination with UDCA, including penicillamine, azathioprine, thalidomide, sylimarin, colchicine, methotrexate and rituximab. To date, however, there is no data to support the use of other agents. In addition, attempting higher UDCA doses is not recommended since this strategy failed to improve biochemical response.

\section{Recommendations}

- All patients with PBC and elevated serum ALP should be treated with UDCA $13-15 \mathrm{mg} / \mathrm{kg} /$ day, even if asymptomatic at presentation (Class I)

- Response to therapy should be evaluated after 1 year of treatment. This should be done by measuring ALP and bilirubin levels (Class IIa)

- If use of bile acid sequestrants is necessary for treatment of pruritus, UDCA should be administered 2-4 hours prior to or after its ingestion (Class I).

\section{Treatment of patients with inadequate response to UDCA}

When evaluating a patient with poor biochemical response to UDCA, one should inquire about compliance to therapy and exclude alternative or concomitant diagnoses, such as celiac disease, steatohepatitis and overlap syndrome with AIH. Additional serologic work-up with or without a liver biopsy may be necessary at the discretion of the Hepatologist. 
As discussed above, investigators have proposed and validated criteria defining patients with better overall transplant-free survival while on UDCA therapy (Table 4). Such biochemical response to UDCA, as well as a few noninvasive methods to estimate fibrosis, is used in the clinical management of patients with PBC. But which of the available criteria can better distinguish patients with and without good longterm prognosis? Recently, the "Global PBC Study Group" examined data from 15 North-American and European centers including 2924 patients with PBC treated with UDCA and followed for a median time of 7 years. The investigators concluded that the Rotterdam and Paris I criteria were the most robust and better predicted 10-year survival free of transplantation ${ }^{(158)}$. Nevertheless, other criteria such as Barcelona and Toronto could also predict prognosis independent of Rotterdam and Paris I.

The same group - "Global PBC Study Group" - also showed that both ALP and total bilirubin have independent prognostic value ${ }^{(156)}$. After analyzing data from 4845 patients, the investigators found that ALP $>2 \mathrm{X} \mathrm{ULN}$ after 1 year of treatment with UDCA correlated with greater frequency of clinical decompensation, death or liver transplant [HR 2.49 (2.14-2.89)]. As expected, serum bilirubin also correlated with outcomes, with total bilirubin $>1 \mathrm{mg} / \mathrm{dL}$ indicating worse survival at 5, 10 and 15 years. Importantly, this study showed that ALP and bilirubin may be used as prognostic factors independent of patient's characteristics (gender and age), histological stage [early (I\&II) vs. advanced III\&IV)], and treatment with UDCA (treated vs. untreated). Unlike ALP, total bilirubin only becomes elevated with development of hepatic dysfunction, late in the course of the disease. Serum ALP, therefore, can be used to identify patients with poorer prognosis who would benefit from adjuvant therapy.

Although patients with incomplete response to UDCA have worse survival than the general population, a Spanish study clearly demonstrated that observed survival was better than that predicted by the Mayo risk score ${ }^{(148)}$. With that in mind, UDCA should be continued at recommended doses even for patients who fail to demonstrate biochemical response. Use of penicillamine, azathioprine, sylimarin, colchicine and methotrexate is not supported by the available literature; nevertheless, use of corticosteroids such as budesonide is controversial and will be discussed further.

Budesonide is a non-halogenated glucocorticoid, absorbed in the small bowel. In healthy individuals, $90 \%$ of the orally administered dose is metabolized during its first passage through the liver. Compared to prednisolone, budesonide is 15-20 times more potent. Two randomized placebo-controlled trials compared UDCA/budesonide vs. UDCA/placebo in a total of 116 patients with $\mathrm{PBC}^{(159,160)}$. Both studies had a 2-year treatment duration. Results suggest that adding budesonide to UDCA translates into biochemical and histological improvement. Inflammation and fibrosis scores improved in the UDCA/budesonide groups and worsened in the UDCA/ placebo groups. However, this benefit appears to be restricted to patients with early histological stage (I-II). In fact, the use of budesonide in patients with stage IV PBC has been associated to development of portal vein thrombosis and is discouraged. Furthermore, prolonged used of budesonide is associated with some of the side effects seen with long term steroid use, including a decrease in bone mineral density.

Other investigational agents include the fibrates and agonists of nuclear farnesoid X receptor (FXR). Fibrates are agonists of the nuclear receptor PPAR-alpha, with antiinflammatory and choleretic properties. Several small studies have shown significant improvement in liver biochemistries and serum IgM in patients with PBC and incomplete response to UDCA who are treated with fibrates ${ }^{(161-170)}$. One meta-analysis of 6 randomized studies including 151 Japanese patients, concluded there was not enough evidence to support or refute an effect of bezafibrate on the morbi-mortality of patients with $\mathrm{PBC}^{(171)}$. Remarkably, all studies had a high risk of bias, mainly because of questions regarding the randomization and blinding methodologies. In turn, a recent meta-analysis of 6 studies using fenofibrate for 102 patients with $\mathrm{PBC}$ and incomplete response to UDCA concluded that treatment is associated with significant reduction of ALP, GGT, TB and $\mathrm{IgM}^{(172)}$. A large, randomized, placebo-controlled study is not yet available to support the use of fibrates as adjuvant therapy in PBC. Of note, marked inflammatory activity, stage IV fibrosis and severe ductopenia have been associated with lack of response ${ }^{(173)}$. Thus, like UDCA, fibrates also seem to be more beneficial in patients with early PBC (histological stages I and II). Side effects attributed to fibrates include esophageal reflux, nausea, myalgia and even hepatitis.

Obeticholic acid (6-ethyl chenodeoxycholic acid) is a highly potent synthetic derivative of chenodeoxycholic acid that selectively activates FXR. When activated, this receptor directly modulates the synthesis, metabolism and excretion of bile acids. In phase 2 studies, patients with $\mathrm{PBC}$ and incomplete response to UDCA were randomized to receive obeticholic acid versus placebo in combination with UDCA ${ }^{(174)}$. Those receiving obeticholic acid had a significant drop in serum ALP and GGT when compared to those on placebo. Other liver biochemistries and inflammatory markers also decreased significantly. Pruritus was the main adverse event and was dose-dependent.

\section{Recommendations}

- Biochemical response should be evaluated after 1 year of treatment with UDCA to assess prognosis and determine need for adjuvant therapy (Class IIa).

- There is no consensus with regards to the best criteria to determine biochemical response to UDCA. Given costs and ease to use, we suggest using a combination of total bilirubin $\leq 1 \mathrm{mg} / \mathrm{dL}$ and/or ALP $\leq 2 X$ ULN (Class IIa).

- There is no consensus with respect to treatment of patients with incomplete response to UDCA. We recommend assessing patients' compliance with therapy and considering alternative or concomitant diagnoses. A liver biopsy may be needed at the hepatologist's discretion. Budesonide may be considered in patients with PBC, stage I-II and incomplete response to UDCA, especially if there is marked inflammatory activity (Class IIb). 
- There is not enough evidence to support routine use of fibrates or FXR agonists at this time, although evidence is rapidly accumulating for the use of obeticholic acid. (Class IIb).

\section{Part IV: Overlap Syndromes of Autoimmune Dis-} eases of the Liver

Overlap syndromes of ALD are characterized by the findings of typical features of AIH and either PSC (AIH-PSC overlap) or PBC (AIH-PBC overlap) in the same patient, either at the presentation of the ALD or sequentially during its follow-up or after institution of treatment. Most of the cases described in the literature are AIH-PBC overlap in adulthood, but AIH-PSC overlap have also been described mainly in children with $\operatorname{ALD}^{(175,176)}$. Overlap syndromes can be considered as: 1) sequential presentation of two distinct ALD, 2) more than one clearly-defined disease, 3) a continuous spectrum of fluctuating clinical, laboratory and histological features of more than one ALD, 4) a clear-cut clinical entity that can be defined as AIH-PBC and AIH-PSC overlaps, 5) presence of one better-defined ALD with features of another $\mathrm{ALD}^{(68,175)}$.

The definition of overlap syndromes of ALD is cumbersome due to the absence of diagnostic criteria the could be used for their diagnosis, the presence of different synonyms employed for its designation, such as overlap, variant, mixed or marginal syndromes, as well as different terminology recently, applied to call AIH-PSC overlap in children and AIH-PBC overlap, such as autoimmune sclerosing cholangitis (ASC) and autoimmune cholangitis ${ }^{(175,176)}$. In this regard, autoimmune cholangitis was initially employed to describe patients with features of PBC lacking AMA with circulating ANA and a god response to immunossupression (IS) with either corticosteroids and/or azathioprine. It is not used anymore as it is recognized solely as a serological variant of PBC. The adoption of ASC in substitution for AIH-PSC overlap is up to now controversial and has been used only in the pediatric literature ${ }^{(8)}$. The clinical, laboratorial, histological and cholangiographic features of AIH-PBC and AUH-PSC overlaps are variable and non-standardized. It must be taken into account, before characterization of any overlap syndrome, that most of them can be occasionally seen in subjects with typical AIH, PBC and PSC (Table 4).

Pathogenesis of overlap syndromes of ALD remains unknown, but it is not recognized to be distinct to AIH, PBC and PSC. However, shared HLA-linked susceptibility was reported to occur in AIH, PSC and AIH-PSC overlap ${ }^{(24,177)}$. The impact of those overlapping features of more than one ALD in prognosis is not entirely clear, but lower response to treatment, accelerated disease progression, higher risk of treatment failure including death and requirement for

TABLE 4. Clinical, laboratory and histological features of AIH, PBC and PSC

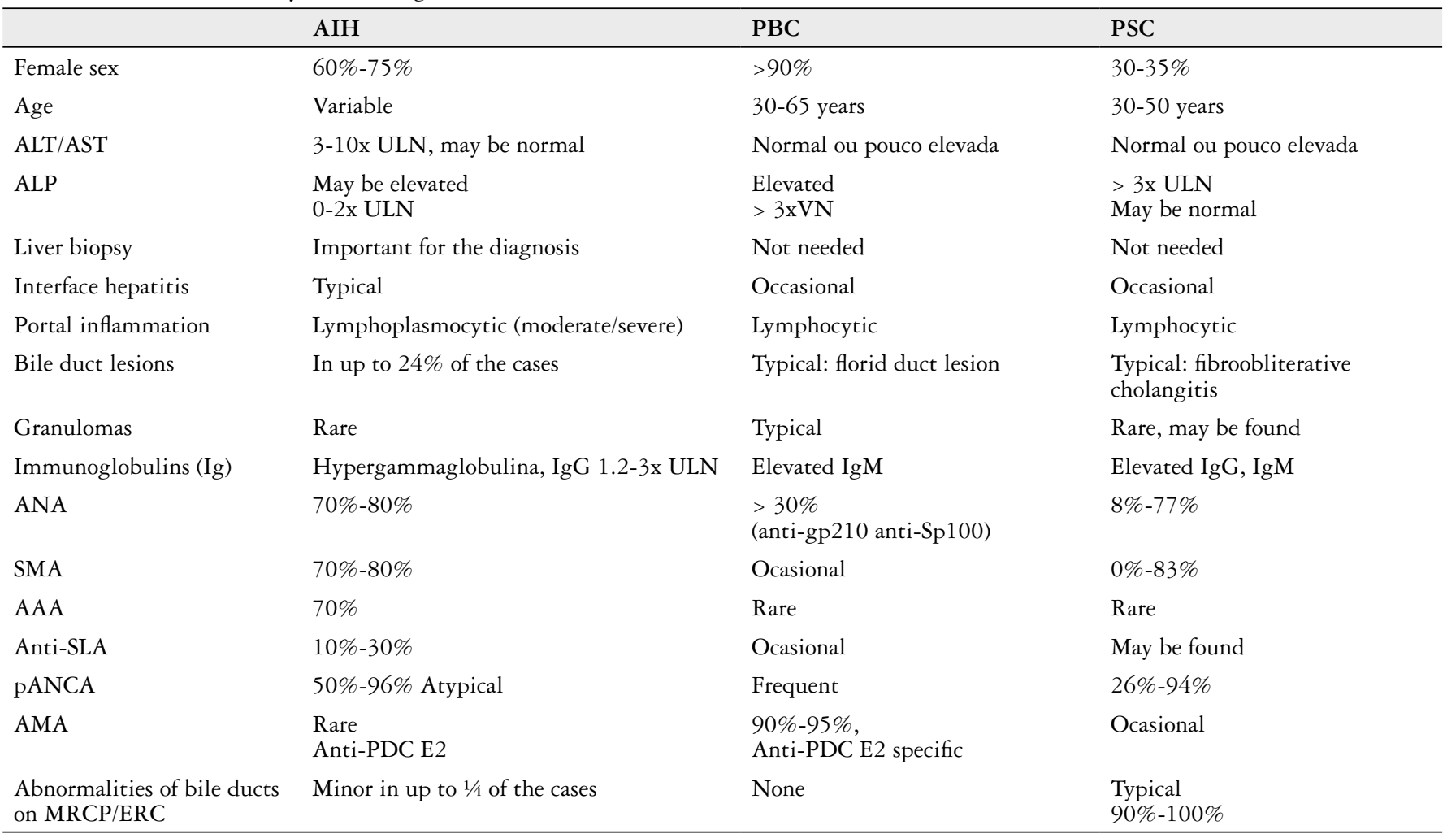

Adapted from ${ }^{(2,7,10)}$; AIH: autoimmune hepatitis; PBC: primary biliary cirrhosis; PSC: primary sclerosing cholangitis; ULN: upper limit of normal; ANA: antinuclear antibody; SMA: anti-smooth muscle antibody; AMA: antimitochondrial antibody; SMA: anti-smooth muscle antibody; AAA: antiactin antibody; ALP: alkaline phosphatase; AST: aspartatoaminotransferase; ALT: alaninoaminotransferase; MRCP: magnetic resonance cholangiopancreatography; ERC: endoscopic retrograde cholangiography 
LT were reported in subjects with AIH-PSC overlap, when compared to their counterparts with $\mathrm{AIH}^{(8,175-178)}$, but its prognosis was shown to be better when compared to pure PSC ${ }^{(176)}$. Regarding AIH-PBC overlap, several studies have shown lower response to ursodeoxicholic acid (UDCA), higher risk of disease progression and decompensation, when compared to classical $\mathrm{PBC}^{(139)}$.

More than three hundred patients with overlap syndromes have been reported in the literature, including case reports and cohorts of patients, due to the concurrent presence of patients with AIH with cholestatic or mixed biochemical profile, IgM elevation, bile duct lesions or granulomas at histology, absence of serological markers or alternatively presence of AMA or anti-M2, absence of response to IS, association with inflammatory bowel disease and cholangiographic findings typical of PSC, as well as PBC subjects with disproportionate elevation of aminotransferases, IgG elevation, presence of high-titer autoantibodies with the exception of AMA, prominent portal and lobular inflammation and response to IS; and large-duct or small-duct PSC patients with disproportionate elevation of aminotransferases, IgG elevation, presence of high-titer autoantibodies, prominent portal and/or lobular inflammation, granulomas and response to $\mathrm{IS}^{(8,175-178)}$.

Several papers have been published with the intention to better characterize AIH-PBC overlap using several diagnostic descriptive criteria and scores, including Paris criteria (Figure 3$)^{(178)}$ and the original, revised and simplified criteria of the International Autoimmune Hepatitis Study Group (IAIHSG) ${ }^{(176)}$. In those reports, AIH-PBC overlap was identified in $1 \%-11 \%, 7 \%, 3 \%-25 \%$ e $6 \%$ of the patients with the initial diagnosis of either AIH or PBC employing, respectively, the Paris and the original, revised and simplified IAHSG criteria. Using the same strategy, other authors have reported a frequency of AIH-PSC overlap in 7,4\%-14\% of the patients with PSC using the revised AIHSG criteria $^{(176)}$. On the other hand, cholangiographic abnormalities, typical of PSC, were reported in $49 \%$ of the children with AIH type 1 and only in $1,7 \%-10 \%$ of the adults with the disease $^{(8,179,180)}$. It should be pointed out that the diagnosis of overlap syndromes of ALD is not straightforward. Proper histological evaluation and cholangiography are essential

\begin{tabular}{|l|}
\hline AIH (two or more parameters) \\
ALT $>5 \times$ ULN \\
SMA ou IgG $>2 \times$ ULN \\
Interface hepatitis (moderate/severe) at liver biopsy \\
PBC (two or more parameters) \\
ALP $>2 \times$ ULN or GGT $>5 \times$ VN \\
AMA \\
Chronic granulomatous cholangitis at liver biopsy
\end{tabular}

FIGURE 3. Paris criteria for the diagnosis of AIH/PBC overlap Adapted from ${ }^{(7)}$; ULN: upper limit of normal; AMA: antimitochondrial antibody; SMA: anti-smooth muscle antibody; ALP: alkaline phosphatase; GGT: gammaglutamyltransferase; ALT: alaninoaminotransferase. to establish their diagnosis as well as the biochemical profile. Due to the lack of standardization, a position paper of the IAIHSG have not recommended the diagnosis of a clear-cut overlap syndrome of AIH-PBC or AIH-PSC, but instead the characterization of one dominant ALD, either AIH, PBC or PSC, with the presence of minor features of another ALD ${ }^{(176)}$. They have also recommended against the use of IAIHSG criteria, developed for the diagnosis of $\mathrm{AIH}$, as a tool for the diagnosis of overlap syndromes. The European Association for the Study of the Liver (EASL), on the other hand, recommended the employment of the Paris criteria for the diagnosis of AIH-PBC overlap, with the consideration of the importance of interface hepatitis in the characterization of the syndrome ${ }^{(68)}$. In this regard, histological and cholangiographic parameters are crucial to characterize either AIH with features of PSC or PSC with features of $\mathrm{AIH}$. On the other hand, disproportionate elevation of aminotransferases, higher than 5 times the upper limit of normal, as well as the presence of interface hepatitis are important to characterize PBC with features of $\mathrm{AIH}$ and vice versa ${ }^{(68)}$.

Immunossupression with corticosteroids with or without azathioprine is the treatment of choice for AIH. The use of UDCA is recommended for PBC in order to reduce disease progression with a positive impact in transplantation-free survival. Its employment in PSC in conventional (13-15 mg/ $\mathrm{kg} /$ day) or moderately higher $(17-23 \mathrm{mg} / \mathrm{kg} /$ day $)$ doses was associated with biochemical response, but adverse outcomes were recently reported with the use of higher doses (28-30 $\mathrm{mg} / \mathrm{kg} /$ day) with higher risk of disease decompensation and death ${ }^{(177,181)}$. There are no well-designed studies with appropriate number of patients to guide evidence-based treatment of AIH-PBC and AIH-PSC overlaps. Some case reports and small retrospective and prospective studies have evaluated the treatment of those disorders with IS, UDCA+IS and UDCA. Most of them have reported some degree of biochemical response with IS with or without UDCA ${ }^{(176,178)}$. It should be emphasized that diagnostic criteria for overlapping syndromes of ALD and treatment end-points were not standardized in most of those reports. Studies from Germany, Japan and Italy ${ }^{(178,182,183)}$, including 58 patients with AIH-PBC overlap treated with UDCA+IS have disclosed biochemical response in most of those treated subjects. Other French study. Other French study, evaluating treatment response of 17 patients with AIH-PBC overlap AIH/PBC with either UDCA $(n=11)$ or UDCA+IS $(n=6)$ followed for 7,3 years, have observed more frequent biochemical response and less fibrosis progression in the group of patients with combined UDCA+IS ${ }^{(184)}$. Distinct results were reported in another study (15) which disclosed no difference in the treatment of those patients either with UDCA or with UDCA+IS ${ }^{(185)}$. A recent meta-analysis, however, showed more biochemical and histological improvement in subjects treated with UDCA+IS when comared to those treated with UDCA alone ${ }^{(186)}$. The IAIHSG recommends treatment individualization of $\mathrm{PBC}$ subjects with features of AIH and vice versa ${ }^{(176)}$. The use of UDCA can be associated with IS (steroids with or without 
azathioprine) for treatment of those subjects or alternatively can be used first with the subsequent addition of IS in the presence of non-response ${ }^{(139,176)}$. Case reports have also disclosed biochemical response to cyclosporine or mycophenolate in substitution to azathioprine in refractory cases ${ }^{(139,176)}$.

Treatment of PSC with features of IS and UDCA+IS was also associated with some degree of biochemical response in several case reports and small cohorts of patients ${ }^{(176,181)}$, with higher response rates observed in children ${ }^{(8)}$. Small studies from the Netherlands $(n=9)$ and Italy $(n=7)$ have reported biochemical response in most of the patients treated with IS or UDCA+IS ${ }^{(187,188)}$. Subsequent studies from United Kingdom and Sweden have described biochemical response, respectively in 14 out of 16 and 16 out of 24 treated patients with IS ${ }^{(178,189)}$. Other German study ${ }^{(190)}$ also disclosed biochemical response with IS with or without UDCA in most of the patients, but, even in the presence of biochemical response, disease progression was observed $3 / 4$ of the patients after a median follow-up of 12 years. In children, biochemical response rates were shown to be higher, when compared to adults, with a decrease in liver enzymes reported in 23 out of 27 treated patients under UDCA+IS ${ }^{(8)}$.

The IAIHSG recommends treatment individualization of PSC subjects with features of AIH and vice versa ${ }^{(176)}$. Use of IS (steroids with or without azathioprine) was recommended for treatment of those subjects. Combination of UDCA with IS was further recommended by EASL ${ }^{(68)}$, but not by American Association for the Study of the liver, who recommends against the use of UDCA for PSC alone or with features of $\mathrm{AIH}^{(75,176)}$.

\section{Recommendations}

- Autoimmune diseases of the liver should be categorized according to their predominant features as $\mathrm{AIH}$, PBC, PSC and small-duct PSC. Overlap syndromes should not be viewed as distinct diagnostic entities. In the presence of relevant overlapping clinical, laboratory, histological and cholangiographic features of more than one ALD in the same patient, the diagnosis should be based on the preponderant disease: AIH, PBC, PSC or small-duct PSC with the addition of the presence of features of another ALD (Class IIb).

- IAIHSG diagnostic criteria should not be used to diagnose overlap of AIH with either PBC or PSC (Class IIa).

- Patients with AIH with features of either PBC or PSC and vice versa should be referred to centers with expertise in diagnosis and treatment of ALD (Class IIb)

- Paris criteria can be useful to characterize AIH-PSC overlap, but are not entirely validated to be used in clinical practice (Class IIb).

- Patients with PSC and PBC with features of AIH should be considered for treatment with immunossupression (Class IIb)

- Combined treatment of UDCA and imunossupression can be considered for patients with AIH with features of PBC (Class IIb).
- Use of combined treatment of UDCA in conventional doses and immunossupression for patients with AIH with features of PSC may be benefitial, but data is even more scarce regarding treatment end-points (Class IIb).

\section{PART V: COMPLICATIONS OF CHOLESTASIS}

\section{Pruritus}

Pruritus is a sensory phenomenon related to a broad range of systemic disorders including endocrine, metabolic, hepatobiliary, lymphoproliferative and infectious diseases. In hepatobiliary diseases, pruritus is particularly associated with cholestasis due to an impairment of bile formation and/or bile flow. It may be caused by hepatocellular and/or cholangiocellular cholestasis as well as by obstruction of the intra or extrahepatic bile ducts.

The prevalence of pruritus was shown to vary considerably in different liver diseases. It is considered as the defining feature of intrahepatic cholestasis of pregnancy (ICP) and is observed in $70 \%-80 \%$ of the patients with PBC and PSC, as well as in $16 \%-45 \%$ of those subjects with obstructive cholestasis due to biliary stones or tumors ${ }^{(191)}$. It may be mild and tolerable but it may also dramatically reduce the quality of life of affected patients with sleep deprivation, fatigue, depressive mood and suicidal ideations. It has a circadian cycle with the highest intensity observed in the evening hours or nighttime. Itching can be generalized but often involves preferentially the limbs, palms and soles. The skin is devoid of primary lesions but excoriations and prurigo nodularis can be found. The pain induced by vigorous scratching is associated with relief of pruritus. In female patients, it exacerbates in the premenstrual period and in late pregnancy ${ }^{(192-194)}$.

The precise mechanism of cholestatic pruritus remains unclear. Recent studies showed that itching and pain are transmitted by different afferent fibers. Pruritogens may bind to their receptors on itch nerve ending in the skin. Histamine receptors, PAR2, IL-31 receptor, TRP receptor family, Mrg receptor family and lysophosphatidic acid (LPA) receptors are involved. Recent findings indicate that LPA, a potent neuronal activator, and autotaxin (ATX), the enzyme that forms LPA, may play a key element in the pathogenesis of cholestatic pruritus. Bile salts, histamine, $\mu$-opioids, and serotonin have been controversially involved in the pathogenesis of cholestatic pruritus. However, for those substances neither a correlation between serum and/or tissue concentration and itching severity nor a causative link have ever been established. Serum activity of ATX correlates with itching intensity and response to treatment in patients with cholestatic pruritus but not with other forms of itching ${ }^{(191-193)}$.

Treatment options for pruritus in cholestasis remain limited to a few evidence-based therapies ${ }^{(68,193)}$. Therapeutic interventions should primarily focus on an adequate therapy of the underlying hepatobiliary disease, such as: 1 ) to remove of the pruritogens from the enterohepatic cycle by nonabsorbable anion exchange resins such as cholestyramine 4-16 g per day. Resins should be administered away from other drugs; 2) to change the metabolism of the presumed 
pruritogens in the liver and /or the gut by biotransformation using enzyme inducers such as rifampicin 150-600 mg per day, regarded as second-line treatment; 3 ) to modify central itch and/or pain signaling by influencing the endogenous opiodergic and serotoninergic system via opioid antagonist and selective serotonin reuptake inhibitors. Naltrexone, an oral opiate antagonist, at dose $12,5-50 \mathrm{mg}$ daily, should be considered as third line treatment, after failure or intolerance to cholestyramine and rifampicin. Sertraline $(100 \mathrm{mg} /$ day $)$ may also be considered for patients refractory to aforementioned drugs; 4) to remove the potential pruritogens from the circulation by invasive methods such as nasobiliary and transcutaneous drainage, plasmapheresis and extracorporeal albumin dialysis. Invasive approaches should be considered for unresponsive patients. There is no evidence to suggest that UDCA lessens cholestatic itching, with exception of pruritus associated with ICP. The use of antihistamines is not recommended due to lack of efficacy ${ }^{(68)}$.

Itching quantification using a visual analogue scale (VAS) can be helpful in the assessment of response to drug intervention with 0 to 3,4 to 8 and 9 to 10 scales considered, respectively as mild, moderate and severe pruritus ${ }^{(195)}$. In addition, the 5 - $D$ pruritus scale is a valuable tool to quantify chronic prutritus, exhibiting a high correlation with the VAS ${ }^{(195)}$.

Cholestyramine, rifampicin, naltrexone and sertraline are recommended by European ${ }^{(68)}$ and American Guidelines ${ }^{(121)}$ and should be prescribed in a stepwise manner in order to control the cholestatic pruritus. Patients unresponsive to the evidence-based guideline approved drugs should be considered as refractory to treatment. They can undergo experimental therapies such as oral ondansetron (4-24 mg/ day), oral phenobarbital (2-5 mg/kg/day), propofol (10-15 $\mathrm{mg}$ iv bolus, followed by $1 \mathrm{mg} / \mathrm{kg} /$ hour $)$, lidocaine $(100 \mathrm{mg} /$ day), phototherapy UVB, extracorporeal albumin dialysis, plasmapheresis, nasobiliary drainage and biliary diversion. LT can be regarded as the last step, when all available interventions have been proved to be ineffective ${ }^{(121,128,192,196,198)}$.

LT raises issues of organ allocation priority. In refractory cases MELD exception points should be evaluated.

\section{Recommendations}

- Pruritus is frequently observed in cholestatic liver diseases and tend to decrease in frequency and intensity with disease progression to cirrhosis. (Class I)

- Treatment of pruritus should be stepwise until resolution or improvement of symptoms using 1rst line drugs such as cholestyramine (4-16 g/daily), $2^{\text {nd }}$ line drugs such as rifampicin $(150-600 \mathrm{mg} /$ daily $), 3^{\text {rd }}$ line drugs such as naltrexone (12,5-50 mg/daily) and fourth line drugs such as sertraline (50-100 mg/daily) (Class I- IIa)

- The presence of refractory pruritus should be considered in the presence of failure to control itching under maximal doses of cholestyramine, rifampicin, naltrexone and sertraline (Class I)

- Use of antihistamines and UDCA could not be recommended for treatment of pruritus, with the exception of UDCA in ICP (Class I )
- LT should be considered in cases of refractory pruritus in the absence of effective treatment measures and interventions. (Class IIb)

\section{Fatigue and Hypercholesterolemia}

Fatigue is a common symptom in clinical practice, particularly in subjects with PBC. It is estimated that 40\%-80\% of the patients with $\mathrm{PBC}$ would have fatigue. Half of them will complain of fatigue as their leading symptom of liver disease ${ }^{(128,197,198)}$. Some authors consider PBC as a systemic disorder, inasmuch as the physiopathology of fatigue was shown to involve degenerative changes in the central nervous system, in domains critical for sleep and autonomic function, and mitochondrial dysfunction leading to increased anaerobic muscle activity. The intensity of fatigue shows no correlation with liver disease severity. The evaluation and treatment of fatigue in cholestasis are complex. It is necessary to rule out other causes such as depression, anemia, hypothyroidism, adrenal failure as well as use of antidepressants, antihistamines and antihypertensives ${ }^{(68,199)}$. Around $40 \%$ of the patients with PBC and PSC have depression, not well evaluated by psychiatric questionnaires, but fatigue usually does not improve with the treatment for depression ${ }^{(199,200)}$. Somnolence can mimic fatigue and is usually associated with hypothyroidism, seen in $20 \%$ of PBC subjects ${ }^{(201)}$. Treatment of fatigue in cholestasis is challenging, as the use of several agents such as UDCA, antidepressants and ondansentron was not shown to influence fatigue scores. Some studies have observed improvement of those scores with the use of modafinil, usually employed for narcolepsy treatment. Use of modafinil (100-200 mg/daily) in $42 \mathrm{PBC}$ patients with severe fatigue was associated in one study with improvement of symptoms in $74 \%$ of treated patients in the first three days of therapy ${ }^{(205)}$. Randomized studies are needed before recommendation of modafinil for fatigue in cholestasis. Measures including frequent bed rest periods during the day, avoidance of sleep deprivation with abstinence of caffeine in the afternoon and psychological support seems to be important in the management of fatigue. LT can be considered for incapacitating fatigue, but is usually not associated with quick resolution of symptoms. In this regard, one recent study including 49 patients with $\mathrm{PBC}$ with fatigue, submitted to LT, reported improvement of fatigue scores in most of the patients, but half of them had persistent moderate to severe fatigue for more than two years after surgery ${ }^{(203)}$.

Hyperlipidemia with abnormal total cholesterol (TC) levels and elevated LDL and HDL-cholesterol is reported in most of the patients with cholestatic liver disorders, particularly PBC. Reduction of TC and LDL-cholesterol is seen with disease progression and treatment with $\mathrm{UDCA}^{(68,204)}$. Hyperlipidemia in cholestasis is due to accumulation of lipoprotein X. The main clinical features of hyperlipidemina in cholestatic liver diseases are the occurence of xanthelasmas and xantomas. Different from other dyslipidemias, the hyperlipidemia of cholestasis is not associated with accelerated atherosclerosis and higher risk of cardiovascular events in the absence of other known risk factors for coronary and cerebrovascular diseases ${ }^{(121,204)}$. 
Hepatotoxicity has been reported with statins, but their use is reported to be safe in patients with liver diseases with or without cholestasis. In this regard, pravastatin was shown to be safe in patients with hepatitis $\mathrm{C}$ and nonalcoholic steatohepatitis ${ }^{(205)}$. The use of simvastatin in subjects with PBC was also not associated with hepatotoxicity and was useful to reduce cholesterol and LDL-cholesterol levels ${ }^{(206)}$. The main question regarding the use of statins in patients with $\mathrm{PBC}$ or PSC is not related to potential adverse events but to its real indication for lowering TC and LDL-cholesterol, due to the lower risk of cardiovascular events reported in those subjects $^{(68,121)}$. On the other hand, it is clear that subjects with cholestasis additional risk factors for atherosclerosis may benefit from the use of lipid lowering drugs such as statins, fibrates and cholestyramine.

\section{Recommendations}

- Fatigue is frequently seen in cholestatic liver diseases, particularly PBC (Class I)

- Exclusion of depression, anemia, hypothyroidism and fatigue-inducing drugs should be carried out (Class IIa)

- There is no approved treatment for fatigue and LT can be considered for severe and incapacitating fatigue, particularly in PBC (Class IIb)

- Frequent bed rest, avoidance of sleep deprivation and psychological support are important in the management of fatigue (Class IIa)

- Hyperlipidemia with high total cholesterol, LDL and HDL-cholesterol is frequently found in subjects with cholestasis, particularly in PBC (Class I)

- There is no data to support higher risk of atherosclerosis and cardiovascular events in subjects with cholestasis (Class IIb)

- Statins, when required, are considered safe and effective for treatment of hyperlipidemia in cholestatic liver diseases. (Class IIb)

\section{Osteoporosis and osteopenia}

The prevalence of osteoporosis and bone fractures in PBC is higher when compared to other chronic liver diseases. In this respect, osteoporosis and spontaneous fractures were reported to occur, respectively, in 20\%-37\% and 13\%-22\% of the subjects with $\mathrm{PBC}$ and in $6 \%$ and $15 \%$ of the patients with PSC(207).

The risk of osteopenia, osteoporosis and fractures increases even more after LT. Most patients suffer from accelerated bone loss three to six months after surgery, with restoration of preoperative bone mass after two years of $\mathrm{LT}^{(208)}$. A slight reduction of bone mineral density (BMD), that occur after transplantation, is enough to increase the risk of fractures, which are observed in 25\%-35\% in the first year after the procedure ${ }^{(209)}$. Bone loss after LT is due to high doses of corticosteroids and other immunossupressors such as tacrolimus and cyclosporin.

Bone densitometry is the gold-standard test for the diagnosis of osteopenia and osteoporosis. It should be performed in subjects with history of spontaneous fractures, in the awaiting list or after for LT and use of doses higher than $5 \mathrm{mg} /$ daily of prednisone for AIH. Bone densitometry is also highly indicated for patients with PBC and PSC and subjects with liver disease with or without cholestasis that have other risk factors for bone loss, such smoking, alcohol abuse, hypogonadism, amenorrhea or lower body mass index (BMI ${ }^{(68,210)}$. The test should be repeated at two to three years in patients without bone loss at the first examination. Annual reevaluation is indicated in subjects with advanced cirrhosis, after LT as well as in those treated with high doses of corticosteroids ${ }^{(68,210)}$.

Each patient with osteopenia or osteoporosis should be advised to abstain from alcohol and smoking and to avoid high amounts of coffee drinking. Regular exercise should also be emphasized as well as a balanced diet rich in calcium and vitamin D. Whenever possible, corticosteroids should be tapered to the lower dose or withdrawed. Patients with cirrhosis are at an increased risk for bone loss and should receive supplementation of calcium (1000-1500 mg/daily) and vitamin D (400-800 IU/daily), irrespective of BMD results. In the presence of vitamin D deficiency, administration of large doses of vitamin D (50.000 IU a week for eight weeks) are required to maintain vitamin D levels to at least 25-30 $\mathrm{ng} / \mathrm{ml}$. Special attention should be taken in subjects under cholestyramine due to its effect in vitamin D.

Treatment of bone loss with bifosfonates is indicated in patients with osteoporosis with evidence of spontaneous fractures and with prolonged use of corticosteroids. Patients with chronic cholestasis and osteopenia with a $\mathrm{T}$ score $<1,5$ should also be treated with bifosfonates. They are also indicated for prevention of accelerated bone loss after LT and should be employed before or just after surgery ${ }^{(209,210)}$. Some studies evaluating the effect of bifosfonates in PBC patients have reported increased bone mass with the use of theses agents, with no decrease in the frequency of spontaneous fractures. Alendronate and ibandronate have been tested in PBC with similar results regarding safety and BMD, but a recent study ${ }^{(211)}$ reported better adherence to treatment with once-a-month schedule of $150 \mathrm{mg}$ of ibandronate.

The use of intravenous pamidronate after LT was associated with conflicting results, but oral alendronate in this setting was beneficial in regard to bone loss prevention, without influence in the rate of fractures ${ }^{(210)}$. Use of intravenous zoledronic acid ${ }^{(212,213)}$ in doses of $4 \mathrm{mg}$ every three months was shown to be efficient in the prevention of bone loss and fractures after LT. Recently, oral or intravenous ibandronate $^{(214,215)}$, was also shown to improve BMD and to reduce the incidence of fractures after LT.

\section{Recommendations}

- Bone densitometry is the gold-standard test for the diagnosis of bone loss and should be performed in subjects with history of spontaneous fractures; chronic use of corticosteroids; diagnosis of PBC and PSC; under evaluation or after LT; advanced cirrhosis or cholestasis, irrespective of etiology, with additional risk factors for bone loss (Class I). 
Bittencourt PL, Cançado ELR, Couto CA, Levy C, Porta G, Silva AEB, Terrabuio DRB, Members of the Pannel of the 1st Consensus of the Brazilian Society of Hepatology on the Diagnosis and Management of Autoimmune Diseases of the Liver. Brazilian Society of Hepatology recommendations for the diagnosis and management of autoimmune diseases of the liver

- Bone densitometry should be repeated every two to three years in subjects without bone loss at the first test. Annual reevaluations are necessary in subjects with advanced cirrhosis, after LT or with the use of high doses of corticosteroids (Class I).

- Management of bone loss should involve lifestyle changes, including avoidance of smoking, alcohol and excessive coffee drinking. Physical activity and a wellbalanced diet rich in calcium and vitamin $\mathrm{D}$ should be recommended. Supplementation of calcium (1000$1500 \mathrm{mg} /$ daily) and vitamin D (400-800 IU/daily), should be considered, independently of BMD results in patients at increased risk for bone loss (Class IIa).

- Use of bisfosfonates should be considered in the presence of osteoporosis ( $\mathrm{T}$ score $<-2,5$ ), spontaneous fractures, before and after LT, prolonged use of corticosteroids and chronic cholestasis with $\mathrm{T}$ score $<-1,5$. Alendronate and ibandronate can be employed without distinction, but treatment adherence is more with ibandronato (Class IIa).

\section{PART VI: SPECIAL TOPICS}

\section{Recurrent Cholangitis}

Patients with structural changes in the biliary tree due to PSC, Caroli syndrome or ischemic cholangitis are at high risk for recurrent bacterial chlangitis ${ }^{(68)}$. Biliary stasis due to obstruction of bile flow are associtated with bacterial colonization, which may evolve to cholangitis. Recurrent cholangitis is characterized by relapsing bouts of fever with or without chills, jaundice and abdominal pain ${ }^{(216)}$. Those symptoms, nevertheless, may be absent in some patients, who may exhibit inespecific complaints or be entirely assymptomatic with only abnormal liver enzymes ${ }^{(70)}$. Sometimes, culture-proven bacteremia of unknown site could be found as the sole manifestation of cholangitis in those patients ${ }^{(68)}$. Episodes of cholangitis may occur spontaneously or after invasive diagnostic or therapeutic procedures in the biliary tree, such as endoscopic retrograde (ERC) or percutaneous cholangiography. Despite the use of prophylactic antibiotics, recurrent cholangitis in usual in this setting, evolving in some patients to sepsis and septic shock. In those patients with cholangitis, LT is usually not contraindicated, inasmuch as infection is adequately controlled with antibiotics ${ }^{(217)}$.

In 2006, a consensus conference sponsored by the Organ Procurement and Transplantation Network (OPTN) ${ }^{(218)}$ defined the requirement of MELD/PELD score exception points for subjects with recurrent cholangitis under a higher risk of mortality, not adequately assessed by the MELD scoring system. Recommendations for granting extra-MELD points for reccurent cholangitis included the occurrence of two or more episodes of culture-proven cholangitis in a 6-month period or the development of septic complications such as liver abscess, bacterial meningitis, endocarditis and osteomyelitis as well as fungemia. Subjects with procedurerelated bacteremia due to ERC or percutaneous cholangiography or with biliary stents were not considered candidates for MELD-exception points. In order to apply for additional points, candidates for LT with reccurent cholangitis should have structural changes in the biliary tree, not amenable to treatment and previous use of antibiotics for the treatment of all infection episodes.

Subsequenly in 2008, the French Association for the Study of the Liver defined their own criteria for prioritization of subjects with recurrent cholangitis ${ }^{219}$ based on the following parameters, which were better associated with adverse outcomes:

a) Biliary disease refractory to endoscopic or percutaneous treatment with more than two episodes of bacterial cholangitis in the last six months or at least one serious infection episode, including liver abscess or extra-hepatic sepsis. Access for LT in this setting was suggested to to be in less than 6 months.

b) Cholangitis with septic shock or due to infection with multiresistent bacteria as well cholangitis due to intractable biliary complications after LT. Access for LT in this setting was suggested to to be in less than 3 months.

Cholangitis and recurrent cholangitis may be the initial symptoms of PSC in $6 \%$ and $10 \%$ of the cases, respectively ${ }^{(6)}$. Although there is no evidence of increased mortality or drop out rates of PSC patients in the waiting list for LT due to complications of cholangitis, the adverse impact of recurrent cholangitis in the morbimortality of those patients is a real concern ${ }^{(221,218,222)}$. In this respect, several Guidelines suggest prioritization of those patients for LT under special circunstances highlighted ${ }^{(218,219)}$.

Cholangitis and sepsis can occur in 3\% to $23 \%$ of the cases after $\mathrm{ERC}^{(216,223)}$, particularly in those subjects with risk factors such as biliary obstruction and inadequate drainage of the biliary tree after ERC (223). Use of prophylactic antibiotics before or durimg ERC is controversial, but several studies have reported a decrease in infectious complications in patients under antibiotic coverage ${ }^{(224)}$, particularly in the presence of biliary obstruction ${ }^{(225,226)}$. The American and British Societies of Gastrointestinal Endoscopy recommend antibiotic prophylaxis for patients submitted to ERC in the presence of biliary obstruction ${ }^{(227,228)}$.

In subjects with recurrent cholangitis, employment of long-term antibiotics for prevention of subsequent episodes of cholangitis has been suggested ${ }^{(75)}$, either one drug used continuously or in rotation with other antibiotics, including oral amoxicillin-clavulanate, ciprofloxacin and cephalexin. However, there is no evidence-based data to support such strategy ${ }^{(75,218)}$.

Patients with PSC with dominant strictures as well as patients with other biliary tract diseases with recurrent cholangitis should be evaluated for elegibility of endoscopic treatment. The endoscopic management of PSC subjects with dominant strictures has been highlight elsewere in this manuscript. Other treatment modalities for biliary drainage such as Roux-en-Y hepaticojejunostomy is controversial in this setting due to higher surgical morbimortality associated with the presence of CLD as well the eventual subsequent requirement of LT. 


\section{Recommendations}

- Patients with recurrent cholangitis due to biliary tract disease refractory or not amenable to medical, endoscopic or surgical treatments should receive prioritization for LT (Class I).

- MELD-exception points should be given to those patients with recurrent cholangitis in the presence of: a) two or more episodes of cholangitis in at least 6 months; b) one episode of recurrent cholangitis with extrahepatic sepsis, severe sepsis or septic shock (not associated with biliary tract procedures) or c) due to infection with multiresistant bacteria (Class IIa).

- Antibiotic prophylaxis shoud be given to those patients with PSC or with any other disease associated with biliary obstruction submitted to ERC in order to prevent cholangitis, particularly in the presence of inadequate biliary drainage (Class IIa).

\section{Screening and management of cholangiocarcinoma}

Patients with PSC have a 100-fold higher risk of biliary tumors, when compared to the general population, with a reported prevalence of 5\%-15\%(238-243). The most common type of cancer is CC. Half of the cases of CC are reported in the first year after the diagnosis of PSC ${ }^{(239,241)}$. Patients with concurrent IBD are at increased risk for CC. Other reported risk factors are increasing age, alcohol, smoking, longstanding IBD and present or past diagnosis of colorectal cancer. CC can also be encountered in cystic biliary diseases, including Caroli disease ${ }^{(242)}$.

Aggravation of jaundice as well as clinical and biochemical deterioration are common findings in patients with $\mathrm{CC}$, but they are also usually found in subjects with progressive PSC without cancer ${ }^{(238-242)}$.

Gallbladder cancer can be found in subjects with PSC, with a lifetime prevalence of $2 \%{ }^{(238,241)}$. The risk of pancreatic cancer is also 14-times increased in patients with PSC, when compared to the general population.

There is no current evidence-based strategy recommended for the screening of $\mathrm{CC}$ and gallbladder cancer in patients with PSC.

In regard to $\mathrm{CC}$, screening strategies employing imaging techniques, preferentially with MRI, but also ultrasound and computed tomography, in association with tumor biomarkers have been suggested strategies ${ }^{(241,244)}$, but their accuracy in detecting CC was reported to be low ${ }^{(24,245)}$.

The most commonly employing biomarker for $\mathrm{CC}$ in PSC is CA19-9. Higher values of CA19-9 were reported for patients with PSC and CC, when compared to those counterparts without cancer. However, there is no welldefined cut-off level, which can be used to differentiate PSC patients with and without CC. Values higher than $20 \mathrm{U} / \mathrm{ml}$, when employed in conjunction to MRCP, can increase their specificity to levels near $100 \%$, but with lower sensitivity (less than $40 \%$ ). In addition, CA19-9 levels equal or higher than $129 \mathrm{U} / \mathrm{ml}$ have also been shown to increase specificity, but also with a significant decrease in sensitivity ${ }^{(244,245)}$. Higher levels of CA19-9 were also associated with advanced tumors, usually with adverse outcomes ${ }^{(241,245,246)}$.
Endoscopic retrograde colangiography with brushing citology is a method currently used for diagnostic confirmation of CC in PSC subjects with dominant strictures and/or with abnormal levels of CA19-9, but not for tumor screening due to its association with pottentialy serious adverse events $^{(241,244,247)}$. The adittion of fluorescent in situ hybridization (FISH) of cytologic samples, disclosing polysomy in at least five biliary cless obtained by endoscopic brushing, was shown to enhance diagnostic accuracy for CC in PSC ${ }^{(68,75)}$.

Both AASLD ${ }^{(75)}$ and EASL ${ }^{(68)}$ guidelines, do not propose a well-defined strategy for the screening of CC in PSC, recommending ERC with brushing citology for only for those subjects with suspected cancer based on clinical and laboratory grounds. Ultrasound every year is nevertheless indicate for screening of gallbladder cancer. In the presence of any solid lesion in the gallbladder cholecystectomy should be performed $^{(247)}$.

\section{Recommendations:}

- Patients with PSC are at increased risk for CC and gallbladder cancer (Class I)

- In the absence of evidence-based data, ultrasound should be performed at least yearly for screening of $\mathrm{CC}$ in association with measurement of CA19-9 levels (Class IIb). MRCP should be preferred in those patients with suspected $\mathrm{CC}$ based on clinical and laboratory findings. (Class IIb). ERC with brushing cytology is recommended to stablish the diagnosis of biliary tract cancer.

- Screening of gallbladder cancer should be performed with yearly ultrasound in subjects with PSC. In the presence of polyps of any size or any other lesions, cholecystectomy should be performed (Class IIa)

- Screening for hepatocelular carcinoma should be performed every six months in subjects with cirrhosis due to PSC (Class IIa).

\section{Liver transplantation (LT) for AIH, PBC and PSC}

Autoimmune hepattis, $\mathrm{PBC}$ and PSC are autoimmune or immune-mediated diseases of the liver that often progresss to cirrhosis and liver failure. Consideration for LT is warranted in those subjects with end-stage liver diasease, hepatocellular carcinoma or with incapacitating symptoms of recurrent cholangitis, fatigue and prutitus. Primary sclerosing cholangitis and AIH are responsible, respectively, for $4 \%$ and $4 \%-6 \%$ of the patients with liver diseases requiring LT worldwide. In Scandinavia, where the disease is very prevalent, $16 \%$ of the LT are due to PSC.

The prevalence of $\mathrm{PBC}$ is increasing in recent years, but the percentage of LT performed for PBC have decreased from $8 \%$ to $4 \%$ in the last 20 to 30 years, possibly due increased disease awareness with its diagnosis at early stages as well as increased disease survival with UDCA ${ }^{(248)}$.

The indication of LT for ALD is usually clear with the advent of complications associated with portal hypertension or liver failure, such as ascitis, hepatic encephalopathy, variceal hemorrhage and spontaneous bacterial peritonitis as 
well as with the development of hepatocarcinoma. Survival benefit of LT is usually seen in subjects with MELD scores equal or greater than 15 or with CPS scores B and C.

MELD maior ou igual a 15 ou classificação de ChildTurcotte-Pugh modificada B ou C. The incidence of HCC in AIH and PSC was reported to be around 2\% per year. In $\mathrm{PBC}$, on the other hand, the prevelance of HCC was estimated as $4 \%$ to $12 \%$ in 10 years. Indication and prioritization for LT in this setting is not different from those related to HCC in other chronic liver diseases ${ }^{(68,248-249)}$.

Refractory pruritus in PBC and PSC, as well as recurrent refractory cholangitis in PSC are also recognized indications for LT that require evaluation for extra-MELD points for prioritization ${ }^{(68,75,248,249)}$, as outlined elsewhere in this manuscript. Fatigue, on the other hand, is a serious complication of PSC and PBC, which is associated with decreased quality of life. It is a subjective symptom, very difficult to measure and may persist after LT. It is usually not considered with extra-MELD points for prioritization ${ }^{(68,75,248)}$.

The EASL guidelines recommend referral of PBC patients for LT when total bilirubin is equal or higher than 6 $\mathrm{mg} / \mathrm{dl}$, Mayo risk score (MRS) is equal or higher than 7,8 and MELD is higher than $12^{(68)}$. Likewise, consideration for LT was suggested in the presence of ALD, evidence for biliary epithelia dysplasia and recurrent cholangitis ${ }^{(68)}$. The AASLD guidelines, on the other hand, recommend LT for PSC subjects in the presence of end-stage liver disease with complications due to liver filure and portal hypertension, as well as intractable pruritus and recurrent cholangitis. Selected patients with CC can also be considered for LT associated with neo-adjuvant radio-chemotherapy ${ }^{(75)}$.

Organ allocation in most transplantation centers worldwide is based on the MELD score. Even though, the MELD score was initially not considered to be a reliable prognosticator of cholestatic liver diseases ${ }^{(253-256)}$. Other studies, hovewer, have demonstrated that MELD score were accurate to predict mortality of PBC patients on the waiting list for LT. Disease specific criteria, particularly the MRS, were shown to better predict the onset of complications, when compared to MELD and Child Pugh scores, but tend to overestimate mortality risk in PBC patients. These findings were also disclosed in another study, which showed better accuracy of the MRS, when compared to the European and Yale scores, to predict morbimortality of subjects with PBC, but all of them had exhibited low positive predictive values for adverse outcomes, limiting their applicability in clinical practice ${ }^{(257)}$. In respect to PSC, the use of disease-specific prognostic scores are also not recommended ${ }^{(68,75,248)}$.

LT may be warranted in those AIH patients with decompensation of liver disease due to flares of AIH due to poor adherence or spontaneous disease reactivation. In this cases, drug adjustments should be initially employed with concern due to the higher risk for development of infection. In the absence of improvement, LT should be considered.

It is important to point out that $9 \%-20 \%$ of the subjects with AIH may have FHF at onset with requirement for $\mathbf{L T}^{(9)}$. The diagnosis of those patients is challenging, since most of them do not present hypergammaglobulinemia and circulating autoantibodies at presentation and the AIHSG and the simplified criteria are unreliable for the diagnosis of fulminant AIH.

It is noteworthy that up to $20 \%$ of the cases of FHF are of indeterminate etiology. Half of them may have a probable diagnosis of $\mathrm{AIH}^{(258,259)}$. In those patients, prompt institution of therapy can be either lifesaving or avoid LT. There is no consensus regarding the best treatment strategy for those subjects with fulminant AIH. Most centers use intravenous prednisolone $20-100 \mathrm{mg} / \mathrm{day}$ in adults or $1 \mathrm{mg} / \mathrm{kg} /$ day in children. Others prefer oral prednisone 20-30 mg/day (258-260). One-third of the subjects respond to corticosteroids, particularly those with MELD scores equal or less than 28 at admission, absence of massive necrosis on liver biopsy and no improvement or stabilization of RNI and total bilirubin in the first 4 days after treatament. Absence of response with 7 days of therapy usually defines treatment failure, withdrawal of corticosteroids and LT. Mortality of fulminant AIH may as higher as $30 \%$, even in referral centers ${ }^{(258-260)}$.

LT is an excellent treatment option for ALD, with reported 1 -year survival rates above $90 \%$, and 5 to 10 years survival rates of $80 \%-85 \%, 80 \%$ and $80 \%-90 \%$, respectively, for PBC, PSC and $\mathrm{AIH}^{(68,248)}$. Disease recurrence after LT, on the other hand, is observed, respectively in $30 \%-35 \%, 30 \%$ and $12 \%-46 \%$ of the cases of PBC, PSC and $\mathrm{AIH}^{(248,260,261)}$. Risk factors for PSC recurrence after LT are not very well-stablished, while immunossupression with cyclosporine appears to be protective against PBC recurrence after surgery ${ }^{(248)}$. In regard to AIH, the intensity of necroinflammatory activity and higher levels of $\mathrm{IgG}$ are associated with higher risk for recurrence postLT. In adittion, patients with AIH-1, when compared to their counterparts with type 2 disease, have more disease recurrence after $\mathrm{LT}^{(248,260)}$. Life-long corticosteroid therapy have been proposed in order to reduce the risk of AIH recurrence, but the need for corticosteroid maintenance treatment in this setting remains controversial. However, there is general agreement that patients with AIH need more intensive immunossupression after LT with dual or even triple drug therapy to decrease the risk of disease recurrence and graft dysfunction.

\section{Recommendations}

- Patients with AIH, PSC and PBC, as well as with other should be referred for LT in the presence of complications of portal hypertension and liver failure assessed by the MELD score (Class I)

- Intractable pruritus and refractory recurrent cholangitis should be considered for prioritization with extraMELD points in subjects with PBC and PSC (Class I)

- LT has no role in the management of AIH refractory to treatment in the absence of complications of liver failure and portal hypertension. (Class IIa).

- LT may be warranted in those patients with decompensation of liver disease due to flares of AIH due to poor adherence or spontaneous disease reactivation. In this 
cases, drug adjustments should be initially employed with concern due to the higher risk for development of infection. In the absence of improvement, LT should be considered (Class IIb)

- Use of prognostic scores for indication of LT for PSC and PBC still deserve better validation. Until now, MELD remains the best score for indication od LT and organ alocation (Class IIa)

- When LT is considered for AIH, withdrawal of imunossupressive therapy is warranted when LT appears to be iminente (Class IIb)

- Maintenance of UDCA in subjects with PSC and PBC in the awaiting list for LT is controversial, since its impact in the survival of those patients with endstage liver disease is probably negligeble (Class IIb)

- In subjects with acute liver failure, after exclusion of other causes of liver disease, even in the absence of autoantibodies, instituiton of immunossupresive therapy should be attempted in subjects under suspition for AIH (Class IIa). Treatment should be evaluated after 5 to 7 days and may not postpone LT, when indicated (Class IIb)

- Subjects submitted to LT for AIH should receive higher immunossupression, but there is no consensus about the requirement of corticosteroids indefinitively in the long-term (Class IIa).

- Protocol liver biopsies may increase the diagnosis of AIH recurrence after LT in patients witohut clinical and biochemical signs of liver disease. The is no stablish benefit of treatment in this setting (Class IIb). The role of protocol liver biopsies in PBC and PSC is even less clear and they should not be performed (Class IIb).

Bittencourt PL, Cançado ELR, Couto CA, Levy C, Porta G, Silva AEB, Terrabuio DRB, Membros da $1^{\text {a }}$ Reunião de Consenso da Sociedade Brasileira de Hepatologia sobre Diagnóstico e Manejo das Doenças Auto-imunes do Fígado. Recomendações da Sociedade Brasileira de Hepatologia para diagnósticos e tratamento de doenças auto-imunes do fígado. Arq Gastroenterol. 2015(Supl 1):15-46.

RESUMO - Para definir as recomendações baseadas em evidências científicas sobre o diagnóstico e tratamento das doenças autoimnus do fígado, a Sociedade Brasileira de Hepatologia organizou em Outubro de 2014, encontro monotemático em São Paulo. Um Comitê organizador de sete investigadores foi selecionado pela Diretoria da Sociedade para organizar a agenda científica, assim como para selecionar vinte debatedores para fazer uma revisão sistemática e apresentar tópicos relacionados à hepatite autoimune, colangite esclerosante primária, cirrose biliar primária e suas síndromes de superposição (overlap). O texto inicial do submetidoo a apreciação e aprovação da Sociedade Brasileira de Hepatologia através de consulta a todos associados através da home page da Sociedade, O trabalho apresentado representa a versão final do trabalho original, devidamente revisado e organizado em tópicos, segundo as recomendações da Sociedade Brasileira de Hepatologia.

DESCRITORES - Hepatite autoimune. Colangite esclerosante primária. Cirrose biliar primária. Diagnóstico. Tratamento. 


\section{REFERENCES}

1. Methodology Manual for ACC/AHA Guideline Writing Committees: Methodologies and Policies from the ACC/AHA Task Force on Practice Guidelines April 2006. 2006.

2. Shiffman RN, Shekelle P, Overhage JM, Slutsky J, Grimshaw J, Deshpande AM. Standardized reporting of clinical practice guidelines: a proposal from the Conference on Guideline Standardization. Ann Intern Med 2003;139:493-498.

3. MacFarlane IG. Autoimmune hepatitis: diagnostic criteria, subclassifications, and clinical features. Clin Liver Dis 2002;6:605-21.

4. Thiele DL. Autoimmune hepatitis. Clin Liver Dis 2005;9:635-46.

5. Czaja AJ, Souto EO, Bittencourt PL, Cancado ELR, Porta G, Goldberg AC, Donaldson PT. Clinical distinctions and pathogenic implications of type 1 autoimmune hepatitis in Brazil and the United States. J Hepatol 2002;37:302-8.

6. Terrabuio DBR. 20 anos de hepatite autoimune. HCFMUSP. Dissertação de mestrado. FMUSP. 2006

7. Porta G. Autoimmune hepatitis. J Pediatr (Rio J). 2000;76 Suppl 1:S181-6.

8. Gregorio GV, Portmann B, Karani J, Harrison P, Donaldson PT, Vergani D, Mieli-Vergani G. Autoimmune hepatitis/sclerosing cholangitis overlap syndrome in childhood: a 16-year prospective study. Hepatology.2001;33:544-53.

9. Czaja AJ. Acute and acute severe (fulminant) autoimune hepatitis. Dig Dis Sci 2013;58:897-914

10. Bittencourt PL, Farias AQ, Porta G, Cançado EL, Miura I, Pugliese R, Kalil J, Goldberg AC, Carrilho FJ. Frequency of concurrent autoimmune disorders in patients with autoimmune hepatitis. Effect of Age, Gender, and Genetic Background. J Clin Gastroenterol 2008;42:300-5.

11. Manns MP, Czaja AJ, Gorham JD, et al. Practice Guidelines of the American Association for the Study of Liver Diseases. Diagnosis and management of autoimmune hepatitis. Hepatology 2010;51:2193-213.

12. Alvarez F, Berg PA, Bianchi FB, Bianchi L, Burroughs AK, Cancado EL, et al. International Autoimmune Hepatitis Group Report: review of criteria for diagnosis of autoimmune hepatitis. J Hepatol 1999;31:929-38.

13. Gleeson D, Heneghan MA. British Society of Gastroenterology (BSG) guidelines for management of autoimmune hepatitis. Gut 2011;60:1611-29.

14. Czaja. The role of autoantibodies as diagnostic markers of autoimmune hepatitis. Expert Rev Clin Immunol 2006:2:33-48.

15. Vergani D, Alvarez F, Bianchi FB, Cancado EL, Mackay IR, Manns MP, Nishioka $\mathrm{M}$, et al. Liver autoimmune serology: a consensus statement from the committee for autoimmune serology of the International Autoimmune Hepatitis Group. J Hepatol 2004;41:677-83.

16. Couto CA, Bittencourt PL, Porta G, Abrantes-Lemos CP, Carrilho FJ, Guardia BD, Cançado EL. Antismooth muscle and antiactin antibodies are indirect markers of histological and biochemical activity of autoimmune hepatitis. Hepatology. 2014 59:592-600.

17. Lohse A, Mieli-Vergani G. Autoimmune hepatitis. J Hepatol 2011;55:171-182.

18. Dellavance A, Gabriel-Jr A, Nuccitelli B, Taliberti BH, von Mühlen CA, Bichara CDA et al. Terceiro Consenso Brasileiro para pesquisa de autoanticorpos em Células HEp-2 (FAN) Third Brazilian Consensus for autoantibodies screening in HEp-2 cells (ANA) Rev Bras Reumatol 2009;49:89-109.

19. Bottazzo GF, Florin-Christensen A, Fairfax A, Swana G, Doniach D, Groeschel-Stewart U. Classification of smooth muscle autoantibodies detected by immunofluorescence. J Clin Pathol 1976;29:403-10.

20. Cançado EL, Abrantes-Lemos CP, Vilas-Boas LS, Novo NF, Carrilho FJ, Laudanna AA. Thermolabile and calcium-dependent serum factor interferes with polymerized actin, and impairs anti-actin antibody detection. J Autoimmun. 2001; 17:223-8

21. Rizzetto M, Bianchi FB, Doniach D.Characterization of the microsomal antigen related to a subclass of active chronic hepatitis. Immunology 1974;26:589-601.

22. Homberg JC, Abuaf N, Bernard O, Islam S, Alvarez F, Khalil SH, Poupon R, Darnis F, Lévy VG, Grippon P, et al. Chronic active hepatitis associated with antiliver/kidney microsome antibody type 1: a second type of "autoimmune" hepatitis. Hepatology 1987;7:1333-9.

23. Seelig R, Renz M, Bünger G, Schröter H, Seelig HP. Anti-LKM-1 antibodies determined by use of recombinant P450 2D6 in ELISA and western blot and their association with anti-HCV and HCV-RNA. Clin Exp Immunol 1993;92:373-80.

24. Bittencourt PL, Goldberg AC, Cançado EL, Porta G, Carrilho FJ, Farias AQ Palacios SA, Chiarella JM, Abrantes-Lemos CP, Baggio VL, Laudanna AA, Kali J. Genetic heterogeneity in susceptibility to autoimmune hepatitis types 1 and 2 . Am J Gastroenterol. 1999;94:1906-13.

25. Djilali-Saiah I, Fakhfakh A, Louafi H, Caillat-Zucman S, Debray D, Alvarez F. HLA class II influences humoral autoimmunity in patients with type 2 autoimmune hepatitis. J Hepatol. 2006;45:844-50.
26. Costa M, Rodríguez-Sánchez JL, Czaja AJ, Gelpí C. Isolation and characterization of cDNA encoding the antigenic protein of the human tRNP(Ser)Sec complex recognized by autoantibodies from patients withtype-1 autoimmune hepatitis. Clin Exp Immunol. 2000 Aug;121(2):364-74

27. Palioura S, Herkel J, Simonovi冈 M, Lohse AW, Söll D. Human SepSecS or SLA/LP: selenocysteine formation and autoimmune hepatitis. Biol Chem 2010;391:771-6.

28. Baeres M, Herkel J, Czaja AJ, Wies I, Kanzler S, Cancado EL, Porta G, Nishioka M, Simon T, Daehnrich C, Schlumberger W, Galle PR, Lohse AW. Establishment of standardised SLA/LP immunoassays: specificity for autoimmune hepatitis, worldwide occurrence, and clinical characteristics. Gut 2002;51:259-64.

29. Czaja A J, Autoantibodies as prognostic Markers in Autoimmune Liver Disease. Dig Dis Sci 2010;55:2144-61.

30. Martini E, Abuaf N, Cavalli F, Durand V, Johanet C, Homberg JC. Antibody to liver cytosol (anti-LC1) in patients with autoimmune chronic active hepatitis type 2. Hepatology 1988;8:1662-6.

31. Johanet C, Ballot E. Autoantibodies in autoimmune hepatitis: anti-liver kidney microsome type 1 (anti-LKM1) and anti-liver cytosol type 1 (anti-LC1) antibodies. Clin Res Hepatol Gastroenterol 2013;37:216-8.

32. Liberal R, Mieli-Vergani G, Vergani D. Clinical significance of autoantibodies in autoimmune hepatitis. J Autoimmun 2013;46:17-24.

33. Hennes $\mathrm{EM}^{1}$, Zeniya M, Czaja AJ, Parés A, Dalekos GN, Krawitt EL, Bittencourt PL, Porta G, Boberg KM, Hofer H, Bianchi FB, Shibata M, Schramm C, Eisenmann de Torres B, Galle PR, McFarlane I, Dienes HP, Lohse AW; International Autoimmune Hepatitis Group. International Autoimmune Hepatitis Group. Simplified criteria for the diagnosis of autoimmune hepatitis. Hepatology 2008;48:169-76

34. Czaja A. Perfomance of Diagnostic Scoring Systems for Autoimmune Hepatitis. Hepatology 2008;48:1540-48.

35. Abe K, Kanno Y, Okai K, Katsushima F, Monoe K, Saito H, Takahashi A, Yokokawa J, Ohira H. Centrilobular necrosis in acute presentation of Japanese patients with type 1 autoimmune hepatitis. World J Hepatol. 2012 Sep 27;4:262-7.

36. Stravitz RT, Lefkowitch JH, Fontana RJ, , Gershwin ME, Leung PS, Sterling RK, Manns MP, Norman GL, Lee WM; Acute Liver Failure Study Group. Autoimmune acute liver failure: proposed clinical and histological criteria. Hepatology. 2011;53:517-26.

37. Cançado ELR, Porta G. Autoimmune hepatitis in South America. In: Manns MP, Paumgartner G, Leuschner U. Immunology and Liver. Falk Symposium 114. Dordrecht: Kluwer Academic Publishers, 2000;82-92.

38. Czaja AJ, Lindor KD. Failure of budesonide in a pilot study of treatment-dependent autoimmune hepatitis. Gastroenterology 2000; 119:1312-6

39. Manns MP, Woynarowski M, Kreisel W, Lurie Y, Rust C, Zuckerman E, Bahr MJ, Günther R, Hultcrantz RW, Spengler U, Lohse AW, Szalay F, Färkkilä M, Pröls M, Strassburg CP; European AIH-BUC-Study Group. Budesonide induces remission more effectively than prednisone in a controlled trial of patients with autoimmune hepatitis. Gastroenterology 2010;139:1198-206.

40. Dubinsky MC, Lamothe S, Yang HY, Targan SR, Sinnett D, Théorêt Y, Seidman EG. Pharmacogenomics and metabolite measurement for 6-mercaptopurine therapy in inflammatory bowel disease. Gastroenterology 2000;118:705-13.

41. Sparrow MP, Hande SA, Friedman S, Lim WC, Reddy SI, Cao D, Hanauer SB Allopurinol safely and effectively optimizes tioguanine metabolites in inflammatory bowel disease patients not responding to azathioprine and mercaptopurine. Aliment Pharmacol Ther 2005;22:441-6.

42. de Boer YS, van Gerven NM, de Boer NK, Mulder CJ, Bouma G, van Nieuwkerk CM. Allopurinol safely and effectively optimises thiopurine metabolites in patients with autoimmune hepatitis. Aliment Pharmacol Ther 2013 Mar;37:640-6.

43. Czaja AJ, Menon KV, Carpenter HA. Sustained remission after corticosteroid therapy for type 1 autoimmune hepatitis: a retrospective analysis. Hepatology. 2002;35:890-7

44. Mucenic M, Mello ES, Cançado EL. Chloroquine for the maintenance of remission of autoimmune hepatitis: results of a pilot study. Arq Gastroenterol 2005; $42: 249-55$.

45. Heneghan MA, Norris SM, O'Grady JG, Harrison PM, McFarlane IG. Management and outcome of pregnancy in autoimmune hepatitis. Gut. 2001;48:97-102.

46. Terrabuio DR, Abrantes-Lemos CP, Carrilho FJ, Cançado EL. Follow-up of pregnant women with autoimmune hepatitis: the disease behavior along with maternal and fetal outcomes. J Clin Gastroenterol 2009;43:350-6.

47. López-Méndez E, Avila-Escobedo L. Pregnancy and portal hypertension a pathology view of physiologic changes. Ann Hepatol.2006;5:219-23.

48. Selvarajah V, Montano-Loza AJ, Czaja AJ. Systematic review: managing suboptimal treatment responses in autoimmune hepatitis with conventional and nonstandard drugs. Aliment Pharmacol Ther 2012;36:691-707. 
49. Jothimani D, Cramp ME, Mitchell JD, Cross TJS. Treatment of autoimmune hepatitis: A review of current and evolving therapies. J Gastroenterol Hepatol 2011;26:619-27.

50. Czaja AJ. Advances in the Current Treatment of Autoimmune Hepatitis. Dig Dis Sci 2012;57:1996-2010.

51. Moura MC, Liberal R, Cardoso H, Horta e Vale AM, Macedo G. Management of autoimmune hepatitis: Focus on pharmacologic treatments beyond corticosteroids. World J Hepatol 2014;6:410-418.

52. Czaja AJ. Autoimmune hepatitis: focusing on treatments other than steroids. Can J Gastroenterol 2012;26:615-20.

53. Malekzadeh R, Nasseri-Moghaddam S, Kaviani MJ, Taheri H, Kamalian N, Sotoudeh . Cyclosporin A is a promising alternative to corticosteroids in autoimmune hepatitis. Dig Dis Sci 2001;46:1321-1327.

54. Zachou K, Gatselis N, Papadamou G, Rigopoulou EI, Dalekos GN. Mycophenolate for the treatment of autoimmune hepatitis: prospective assessment of its efficacy and safety for induction and maintenance of remission in a large cohort of treatment-naive patients. J Hepatol 2011;55:636-46.

55. Burak KW, Swain MG, Santodomino-Garzon T, Lee SS, Urbanski SJ, Aspinall AI, Coffin CS, Myers RP. Rituximab for the treatment of patients with autoimmune hepatitis who are refractory or intolerante to standard therapy. Can J Gastroenterol 2013;27:273-80.

56. Weiler-Normann C, Schramm C, Quaas A, Wiegard C, Glaubke C, Pannicke N, Moller S, Lohse AW. Infliximab as a rescue treatment in difficult to treat autoimmune hepatitis. J Hepatol 2013;58:529-34.

57. Nakamura K, Yoneda M, Yokohama S, Tamori K, Sato Y, Aso K, Aoshima M, Hasegawa T, Makino I. Efficacy of ursodeoxycholic acid in Japanese patients with type 1 autoimmune hepatitis. J Gastroenterol Hepatol 1998;13:490-5.

58. Czaja AJ, Carpenter HA, Lindor KD. Ursodeoxycholic acid as adjunctive therapy for problematic type 1 autoimmune hepatitis: a randomized placebo-controlled treatment trial. Hepatology 1999;30:1381-6.

59. Vergani D, Mieli-Vergani G. Pharmacological management of autoimmune hepatitis. Expert Opin Pharmacother 2011;12:607-13.

60. Woynarowski M, Nemeth, A, Baruch Y, Koletzko S, Melter M, Rodeck B, Strassburg CP, Pröls M, Wo囚niak M, Manns MP; European Autoimmune Hepatitis-Budesonide Study Group. Budesonide versus Prednisone with Azathioprine for the Treatment of Autoimmune Hepatitis in Children and Adolescents. J Pediatr 2013;163:1347-53.

61. Alvarez F, Ciocca M, Canero-Velasco C, Ramonet M de Davila MT, Cuarterolo M, Gonzalez T, Jara-Vega P, Camarena C, Brochu P, Drut R, Alvarez E. Shortterm cyclosporine induces a remission of autoimmune hepatitis in children. J Hepatol 1999;30:222-7.

62. Marlaka JR, Papadogiannakis N, Fischler B, Casswall TH, Beijer E, Nemeth A. Tacrolimus without or with the addition of conventional immunosuppressive treatment in juvenile autoimmune hepatitis. Acta Paediatr 2012;101:993-9.

63. Aw MM, Dhawan A, Samyn M, Bargiota A, Mielli-Vergani G. Mycophenolate mofetil as rescue treatment for autoimmune liver disease in children: a 5-year follow-up. J Hepatol 2009;51:156-60.

64. D'Agostino D, Costaguta A and Alvarez F. Successful Treatment of Refractory Autoimmune Hepatitis With Rituximab. Pediatrics 2013;132:526-530.

65. Czaja AJ. Review article: the management of autoimmune hepatitis beyond consensus guidelines. Aliment Pharmacol Ther 2013;38:343-64.

66. Maggs JR, Chapman RW. An update on primary sclerosing cholangitis. Curr Opin Gastroenterol 2008; 24: 377-83.

67. Karlsen TH, Schrumpf E, Boberg KM. Genetic epidemiology of primary sclerosing cholangitis. World J Gastroenterol 2007; 13: 5421-31.

68. European Association for the Study of the Liver EASL Clinical Practice Guidelines: Management of cholestatic liver diseases. Journal of Hepatology 2009; 51: $237-67$

69. Broome U, Olsson R, Loof L, Bodemar G, Hultcrantz R, Danielsson A, et al Natural history and prognostic factors in 305 Swedish patients with primary sclerosing cholangitis. Gut 1996; 38: 610-15.

70. Hirschfield GM, Karlsen TH, Lindor KD, Adams DH. Primary sclerosing cholangitis. Lancet 2013; 382: 1587-99.

71. Nguyen DL, LaRusso NF, Lazaridis KN. In Schiff ER, Maddrey WC, Sorrell MF, eds. Schiff's Diseases of the Liver, $11^{\text {th }}$ edition. Cp. 20, pgs 477-88.

72. Angulo P, Maor-Kendler Y, Lindor KD. Small-duct primary sclerosing cholangitis: a long term follow-up study. Hepatology 2002; 35: 1494-1500.

73. Nishimori I, Otsuki M. Autoimmune pancreatitis and IgG4-associated sclerosing cholangitis. Best Practice \& Research Clinical Gastroenterology 2009; 23: 11-23.

74. Kamisawa T, Okamoto A. IgG4-related sclerosing disease. World J Gastroenterol 2008; 14: 3948-55.

75. Chapman R, Fevery J, Kalloo A, Nagorney DM, Boberg KM, Shneider B, and Gores GJ. Diagnosis and management of primary sclerosing cholangitis. Hepatology 2010; 51: 660-78.
76. Chapman RW, Arborgh BA, Rhodes JM, Summerfield JA, Dick R, Scheuer PJ, et al. Primary sclerosing cholangitis: a review of its clinical features, cholangiography, and hepatic histology. Gut 1980; 21: 870-877.

77. Abdalain R \& Heathcote EJ. Sclerosing cholangitis: a focus on secondary causes. Hepatology 2006; 44: 1063-74.

78. Burak KW, Angulo P, Lindor KD. Is there a role for liver biopsy in primary sclerosing cholangitis? Am J Gastroenterol 2003; 98: 1155-8.

79. Chazouillères O, Poupon R, Capron JP, Metman EH, Dhumeaux D, Amouretti M, Couzigou P, Labayle D, Trinchet JC. Ursodeoxycholic acid for primary sclerosing cholangitis. J Hepatol 1990; 11(1): 120-3.

80. Poupon R. Ursodeoxycholic acid and bile-acid mimetics as therapeutic agents for cholestatic liver diseases: an overview of their mechanisms of action. Clin Res Hepatol Gastroenterol 2012; 36 (Suppl 1): S3-12.

81. Shi J, Li Z, Zeng X, Lin Y, Xie WF. Ursodeoxycholic acid in primary sclerosing cholangitis: meta-analysis of randomized controlled trials. Hepatol Res 2009; 39(9) 865-73

82. Triantos CK, Koukias NM, Nikolopoulou VN, Burroughs AK. Meta-analysis: ursodeoxycholic acid for primary sclerosing cholangitis. Aliment Pharmacol Ther 2011; 34 (8): 901-10.

83. Poropat G, Giljaca V, Stimac D, Gluud C. Bile acids for primary sclerosing cholangitis. Cochrane Database Syst Rev 2011; (1): CD003626.

84. Stanich PP, Björnsson E, Gossard AA, Enders F, Jorgensen R, Lindor KD. Alkaline phosphatase normalization is associated with better prognosis in primary sclerosing cholangitis. Dig Liver Dis 2011; 43(4): 309-13.

85. Al Mamari S, Djordjevic J, Halliday JS, Chapman RW. Improvement of serum alkaline phosphatase to $<1.5$ upper limit of normal predicts better outcome and reduced risk of cholangiocarcinoma in primary sclerosing cholangitis. J Hepatol 2013; 58(2): 329-34.

86. Lindström L, Hultcrantz R, Boberg KM, Friis-Liby I, Bergquist A. Association between reduced levels of alkaline phosphatase and survival times of patients with primary sclerosing cholangitis. Clin Gastroenterol Hepatol 2013 11(7): 841-6.

87. Wunsch E, Trottier J, Milkiewicz M, Raszeja-Wyszomirska J, Hirschfield GM, Barbier O, Milkiewicz P. Prospective evaluation of ursodeoxycholic acid withdrawal in patients with primary sclerosing cholangitis. Hepatology 2014; 60(3): $931-40$

88. Mizuno S, Hirano K, Tada M, Yamamoto K, Yashima Y, Yagioka H, Kawakubo K, Ito Y, Kogure H, Sasaki T, Arizumi T, Togawa O, Matsubara S, Nakai Y, Sasahira N, Tsujino T, Isayama H, Kawabe T, Omata M, Koike K. Bezafibrate for the treatment of primary sclerosing cholangitis. J Gastroenterol 2010 , 45(7): 758-62

89. Kita R, Kita-Sasai Y, Hanaoka I, Kimura T, Kokuryu H, Takamatsu S, Osaki Y Tomono N, Hachiya T, Shimizu T. Beneficial effect of bezafibrate on primary sclerosing cholangitis (three case reports). Am J Gastroenterol 2002; 97(7): 1849-51.

90. Lindor KD, Kowdley KV, Luketic VA, Harrison ME, McCashland T, Befeler AS, Harnois D, Jorgensen R, Petz J, Keach J, Mooney J, Sargeant C, Braaten J, Bernard T, King D, Miceli E, Schmoll J, Hoskin T, Thapa P, Enders F. Highdose ursodeoxycholic acid for the treatment of primary sclerosing cholangitis. Hepatology 2009; 50(3):808-14.

91. Olsson R, Boberg KM, de Muckadell OS, Lindgren S, Hultcrantz R, Folvik G Bell H, Gangsøy-Kristiansen M, Matre J, Rydning A, Wikman O, Danielsson A, Sandberg-Gertzén H, Ung KA, Eriksson A, Lööf L, Prytz H, Marschall HU, Broomé U. High-dose ursodeoxycholic acid in primary sclerosing cholangitis: a 5-year multicenter, randomized, controlled study. Gastroenterology 2005; 129(5): 1464-72.

92. Tung BY, Emond MJ, Haggitt RC, Bronner MP, Kimmey MB, Kowdley KV, Brentnall TA. Ursodiol use is associated with lower prevalence of colonic neoplasia in patients with ulcerative colitis and primary sclerosing cholangitis. Ann Intern Med 2001; 134(2): 89-95.

93. Pardi DS, Loftus EV Jr, Kremers WK, Keach J, Lindor KD. Ursodeoxycholic acid as a chemopreventive agent in patients with ulcerative colitis and primary sclerosing cholangitis. Gastroenterology 2003; 124(4): 889-93.

94. Wolf JM, Rybicki LA, Lashner BA. The impact of ursodeoxycholic acid on can cer, dysplasia and mortality in ulcerative colitis patients with primary sclerosing cholangitis. Aliment Pharmacol Ther 2005; 22(9): 783-8.

95. Lindström L, Boberg KM, Wikman O, Friis-Liby I, Hultcrantz R, Prytz H, Sandberg-Gertzén H, Sangfelt P, Rydning A, Folvik G, Gangsøy-Kristiansen M, Danielsson A, Bergquist A. High dose ursodeoxycholic acid in primary sclerosing cholangitis does not prevent colorectal neoplasia. Aliment Pharmacol Ther 2012; 35(4): 451-7.

96. Braden B, Halliday J, Aryasingha S, Sharifi Y, Checchin D, Warren BF, Kitiyakara T, Travis SP, Chapman RW. Risk for colorectal neoplasia in patients with colonic Crohn's disease and concomitant primary sclerosing cholangitis. Clin Gastroenterol Hepatol 2012; 10(3): 303-8 
97. Navaneethan U, Kochhar G, Venkatesh PG, Lewis B, Lashner BA, Remzi FH, Shen B, Kiran RP. Duration and severity of primary sclerosing cholangitis is not associated with risk of neoplastic changes in the colon in patients with ulcerative colitis. Gastrointest Endosc 2012; 75(5): 1045-1054.e1.

98. Eaton JE, Silveira MG, Pardi DS, Sinakos E, Kowdley KV, Luketic VA, Harrison ME, McCashland T, Befeler AS, Harnois D, Jorgensen R, Petz J, Lindor KD. High-dose ursodeoxycholic acid is associated with the development of colorectal neoplasia in patients with ulcerative colitis and primary sclerosing cholangitis. Am J Gastroenterol 2011; 106(9): 1638-45.

99. Hansen JD, Kumar S, Lo WK, Poulsen DM, Halai UA, Tater KC. Ursodiol and colorectal cancer or dysplasia risk in primary sclerosing cholangitis and inflammatory bowel disease: a meta-analysis. Dig Dis Sci 2013; 58(11):3079-87.

100. Janczewska I, Olsson R, Hultcrantz R, Broomé U. Pregnancy in patients with primary sclerosing cholangitis. Liver 1996; 16(5): 326-30.

101. Wellge BE, Sterneck M, Teufel A, Rust C, Franke A, Schreiber S, Berg T, Günther R, Kreisel W, Zu Eulenburg C, Braun F, Beuers U, Galle PR, Lohse AW, Schramm C. Pregnancy in primary sclerosing cholangitis. Gut 2011; 60(8): 1117-21.

102. Ludvigsson JF, Bergquist A, Ajne G, Kane S, Ekbom A, Stephansson O. A population-based cohort study of pregnancy outcomes among women with primary sclerosing cholangitis. Clin Gastroenterol Hepatol 2014; 12(1): 95-100.e1.

103. Kammeijer CQ, De Man RA, De Groot CJ. Primary sclerosing cholangitis and pregnancy. Clin Pract 2011; 1(3): e55.

104. Brites D, Rodrigues CM. Elevated levels of bile acids in colostrum of patients with cholestasis of pregnancy are decreased following ursodeoxycholic acid therapy. J Hepatol 1998; 29(5): 743-51.

105. Vítek L, Zelenková M, Brůha R. Safe use of ursodeoxycholic acid in a breast-feeding patient with primary biliary cirrhosis. Dig Liver Dis 2010; 42(12): 911-2.

106. Aljiffry M, Renfrew P, Molinari M et al. Analytical review of diagnosis and treatment strategies for dominant bile duct strictures in patients with primary sclerosing cholangitis. HPB, 2011; 13: 79-90.

107. Stiehl A, Rudolph G, Kloters-Plachky P et al. Development of dominant bile duct stenosis in patients with primary sclerosing cholangitis treated with ursodeoxycholic acid: outcome after endoscopic treatment. Journal of Hepatology. 2002; 36: 151-6.

108. Baluyut AR, Sherman S, Lehman GA ET AL. Impact of endoscopic therapy on the survival of patients with primary sclerosing cholangitis. Gastrointestinal endosc. 2001; 53: 308-312.

109. Ismail S, Kylanpaa L, Mustonen $\mathrm{H}$ et al. Risk factors for complications of CPRE in primary sclerosing cholangitis. Endoscopy. 2012; 44:1133-8.

110. Alkhatib AA, Hilden K, Adler Dg. Comorbidities, sphincterotomy, and ballon dilation predict post-CPRE adverse events in PSC patients: operator experience is protective. Digestive diseases and sciences. 2011;56:3685-8.

111. Kaya M, Petersen BT Baron TH at al. Ballon dilation compared to stenting of dominant strictures in primary sclerosing cholangitis. The American journal of gastroenterology. 2001;96: 1059-66.

112. Ponsioen CY, Lam K, Tytgat GN. Four years experience with short-term stenting in primary sclerosing cholangitis. Am J Gastroenterol. 1999;94:2403-2407.

113. Shneider BL. Diagnostic and therapeutic challenges in pediatric primary sclerosing cholangitis. Liver Transplant 2012; 18: 277-281.

114. Miethke AG, Balistreri WF. (2014) Sclerosing cholangitis In: Suchy FJ, Sokol RJ, Balsitrei WT (eds). Liver disease in children, 4th edition, Cambridge University, USA, pp322-340.

115. Kerkar N, Miloh T. Sclerosing cholangitis: pediatric perspective. Curr Gastroenterol Rep 2010; 12: 195-202

116. Mieli-Vergani G, Vergani D. Unique features of primary sclerosing cholangitis in children. Curr Opin Gastroenterol 2010: 26: 265-268.

117. Ibrahim SH, Lindor KD. Current management of primary sclerosing cholangiris in pediatric patients. Pediatr Drugs 2011; 13 (2): 87-95.

118. Girard M, Franchi-Abella S, Lacaille F, Debray D. Specificities of sclerosing cholangitis in childhood. Cin Res Hepatol Gastroenterol 2012; 36: 530-535.

119. Gilger MA, Gann ME, Pelun AR et al. Efficacy of ursodeoxycholic acid in the treatment of primary sclerosing cholangitis in childrem.

120. Abarbanel DN, Seki SM, Davies Y et al. Immunomodulatory effect of vancomycin or Treg in pediatric inflammatory bowel disease and primary sclerosing cholangitis. J Clin Immunol 2013: 33: 397-406

121. Lindor KD, Gershwin ME, Poupon R, Kaplan M, Bergasa NV, Heathcote EJ, et al. Primary biliary cirrhosis. Hepatology. 2009;50(1):291-308

122. Francescantonio PL, Cruvinel Wde M, Dellavance A, Andrade LE, Taliberti BH, von Muhlen CA, et al. IV Brazilian guidelines for autoantibodies on HEp-2 cells. Revista brasileira de reumatologia. 2014;54(1):44-50.

123. Nakamura M. Clinical significance of autoantibodies in primary biliary cirrhosis Seminars in liver disease. 2014;34(3):334-40.
124. Andrejevic S, Bonaci-Nikolic B, Sefik-Bukilica M, Petrovic R. Clinical and serological follow-up of 71 patients with anti-mitochondrial type 5 antibodies. Lupus. 2007;16(10):788-93.

125. Chantran Y, Ballot E, Johanet C. Autoantibodies in primary biliary cirrhosis: antimitochondrial autoantibodies. Clinics and research in hepatology and gastroenterology. 2013;37(4):431-3

126. Miyakawa H, Tanaka A, Selmi C, Hosoya N, Mataki N, Kikuchi K, et al. Serum reactivity against bacterial pyruvate dehydrogenase: increasing the specificity of anti-mitochondrial antibodies for the diagnosis of primary biliary cirrhosis Clinical \& developmental immunology. 2006;13(2-4):289-94.

127. Granito A, Muratori P, Quarneti C, Pappas G, Cicola R, Muratori L. Antinuclea antibodies as ancillary markers in primary biliary cirrhosis. Expert review of molecular diagnostics. 2012;12(1):65-74.

128. Poupon R. Primary biliary cirrhosis: a 2010 update. J Hepatol. 2010;52(5):745-58

129. Trivedi PJ, Bruns T, Cheung A, Li KK, Kittler C, Kumagi T, et al. Optimising risk stratification in primary biliary cirrhosis: AST/platelet ratio index predicts outcome independent of ursodeoxycholic acid response. J Hepatol. 2014;60(6):1249-58.

130. Corpechot C, Carrat F, Poujol-Robert A, Gaouar F, Wendum D, Chazouilleres $\mathrm{O}$, et al. Noninvasive elastography-based assessment of liver fibrosis progression and prognosis in primary biliary cirrhosis. Hepatology. 2012;56(1):198-208.

131. Zhang DK, Chen M, Liu Y, Wang RF, Liu LP, Li M. Acoustic radiation force impulse elastography for non-invasive assessment of disease stage in patients with primary biliary cirrhosis: A preliminary study. Clinical radiology. 2014;69(8):836-40

132. Patanwala I, McMeekin P, Walters R, Mells G, Alexander G, Newton J, et al. A validated clinical tool for the prediction of varices in PBC: the Newcastle Varices in PBC Score. J Hepatol. 2013;59(2):327-35.

133. Levy C, Zein CO, Gomez J, Soldevila-Pico C, Firpi R, Morelli G, et al. Prevalence and predictors of esophageal varices in patients with primary biliary cirrhosis. Clinical gastroenterology and hepatology : the official clinical practice journal of the American Gastroenterological Association. 2007;5(7):803-8.

134. Al-Harthy N, Kumagi T. Natural history and management of primary biliary cirrhosis. Hepatic medicine : evidence and research. 2012;4:61-71.

135. Quarneti C, Muratori P, Lalanne C, Fabbri A, Menichella R, Granito A, et al. Fatigue and pruritus at onset identify a more aggressive subset of primary biliary cirrhosis. Liver international : official journal of the International Association for the Study of the Liver. 2014.

136. Nakamura M, Kondo H, Mori T, Komori A, Matsuyama M, Ito M, et al. Anti-gp210 and anti-centromere antibodies are different risk factors for the progression of primary biliary cirrhosis. Hepatology. 2007;45(1):118-27.

137. Hirschfield GM, Gershwin ME. Primary biliary cirrhosis: one disease with many faces. The Israel Medical Association journal : IMAJ. 2011;13(1):55-9.

138. Czaja AJ. Diagnosis and management of the overlap syndromes of autoimmune hepatitis. Canadian journal of gastroenterology = Journal canadien de gastroenterologie. 2013;27(7):417-23.

139. Floreani A, Franceschet I, Cazzagon N. Primary biliary cirrhosis: overlaps with other autoimmune disorders. Seminars in liver disease. 2014;34(3):352-60.

140. Bittencourt PL, Farias AQ, Abrantes-Lemos CP, Goncalves LL, Goncalves PL, Magalhaes EP, et al. Prevalence of immune disturbances and chronic liver disease in family members of patients with primary biliary cirrhosis. Journal of gastroenterology and hepatology. 2004;19(8):873-8.

141. Mantaka A, Koulentaki M, Chlouverakis G, Enele-Melono JM, Darivianaki A, Tzardi M, et al. Primary biliary cirrhosis in a genetically homogeneous population: disease associations and familial occurrence rates. BMC gastroenterology. 2012:12:110

142. Lazaridis KN, Juran BD, Boe GM, Slusser JP, de Andrade M, Homburger HA, et al. Increased prevalence of antimitochondrial antibodies in first-degree relatives of patients with primary biliary cirrhosis. Hepatology. 2007;46(3):785-92.

143. Corpechot C, Abenavoli L, Rabahi N, Chretien Y, Andreani T, Johanet C, et al. Biochemical response to ursodeoxycholic acid and long-term prognosis in primary biliary cirrhosis. Hepatology. 2008;48(3):871-7.

144. Gong Y, Huang ZB, Christensen E, Gluud C. Ursodeoxycholic acid for primary biliary cirrhosis. The Cochrane database of systematic reviews. 2008(3):CD000551.

145. Poupon RE, Lindor KD, Cauch-Dudek K, Dickson ER, Poupon R, Heathcote EJ. Combined analysis of randomized controlled trials of ursodeoxycholic acid in primary biliary cirrhosis. Gastroenterology. 1997;113(3):884-90

146. Lindor KD, Jorgensen RA, Therneau TM, Malinchoc M, Dickson ER. Ursodeoxycholic acid delays the onset of esophageal varices in primary biliary cirrhosis. Mayo Clinic proceedings. 1997;72(12):1137-40.

147. Corpechot C, Carrat F, Bahr A, Chretien Y, Poupon RE, Poupon R. The effect of ursodeoxycholic acid therapy on the natural course of primary biliary cirrhosis. Gastroenterology. 2005;128(2):297-303.

148. Pares A, Caballeria L, Rodes J. Excellent long-term survival in patients with primary biliary cirrhosis and biochemical response to ursodeoxycholic Acid. Gastroenterology. 2006;130(3):715-20. 
149. Corpechot C, Chazouilleres O, Poupon R. Early primary biliary cirrhosis: biochemical response to treatment and prediction of long-term outcome. J Hepatol. 2011;55(6):1361-7.

150. Kuiper EM, Hansen BE, de Vries RA, den Ouden-Muller JW, van Ditzhuijsen TJ, Haagsma EB, et al. Improved prognosis of patients with primary biliary cirrhosis that have a biochemical response to ursodeoxycholic acid. Gastroenterology. 2009;136(4):1281-7.

151. Kumagi T, Guindi M, Fischer SE, Arenovich T, Abdalian R, Coltescu C, et al. Baseline ductopenia and treatment response predict long-term histological progression in primary biliary cirrhosis. Am J Gastroenterol. 2010;105(10):2186-94

152. Kuiper EM, Hansen BE, Metselaar HJ, de Man RA, Haagsma EB, van Hoek B, et al. Trends in liver transplantation for primary biliary cirrhosis in the Netherlands 1988-2008. BMC gastroenterology. 2010;10:144.

153. Lee J, Belanger A, Doucette JT, Stanca C, Friedman S, Bach N. Transplantation trends in primary biliary cirrhosis. Clinical gastroenterology and hepatology : the official clinical practice journal of the American Gastroenterological Association. 2007;5(11):1313-5

154. Rudic JS, Poropat G, Krstic MN, Bjelakovic G, Gluud C. Ursodeoxycholic acid for primary biliary cirrhosis. The Cochrane database of systematic reviews. 2012;12:CD000551.

155. Czul F, Peyton A, Levy C. Primary biliary cirrhosis: therapeutic advances. Clin Liver Dis. 2013;17(2):229-42.

156. Lammers WJ, van Buuren HR, Hirschfield GM, Janssen HL, Invernizzi P, Mason AL, et al. Levels of Alkaline Phosphatase and Bilirubin are Surrogate Endpoints of Outcomes of Patients with Primary Biliary Cirrhosis - an International Follow-up Study. Gastroenterology. 2014.

157. Trivedi PJ, Kumagi T, Al-Harthy N, Coltescu C, Ward S, Cheung A, et al. Good maternal and fetal outcomes for pregnant women with primary biliary cirrhosis. Clinical gastroenterology and hepatology : the official clinical practice journal of the American Gastroenterological Association. 2014;12(7):1179-85 e1.

158. Lammers WJ, van Buuren HR, Pares A, Hirschfield GM, Janssen HL, Kumagi $\mathrm{T}$, et al. Defining optimal laboratory response criteria in UDCA treated primary biliary cirrhosis. Results of an international multicenter long term follow-up study. Hepatology. 2013;58(4):249A

159. Leuschner M, Maier KP, Schlichting J, Strahl S, Herrmann G, Dahm HH, et al. Oral budesonide and ursodeoxycholic acid for treatment of primary biliary cirrhosis: results of a prospective double-blind trial. Gastroenterology. 1999;117(4):918-25.

160. Rautiainen H, Karkkainen P, Karvonen AL, Nurmi H, Pikkarainen P, Nuutinen $\mathrm{H}$, et al. Budesonide combined with UDCA to improve liver histology in primary biliary cirrhosis: a three-year randomized trial. Hepatology. 2005;41(4):747-52.

161. Iwasaki S, Akisawa N, Saibara T, Onishi S. Fibrate for treatment of primary biliary cirrhosis. Hepatol Res. 2007;37 Suppl 3:S515-7.

162. Levy C, Peter JA, Nelson DR, Keach J, Petz J, Cabrera R, et al. Pilot study: fenofibrate for patients with primary biliary cirrhosis and an incomplete response to ursodeoxycholic acid. Aliment Pharmacol Ther. 2011;33(2):235-42.

163. Honda A, Ikegami T, Nakamuta M, Miyazaki T, Iwamoto J, Hirayama T, et al. Anticholestatic effects of bezafibrate in patients with primary biliary cirrhosis treated with ursodeoxycholic acid. Hepatology. 2012.

164. Dohmen K, Mizuta T, Nakamuta M, Shimohashi N, Ishibashi H, Yamamoto K. Fenofibrate for patients with asymptomatic primary biliary cirrhosis. World journal of gastroenterology : WJG. 2004;10(6):894-8.

165. Ohira H, Sato Y, Ueno T, Sata M. Fenofibrate treatment in patients with primary biliary cirrhosis. Am J Gastroenterol. 2002;97(8):2147-9.

166. Nakamuta M, Enjoji M, Kotoh K, Shimohashi N, Tanabe Y. Long-term fibrate treatment for PBC. Journal of gastroenterology. 2005;40(5):546-7.

167. Han XF, Wang QX, Liu Y, You ZR, Bian ZL, Qiu de K, et al. Efficacy of fenofibrate in Chinese patients with primary biliary cirrhosis partially responding to ursodeoxycholic acid therapy. Journal of digestive diseases. 2012;13(4):219-24.

168. Walker LJ, Newton J, Jones DE, Bassendine MF. Comment on biochemical response to ursodeoxycholic acid and long-term prognosis in primary biliary cirrhosis. Hepatology. 2009;49(1):337-8; author reply 8 .

169. Iwasaki S, Ohira H, Nishiguchi S, Zeniya M, Kaneko S, Onji M, et al. The efficacy of ursodeoxycholic acid and bezafibrate combination therapy for primary biliary cirrhosis: A prospective, multicenter study. Hepatol Res. 2008;38(6):557-64.

170. Tanaka A, Hirohara J, Nakanuma Y, Tsubouchi H, Takikawa H. Biochemical responses to bezafibrate improve long-term outcome in asymptomatic patients with primary biliary cirrhosis refractory to UDCA. Journal of gastroenterology. 2014.

171. Rudic JS, Poropat G, Krstic MN, Bjelakovic G, Gluud C. Bezafibrate for primary biliary cirrhosis. The Cochrane database of systematic reviews. 2012;1:CD009145.

172. Grigorian AY, Mardini HE, Corpechot C, Poupon R, Levy C. Fenofibrate is effective adjunctive therapy in the treatment of primary biliary cirrhosis: A meta-analysis. Clin Res Hepatol Gastroenterol. 2015;39:296-306.
173. Komori A, nakamura M, Aiba Y, Kugiyama-Yasunaga Y, Bekki S, Sasaki R, et al. Who may have treatment benefits with fibrates in primary biliary cirrhosis: a single center retrospective observational cohort analysis. J Hepatol. 2013;58:S387.

174. Hirschfield GM, Mason A, Luketic V, Lindor K, Gordon SC, Mayo M, Kowdley KV, Vincent C, Bodhenheimer HC, Parés A, Trauner M, Marschall HU, Adorini L, Sciacca C, Beecher-Jones T, Castelloe E, Böhm O, Shapiro D. Efficacy of obeticholic Acid in patients with primary biliary cirrhosis and inadequate response to ursodeoxycholic Acid. Gastroenterology. 2015;148:751-61.

175. Couto CA; Bittencourt PL. Controvérsias no diagnóstico e tratamento da Hepatite Autoimune. In: Paulo Roberto Savassi Rocha; Luiz Gonzaga Vaz Coelho; Marcelo Sanchez. (Org.). Tópicos em Gastrenterologia XIII. Rio de Janeiro: MEDSI, 2004

176. Boberg KM, Chapman RW, Hirschfield GM, Lohse AW, Manns MP, Schrumpf E. Overlap syndromes: the International Autoimmune Hepatitis Group (IAIHG) position statement on a controversial issue. J Hepatol. 2011;54(2):374-385.

177. Bittencourt PL, Palacios SA, Cancado EL, Porta G, Carrilho FJ, Goldberg AC. Susceptibility to primary sclerosing cholangitis in Brazil is associated with HLA-DRB1*13 but not with tumor necrosis fator alpha -308 polymorphism. Gut 2002; 51: 609-610

178. Al-Chalabi T, Portmann BC, Bernal W, McFarlane IG, Heneghan MA. Autoimmune hepatitis overlap syndromes: an evaluation of treatment response, long-term outcome and survival. Aliment Pharmacol Ther. 2008;28:209-20.

179. Lewin M, Vilgrain V, Ozenne V, et al. Prevalence of sclerosing cholangitis in adults with autoimmune hepatitis: a prospective magnetic resonance imaging and histological study. Hepatology. 2009:50:528-537.

180. Abdalian R, Dhar P, Jhaveri K, AIHder M, Guindi M, Heathcote EJ. Prevalence of sclerosing cholangitis in adults with autoimmune hepatitis: evaluating the role of routine magnetic resonance imaging. Hepatology. 2008;47:949-57.

181. Culver EL, Chapman RW. Systematic review: management options for primary sclerosing cholangitis and its variant forms - IgG4-associated cholangitis and overlap with autoimmune hepatitis. Aliment Pharmacol Ther 2011; 33: 1273-1291.

182. Lohse AW, zum Büschenfelde KH, Franz B, Kanzler S, Gerken G, Dienes HP. Characterization of the overlap syndrome of primary biliary cirrhosis $(\mathrm{PBC})$ and autoimmune hepatitis: evidence for it being a hepatitic formof $\mathrm{PBC}$ in genetically susceptible individuals. Hepatology 1999;29:1078-1084.

183. Yoshioka Y, Taniai M, Hashimoto E, Haruta I, Shiratori K. Clinical profile of primary biliary cirrhosis with features of autoimune hepatitis: Importance of corticosteroid therapy. Hepatol Res 2014;44:947-55.

184. Chazouillères O,Wendum D, Serfaty L, Rosmorduc O, Poupon R. Long term outcome and response to therapy of primary biliary cirrhosis-autoimmune hepatitis overlap syndrome. J Hepatol 2006;44:400-406.

185. Joshi S, Cauch-Dudek K, Wanless IR, et al. Primary biliary cirrhosis with additional features of autoimmune hepatitis: response to therapy with ursodeoxycholic acid. Hepatology 2002;35(2):409-413.

186. Zhang Y, Lu J, Dai W, Wang F, Shen M, Yang J, Zhu R, Zhang H, Chen K, Cheng $\mathrm{P}, \mathrm{He}$ L, Wang C, Xu L, Zhou Y, Guo C. Combination therapy of ursodeoxycholic Acid and corticosteroids for primary biliary cirrhosis with features of autoimmune hepatitis: a meta-analysis. Gastroenterol Res Pract. 2013;2013:490731.

187. van Buuren HR, van Hoogstraten HJE, Terkivatan T, Schalm SW, Vleggaar FP. High prevalence of autoimmune hepatitis among patients with primary sclerosing cholangitis. J Hepatol 2000;33:543-548.

188. Floreani A, Rizzotto ER, Ferrara F, Carderi I, Caroli D, Blasone L, Baldo V. Clinical course and outcome of autoimmune hepatitis/primary sclerosing cholangitis overlap syndrome. Am J Gastroenterol 2005; 100: 1516-22.

189. Olsson R1, Glaumann H, Almer S, Broomé U, Lebrun B, Bergquist A, Björnsson E, Prytz H, Danielsson A, Lindgren S. High prevalence of small duct primary sclerosing cholangitis among patients with overlapping autoimmune hepatitis and primary sclerosing cholangitis. Eur J Intern Med 2009;20:190-196.

190. Lüth S, Kanzler S, Frenzel C, Kasper HU, Dienes HP, Schramm C, et al. Characteristics and long-term prognosis of the autoimmune hepatitis/primary sclerosing cholangitis overlap syndrome. J Clin Gastroenterol 2009;43:75-80.

191. Kremer AE, Elferink RPJO, Beuers U. Pathophysiology and current management of pruritus in liver diseases. Clinics and Research in Hepatology and Gastroenterology; 35:89-97, 2011.

192. Beuers U, Kremer AE, Bolier R, Elferink RPJO. Pruritus in cholestasis: facts and fiction. Hepatology; 60:399-407, 2014

193. Kremer AE, Bolier R, van Dijk R, Elferink RPJO. Advances in pathogeneisis and management of pruritus in cholestasis. Digestive Diseases; 32:637-45, 2014

194. Welter E, Bonfa R, Petry V, Moreira LL, Weber MB. Relationship between pruritus and quality of life in patients on hemodialysis. An Bras Dermatol; $83: 137-40,2008$

195. Elman S, Hynan LS, Gabriel V, Mayo MJ. The 5-D itch scale: a new measure of pruritus. Br J Dermatol; 162 (3):587-593, 2010.

196. Karlsen TH, Boberg KM. Update on primary sclerosing cholangitis. Journal of Hepatology; 59:571-582, 2013. 
197. Goldblatt J, Taylor PJ, Lipman T, Prince MI, Baragiotta A, Bassendine MF, et al. The true impact of fatigue in primary biliary cirrhosis: a population study. Gastroenterology 2002;122:1235-1241

198. Poupon RE, Chretien Y, Chazouilleres O, Poupon R, Chwalow J. Quality of life in patients with primary biliary cirrhosis. Hepatology 2004; 40:489-94.

199. Jones DE, Bala N, Burt J, Goldblatt BJ, Prince M, Newton JL. Four year follow up of fatigue in a geographically defined primary biliary cirrhosis cohort. Gut 2006; 55:536-46.

200. van Os E, van den Broek WW, Mulcer PGH, ter Borg PC, Bruijn JA, van Buuren HR. Depression in patients with primary biliary cirrhosis and primary sclerosing cholangitis. J Hepatol 2007; 46:1099-103.

201. Elta GH, Sepersky RA, Goldberg MJ, Connors CM, Miller KB, Kaplan MM. Increased incidence of hypothyroidism in primary biliary cirrhosis. Dig Dis Sci $1983 ; 28: 971-5$

202. Ian Gan S, de Jongh M, Kaplan MM. Modafinil in the treatment of debilitating fatigue in primary biliary cirrhosis: a clinical experience. Dig Dis Sci 2009; 54: 2242-6

203. Carbone M, Bufton S, Monaco A, Griffiths L, Jones DE, Neuberger JM. The effect of liver transplantation on fatigue in patients with primary biliary cirrhosis: a prospective study. J Hepatol 2013; 59: 490-

204. Longo M, Crosignani A, Battezzati PM, et al. Hyperlipidaemic state and cardiovascular risk in primary biliary cirrhosis. Gut 2002; 51: 265-9.

205. Lewis JH, Mortensen ME, Zweig S, et al. Efficacy and safety of high-dose pravastatin in hypercholesterolemic patients with well-compensated chronic liver disease: results of a prospective, randomized, double-blind, placebo-controlled, multicenter trial. Hepatology 2007; 46: 1453-63.

206. Cash WJ, O'Neill S, O'Donnell ME, McCance DR, Young IS, McEneny J, McDougall NI, Callender ME. Randomized controlled trial assessing the effect of simvastatin in primary biliary cirrhosis. Liver Inter. 2013; 33: 1166-74.

207. Guañabens N, Parés A. Management of osteoporosis in liver disease. Clin Res Hepatol Gastroenterol 2011; 35: 438-455.

208. Guichelaar M, Kendall R, Malinchoc M, Hey HE. Bone mineral density before and after OLT: Long-term follow-up and predictive factors. Liver Transpl. 2006 12:1390-1402.

209. Guichelaar M, Schmoll J, Malinchoc M, Hay JE. Fractures and avascular necrosis before and after orthotopic liver transplantation: Long-term follow-up and predictive factors. Hepatology 2007;46:1198-1207.

210. Leslie WD, Bernstein C, Leboff MS. AGA Technical review on osteoporosis in hepatic disorders. Gastroenterology 2003;125:941-966.

211. Guañabens N, Monegal A, Cerdá D, Muxí A, Gifre L, Peris P, Parés A. Randomized trial comparing monthly ibandronate and weekly alendronate for osteoporosis in patients with primary biliary cirrhosis. Hepatology 2013;58:2070-278.

212. Crawford B, Kam C, Pavlovic J, Byth K, Handelsman D, Angus P, et al. Zoledronic acid prevents bone loss after liver transplantation: a randomized double-blind, placebo-controlled trial. Ann Inter Med 2006;144:239-2

213. Misof B, Bodingbauer M, Roschger P, Wekerle T, Pakrah B, Hass M, et al. Short term effects of high dose zoledronic acid treatment on bone mineralization density distribution after orthotopiv liver transplantiom. Calcif Tissue Int 2008;83:167-175.

214. Wagner D, Amrein K, Dimai H, Kniepeiss D, Tscheliessnigg K, Kornprat P, et al Ibandronate and calcitriol reduces fracture risk, reverses bone loss, and normalizes bone turnover after ITX. Transplantation 2012;93:331-336.

215. Kaemmerer D, Schimidt B, Lehmann G, Wolf G, Hommann M, Settmacher U. Monthly ibandronate for the prevention of bone loss in patients after liver transplantation. Transplant Proc 2012;44:1362-1367.

216. Lam SK, Wong KP, Chan PK, Ngan H, Ong GB. Recurrent pyogenic cholangitis: a study by endoscopic retrograde cholangiography. Gastroenterology 1978;74:1196-1203.

217. Sans M, Rimola A, Navasa M, Grande L, Garcia-Valdecasas JC, Andreu H, Salmeron JM, et al. Liver transplantation in patients with Caroli's disease and recurrent cholangitis. Transpl Int 1997;10:241-244.

218. Gores GJ, Gish RG, Shrestha R, Wiesner RH. Model for end-stage liver disease (MELD) exception for bacterial cholangitis. Liver Transpl 2006;12:S91-92.

219. Francoz C, Belghiti J, Castaing D, Chazouillères O, Duclos-Valle JC, Duvoux C, Lerut J, Le Treut YP, Moureau R, Mandot A, Pageaux G, Samuel D, Thabut D, Valla D, Durand F. Model for End-Stage Liver Disease Exceptions in the Context of the French Model for End-Stage Liver Disease Score-Based Liver Allocation System. Liver Transpl 2011;17:1137-1151.

220. Wiesner RH, LaRusso NF. Clinicopathologic features of the syndrome of primary sclerosing cholangitis. Gastroenterology 1980;79:200-206.

221. Goldberg DS, Camp A, Martinez-Camacho A, Forman L, Fortune B, Reddy KR. Risk of waitlist mortality in patients with primary sclerosing cholangitis and bacterial cholangitis. Liver Transpl 2013;19:250-258.
222. Freeman RB, Jr., Gish RG, Harper A, Davis GL, Vierling J, Lieblein L, Klintmalm $\mathrm{G}$, et al. Model for end-stage liver disease (MELD) exception guidelines: results and recommendations from the MELD Exception Study Group and Conference (MESSAGE) for the approval of patients who need liver transplantation with diseases not considered by the standard MELD formula. Liver Transpl 2006;12:S128-136

223. Bilbao MK, Dotter CT, Lee TG, Katon RM. Complications of endoscopic retrograde cholangiopancreatography (ERCP). A study of 10,000 cases. Gastroenterology 1976;70:314-320.

224. Tabibian JH, Weeding E, Jorgensen RA, Petz JL, Keach JC, Talwalkar JA, Lindor KD. Randomised clinical trial: vancomycin or metronidazole in patients with primary sclerosing cholangitis - a pilot study. Aliment Pharmacol Ther 2013;37:604-612.

225. Cotton PB, Connor P, Rawls E, Romagnuolo J. Infection after ERCP, and antibiotic prophylaxis: a sequential quality-improvement approach over 11 years. Gastrointest Endosc 2008;67:471-475.

226. Banerjee S, Shen B, Baron TH, Nelson DB, Anderson MA, Cash BD, Dominitz JA, et al. Antibiotic prophylaxis for GI endoscopy. Gastrointest Endosc 2008;67:791-798.

227. Mani V, Cartwright K, Dooley J, Swarbrick E, Fairclough P, Oakley C. Antibiotic prophylaxis in gastrointestinal endoscopy: a report by a Working Party for the British Society of Gastroenterology Endoscopy Committee. Endoscopy 1997;29:114-119.

228. Antibiotic prophylaxis for gastrointestinal endoscopy. American Society for Gastrointestinal Endoscopy. Gastrointest Endosc 1995;42:630-635.

229. Pohl J, Ring A, Stremmel W, Stiehl A. The role of dominant stenoses in bacterial infections of bile ducts in primary sclerosing cholangitis. Eur J Gastroenterol Hepatol 2006;18:69-74

230. Tabibian JH, Lindor KD. Primary sclerosing cholangitis: a review and update on therapeutic developments. Expert Rev Gastroenterol Hepatol 2013;7:103-114

231. Elfaki DA, Lindor KD. Antibiotics for the treatment of primary sclerosing cholangitis. Am J Ther 2011;18:261-265.

232. Farkkila M, Karvonen AL, Nurmi H, Nuutinen H, Taavitsainen M, Pikkarainen P, Karkkainen P. Metronidazole and ursodeoxycholic acid for primary sclerosing cholangitis: a randomized placebo-controlled trial. Hepatology 2004;40:1379-1386.

233. Chapman MH, Webster GJ, Bannoo S, Johnson GJ, Wittmann J, Pereira SP. Cholangiocarcinoma and dominant strictures in patients with primary sclerosing cholangitis: a 25-year single-centre experience. Eur J Gastroenterol Hepatol 2012;24:1051-1058.

234. Ahrendt SA, Pitt HA, Kalloo AN, Venbrux AC, Klein AS, Herlong HF, Coleman J, et al. Primary sclerosing cholangitis: resect, dilate, or transplant? Ann Surg 1998;227:412-423.

235. Myburgh JA. Surgical biliary drainage in primary sclerosing cholangitis. The role of the Hepp-Couinaud approach. Arch Surg 1994;129:1057-1062.

236. Cameron JL, Pitt HA, Zinner MJ, Herlong HF, Kaufman SL, Boitnott JK, Coleman J. Resection of hepatic duct bifurcation and transhepatic stenting for sclerosing cholangitis. Ann Surg 1988;207:614-622.

237. Pawlik TM, Olbrecht VA, Pitt HA, Gleisner AL, Choti MA, Schulick RD, Cameron JL. Primary sclerosing cholangitis: role of extrahepatic biliary resection. J Am Coll Surg 2008;206:822-830; discussion 830-822.

238. Claessen MMH, Vleggaar FP, Tytgat KMAJ, Siersema PD, van Buuren HR High lifetime risk of cancer in primary sclerosing cholangitis. J Hepatol. 2009 Jan;50(1):158-64.

239. Boberg KM, Bergquist A, Mitchell S, Pares A, Rosina F, Broomé U, et al Cholangiocarcinoma in primary sclerosing cholangitis: risk factors and clinical presentation. Scand J Gastroenterol. 2002 Oct;37(10):1205-11.

240. Bergquist A, Ekbom A, Olsson R, Kornfeldt D, Lööf L, Danielsson A, et al. Hepatic and extrahepatic malignancies in primary sclerosing cholangitis. $\mathrm{J}$ Hepatol. 2002 Mar;36(3):321-7

241. Wiencke K, Boberg KM. Current consensus on the management of primary sclerosing cholangitis. Clin Res Hepatol Gastroenterol. 2011 Dec;35(12):786-91.

242. Razumilava N, Gores GJ. Cholangiocarcinoma. The Lancet. 2014 Jun;383(9935):2168-79.

243. Burak K, Angulo P, Pasha TM, Egan K, Petz J, Lindor KD. Incidence and risk factors for cholangiocarcinoma in primary sclerosing cholangitis. Am J Gastroenterol. 2004 Mar;99(3):523-6.

244. Razumilava N, Gores GJ, Lindor KD. Cancer surveillance in patients with primary sclerosing cholangitis. Hepatology. 2011 Nov;54(5):1842-52.

245. Charatcharoenwitthaya P, Enders FB, Halling KC, Lindor KD. Utility of serum tumor markers, imaging, and biliary cytology for detecting cholangiocarcinoma in primary sclerosing cholangitis. Hepatology. 2008 Oct;48(4):1106-17.

246. Levy C, Lymp J, Angulo P, Gores GJ, Larusso N, Lindor KD. The value of serum CA 19-9 in predicting cholangiocarcinomas in patients with primary sclerosing cholangitis. Dig Dis Sci. 2005 Sep;50(9):1734- 40.

247. Said K, Glaumann H, Bergquist A. Gallbladder disease in patients with primary sclerosing cholangitis. J Hepatol. 2008 Apr;48(4):598-605. 
248. Neuberger JM, Carbone M. Autoimmune liver disease, autoimmunity and liver transplantation. J Hepatol. 2014 Jan;60(1):210-23.

249. Karlsen TH, Vesterhus M, Boberg KM. Review article: controversies in the management of primary biliary cirrhosis and primary sclerosing cholangitis. Aliment Pharmacol Ther. 2014;39(3):282-301.

250. Invernizzi P, Mackay IR. Transplantation in autoimmune liver diseases. World J Gastroenterol 2008 June 7; 14(21): 3388-3395.

251. Genda T, Ichida T, Shotaro S, Sata M, Tanaka E, Inui A, Egawa H, Umeshita K, Furukawa H, Kawasaki S, Inomata Y. Waiting list mortality of patients with primary biliary cirrhosis in the Japanese transplant allocation system. J Hepato 2014; 49:324-31.

252. Lammers WJ, Kowdley KV, Buuren HR. Predicting outcome in primary biliary cirrhosis. Ann Hepatol 2014; 13:316-26.

253. Gitto S, Lorenzini S, Biselli M, Conti F, Andreoni P, Bernardi M. Allocation priority in non-urgent liver transplantation: An overview of proposed scoring systems. Digestive and Liver Disease 41 (2009) 700-706.
254. Weinmann A, Sattler T, Unold HP, Grambihler A, Teufel A, Koch S, Schuchmann M, Biesterfeld S, Wörns MA, Galle PR, Schulze-Bergkamen H. Predictive Scores in Primary Biliary Cirrhosis: A Retrospective Single Center Analysis of 204 Patients. J Clin Gastroenterol. 2015;49:438-47.

255. Yeoman, AD, Westbrook, RH, Zen Y, Bernal W, Al-Chalabi T, Wendon JA, O'Grady JG, Heneghan MA. Prognosis of acute severe autoimmune hepatitis (AS-AIH): The role of corticosteroids in modifying outcome. J Hepatol, 2014; 61:876-82

256. Weiler-Normann C, Lohse. Acute autoimmune hepatitis: Many open questions. Editorial. J Hepatol 2014; 61:727-29.

257. Liberal R, Longhi MS, Grant CR, Mieli-Vergani G, Vergani D. Autoimmune hepatitis after liver transplantation. Clin Gastroenterol Hepatol 2012;10:346-353.

258. Eaton, J. E., Talwalkar, J. A., Lazaridis, K. N. et al. Pathogenesis of Primary Sclerosing Cholangitis and Advances in Diagnosis and Management. Gastroenterology, 145:521-36. 2013 